\title{
Synergistic Experimental and Computational Investigation of the Bioorthogonal Reactivity of Substituted Aryltetrazines
}

Umberto M. Battisti ${ }^{\dagger a}$, Rocío García-Vázquez ${ }^{\dagger a}$, Dennis Svatunek ${ }^{\mathrm{c}}$, Barbara Hermann ${ }^{\mathrm{c}}$, Andreas Löffler $^{\mathrm{c}}$, Hannes Mikulac ${ }^{\mathrm{c}}$, Matthias Manfred Herth*a, b

${ }^{a}$ Department of Drug Design and Pharmacology, Faculty of Health and Medical Sciences, University of Copenhagen, Jagtvej 160, 2100 Copenhagen; 'bepartment of Clinical Physiology, Nuclear Medicine \& PET, Rigshospitalet, Blegdamsvej 9, 2100 Copenhagen, Denmark; ' Institute of Applied Synthetic Chemistry, Technische Universität Wien (TU Wien), Getreidemarkt 9, 1060 Vienna, Austria; †These authors contributed equally

*Corresponding Author: matthias.herth@sund.ku.dk; Phone: +45 35336624

${ }^{\dagger}$ These authors contributed equally to the work

Key words: Tetrazine ligation, Reaction Kinetics, Pretargeting, Rate Constants

\begin{abstract}
Tetrazines $(\mathrm{Tz})$ have been applied as bioorthogonal agents for various biomedical applications including pretargeted imaging approaches. In radioimmunoimaging, pretargeting increases the targetto-background ratio while simultaneously reducing the radiation burden. We have recently reported a strategy to directly ${ }^{18} \mathrm{~F}$-label highly reactive tetrazines based on a 3-(3-fluorophenyl)-Tz core structure. Herein, we report a kinetic study on this versatile scaffold. A library of 40 different tetrazines was prepared, fully characterized, and investigated with emphasis on second order rate constants for the reaction with trans-cyclooctene (TCO). Our results reveal the effects of various substitution patterns and moreover demonstrate the importance of measuring reactivities in the solvent of interest, as click rates in different solvents do not necessarily correlate well. In particular, we report that tetrazines modified in 2-position of the phenyl substituent show high intrinsic reactivity towards TCO, which is diminished in aqueous systems by unfavorable solvent effects. The obtained results enable the prediction of the bioorthogonal reactivity and thereby facilitate the development of the next-generation of substituted aryltetrazines for in vivo application.
\end{abstract}




\section{Introduction}

Bioorthogonal reactions are molecular transformations not present in nature. ${ }^{[1]}$ The term was introduced by Bertozzi and refers to a chemical reaction that does not interact or interfere with biological systems and functionalities. ${ }^{[2]}$ In recent years, bioorthogonal reactions have become increasingly popular, enabling a number of applications in various fields. ${ }^{[1,3]}$ The common features of these transformations include fast and efficient reaction at low concentrations in biological media at physiological $\mathrm{pH}$, high selectivity and no cross-reactivity with functional groups present in biomolecules, and the formation of stable ligation products. ${ }^{[1,3]}$ Bioorthogonal reactions have attracted increasing interest in studying biological processes due to their unique properties. ${ }^{[3-4]}$ Amongst all bioorthogonal reactions reported so far, the [4+2] cycloaddition of 1,2,4,5-tetrazines (Tz) and trans-cyclooctenes (TCOs) stands out due to exceptional reaction kinetics (Figure 1). ${ }^{[5]}$ This inverse electron-demand Diels-Alder (IEDDA)-initiated bioorthogonal reaction was shown to be highly selective and compatible with biological systems, even enabling its application in vivo. ${ }^{[5 b]}$ In recent years, tetrazine ligations have been exploited in biomedical research, in particular for rapid bioconjugation, drug delivery, molecular imaging, drug-target identification, and radiochemistry. ${ }^{[6]}$ Tetrazine ligations have been extensively used in pretargeted positron emission tomography (PET) imaging in which high reaction rate constants are essential due to the very low concentrations of the radiolabeled compounds in vivo. ${ }^{[6]}$ Several preclinical examples exist demonstrating the potential of this approach. ${ }^{[7]}$

A)

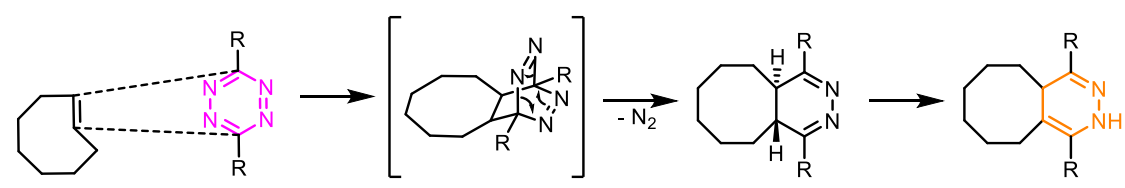

B)

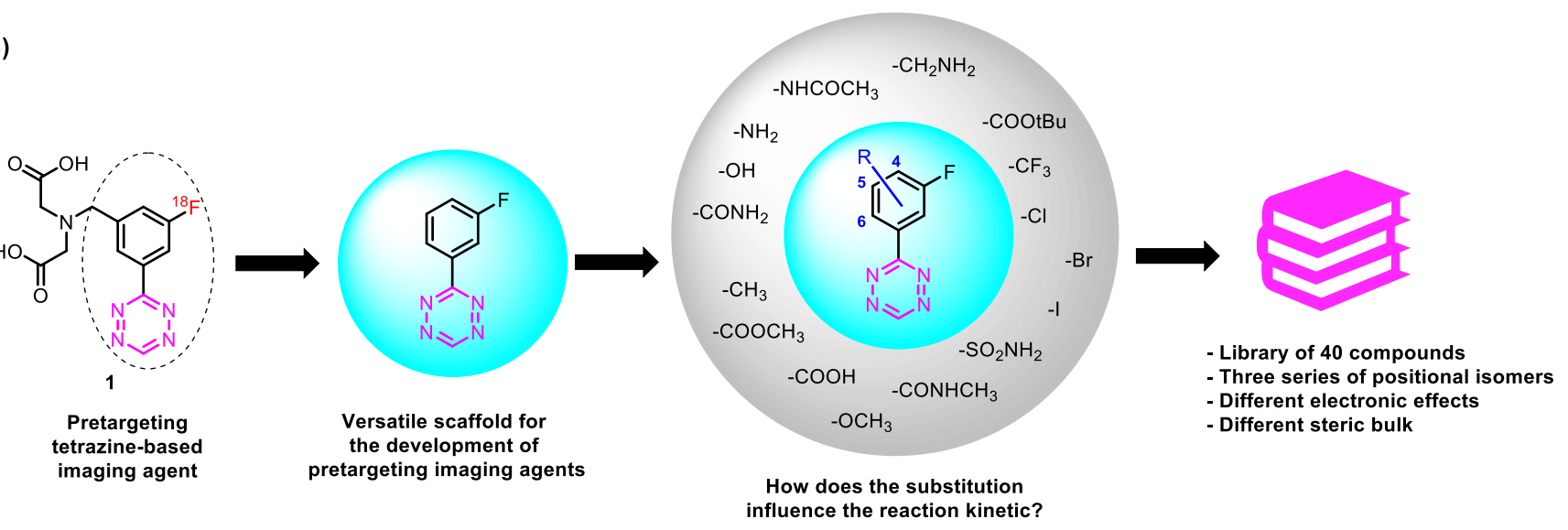

Figure 1. A) Tetrazine ligation mechanism. B) Compound $\mathbf{1}$ and the different substituents employed for the structurekinetics relationship on the 3-(3-fluorophenyl)-1,2,4,5-tetrazine core. 
Since fluorine-18 $\left({ }^{18} \mathrm{~F}\right)$ possesses almost ideal physical characteristics for molecular imaging, an ${ }^{18} \mathrm{~F}$ labeled Tz would be ideal for PET applications in a clinical setting. ${ }^{[8]}$ However, due to the intrinsic instability of the tetrazine core to basic conditions typically employed in direct ${ }^{18} \mathrm{~F}$-fluorination approaches, no ${ }^{18} \mathrm{~F}$-tetrazine was synthesized until a few years ago. ${ }^{[9]}$ Recently, we reported the first direct ${ }^{18} \mathrm{~F}$-labeling of highly reactive tetrazines, applying copper-mediated oxidative ${ }^{18} \mathrm{~F}$ fluorination. ${ }^{[10]}$ This method allowed us to develop a hydrophilic, fast-clearing, and highly reactive bioorthogonal click imaging agent (1, Figure 1B). ${ }^{[10]} 3$-(3-Fluorophenyl)-1,2,4,5-tetrazine, the scaffold of compound $\mathbf{1}$, could be further modified with different moieties to obtain pretargeting agents with peculiar physicochemical parameters. To enable the rational design of tetrazines, it is however important to understand the relationship of the substitution pattern of Tz-derivatives and their reactivity towards TCOs, which motivated this study (Figure 1). In general, it is well-known that electron-withdrawing substituents increase, and electron-donating groups decrease the reactivity. ${ }^{[11]}$ Nevertheless, only few systematic studies of the effect of various substituents of the phenyl ring of $\mathrm{Tz}$ derivatives on reaction kinetics have been reported so far, while most of them focus on the substituents on the Tz itself. ${ }^{[5 b]}$ Consequently, we prepared a library of $40 \mathrm{Tz}$ derivatives with a set of 16 different phenyl-substituents and evaluated the reaction kinetics of the ligation with TCO both in Dulbecco's phosphate buffered saline (DPBS) and acetonitrile $\left(\mathrm{CH}_{3} \mathrm{CN}\right)$. Based on the obtained data, we developed a method to predict the IEDDA reactivity of differently substituted Tzs.

\section{Result and Discussion}

\section{Library Design}

Sixteen different moieties were chosen to study the effect of different substituents on the reactivity of the 3-(3-fluorophenyl)-1,2,4,5-tetrazine core (Figure 1), covering a broad set of electronwithdrawing, electron-donating, and sterically demanding groups. In addition, 4-, 5- and 6-modified isomers were included in the library to study effects of the substitution pattern.

\section{Synthesis}

For the synthesis of tetrazines 2a-c, 3a-c and 5-10a-c, the required nitriles were commercially available. Boc-protected nitriles for the synthesis of Tzs 4a-c were obtained by reacting the corresponding bromobenzyl compounds with phthalimide potassium salt, followed by hydrazine deprotection. ${ }^{[12]}$ The primary amines were then reacted with di-tert-butyl dicarbonate to afford the protected compounds in good overall yields (Scheme 1). 


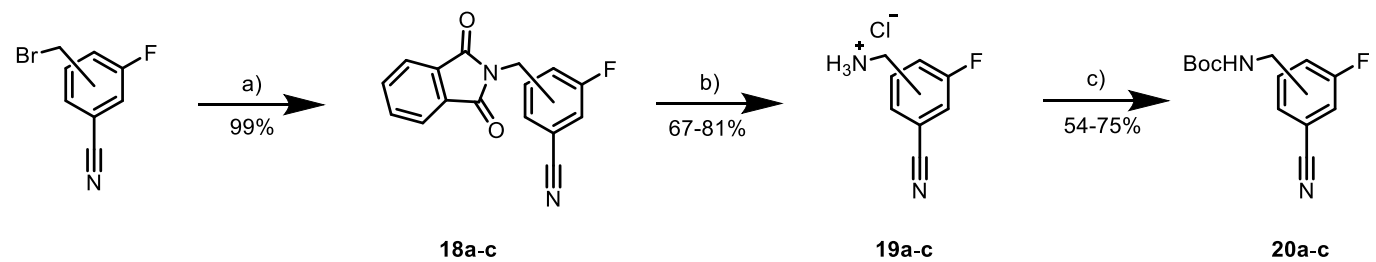

Scheme 1. Synthesis of (aminomethyl)benzonitrile derivatives - a) Phthalimide potassium salt, DMF, $9 \mathrm{~h}, 130^{\circ} \mathrm{C}$; b) $i$ ) $\mathrm{NH}_{2} \mathrm{~N}_{2} \cdot \mathrm{H}_{2} \mathrm{O}$, EtOH, reflux, 2h; ii) $\mathrm{HCl}, \mathrm{Et}_{2} \mathrm{O}, 0{ }^{\circ} \mathrm{C}, 1 \mathrm{~h}$; c) $\mathrm{Boc}_{2} \mathrm{O}, \mathrm{Et}_{3} \mathrm{~N}, \mathrm{CH}_{3} \mathrm{CN}, \mathrm{rt}, 12 \mathrm{~h}$.

The tert-butyl ester nitriles for the synthesis of Tzs 12a-c were obtained from the corresponding carboxylic acids (Scheme 2A). ${ }^{[13]}$ Similarly, the primary amides for Tzs 13a-c and the secondary amides for Tzs 14a-c were obtained in yields ranging from 76 to $80 \%$ by CDI-mediated activation of the carboxyl group and reaction with ammonia and methylamine, respectively (Scheme 2A). Bocprotection and acylation of the corresponding aniline yielded the nitriles as starting materials for the synthesis of Tzs 15a-c and Tzs 16a-c (Scheme 2B). Finally, the sulfonamides Tzs 17a-c were prepared using a modified Sandmeyer procedure followed by reaction with ammonia (Scheme 2B). ${ }^{[14]}$

A)

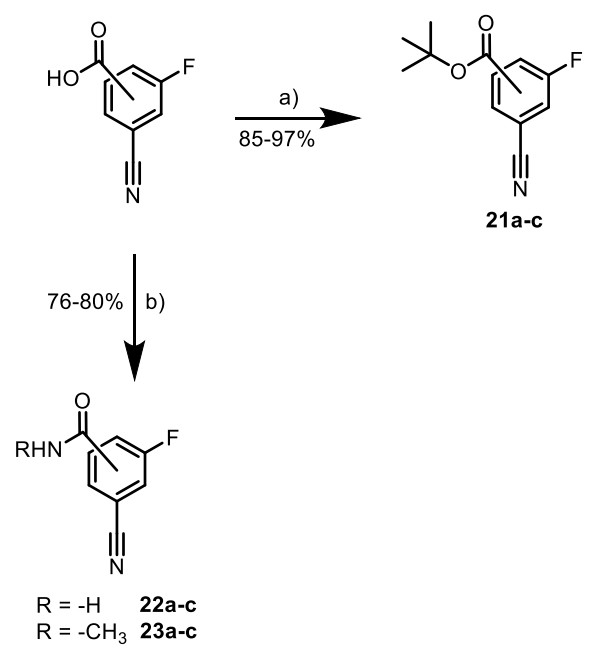

B)

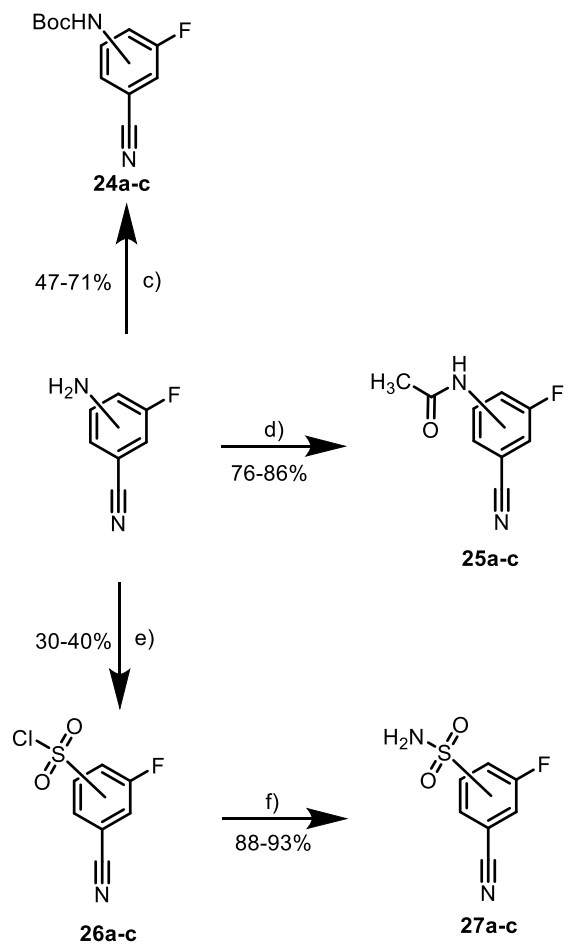

Scheme 2. A) Synthesis of tert-butyl ester and amide derivatives. B) Synthesis of Boc-protected aniline, acetamide and sulfonamide derivatives. - a) $t \mathrm{BuOH}$, DMAP, BOC $2 \mathrm{O}$, THF, rt, $12 \mathrm{~h}$; b) $\mathrm{NH}_{3}\left(35 \%\right.$ in $\left.\mathrm{H}_{2} \mathrm{O}\right)$ or $\mathrm{CH}_{3} \mathrm{NH}_{2}\left(40 \%\right.$ in $\left.\mathrm{H}_{2} \mathrm{O}\right)$, $\mathrm{CDI}, \mathrm{CH}_{3} \mathrm{CN}, \mathrm{rt}, 2 \mathrm{~h}$; c) $\mathrm{Boc}_{2} \mathrm{O}, \mathrm{Et}_{3} \mathrm{~N}, \mathrm{CH}_{2} \mathrm{Cl}_{2}, \mathrm{rt}, 12 \mathrm{~h}$; d) $\mathrm{Ac}_{2} \mathrm{O}, \mathrm{CH}_{2} \mathrm{Cl}_{2}, \mathrm{rt}, 24 \mathrm{~h}$; e) i) $\mathrm{HCl}, \mathrm{NaNO}_{2}, 0^{\circ} \mathrm{C}$ to rt, 2 h; ii) $\mathrm{SO}_{2}, \mathrm{CuCl} \mathrm{AcOH}, 0{ }^{\circ} \mathrm{C}$ to rt, $\left.1 \mathrm{~h} ; \mathbf{f}\right) \mathrm{NH}_{3}, \mathrm{MeOH}, \mathrm{CH}_{3} \mathrm{CN}, 0{ }^{\circ} \mathrm{C}$ to rt, $2 \mathrm{~h}$.

Tetrazines were synthesized using a metal-free synthetic approach reported by Qu et al. (Scheme 3). ${ }^{[15]}$ 4- and 5-substituted derivatives were isolated in modest yields using this procedure. 6substituted derivatives were more difficult to prepare. Only $\mathbf{2 c}$ and $\mathbf{4 c - 9 c}$ could be obtained suggesting that electron withdrawing or bulky groups at the 6-position hinder the reaction or generate highly 
unstable tetrazines. Deprotection of Tzs 28a-c, 29a, and 29b resulted in Tzs 4a-c, 15a, and 15b in yields ranging from 46 to $97 \%$. Methyl esters 11a and 11b were prepared by acid-catalyzed esterification of the corresponding carboxylic acids $\mathbf{1 0 a}$ and $\mathbf{1 0 b}$ respectively in 85 and $66 \%$ yields.

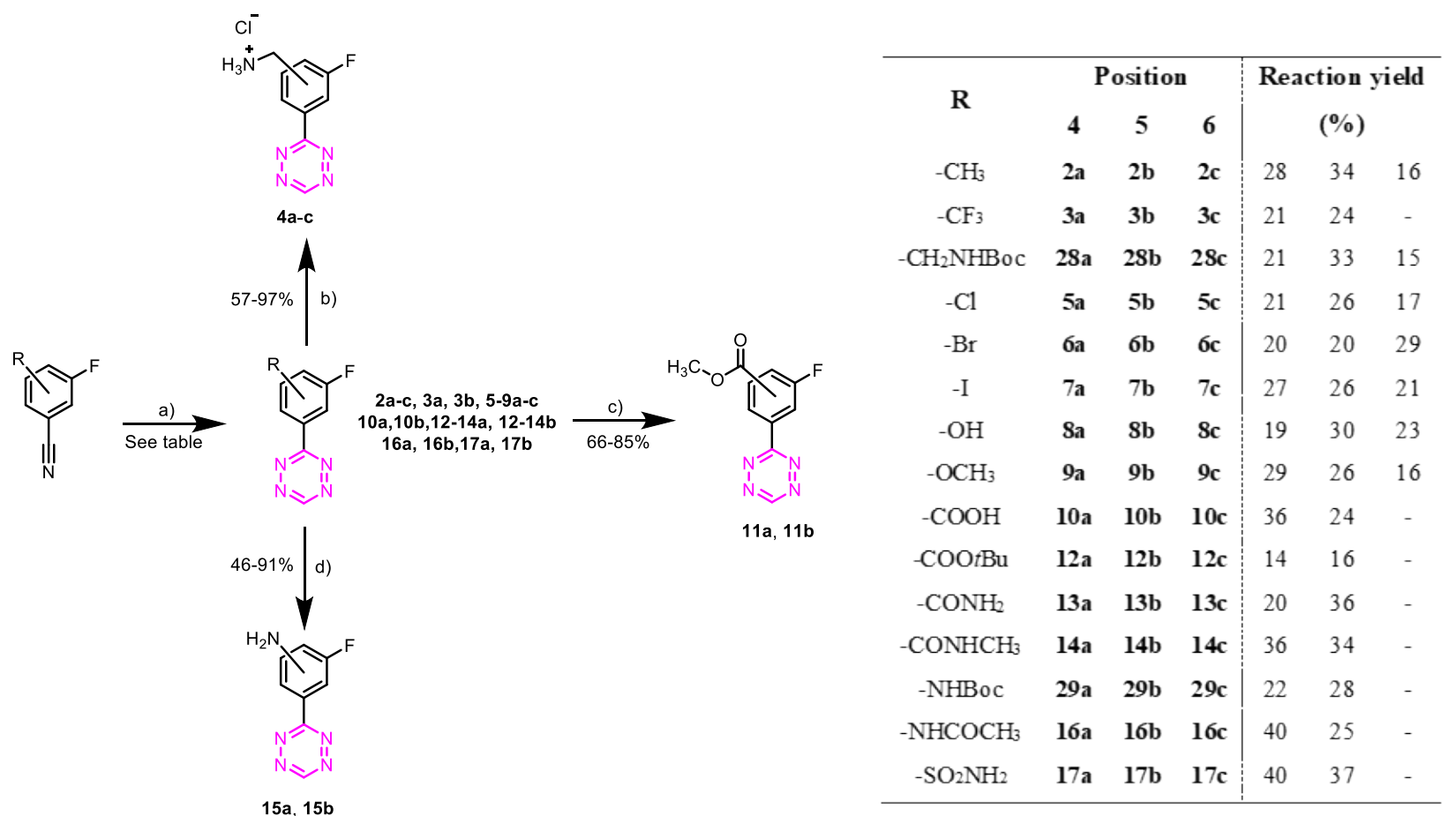

Scheme 3. Synthesis of 3-(3-fluorophenyl)-1,2,4,5-tetrazine derivatives - a) i) $\mathrm{NH}_{2} \mathrm{NH}_{2} \cdot \mathrm{H}_{2} \mathrm{O}, \mathrm{CH}_{2} \mathrm{Cl}_{2}, \mathrm{~S} 8, \mathrm{EtOH}, 50{ }^{\circ} \mathrm{C}$, $24 \mathrm{~h}$; ii) $\mathrm{NaNO}_{2}, \mathrm{AcOH}, 0^{\circ} \mathrm{C}$ to rt, $30 \mathrm{~min}$; b) $\mathrm{HCl}$, dioxane, $\mathrm{CH}_{2} \mathrm{Cl}_{2}$, rt, $2 \mathrm{~h}$; c) $\mathrm{HCl}$, dioxane, $\mathrm{MeOH}, \mathrm{rt}, 3 \mathrm{~h}$; d) TFA, $\mathrm{CH}_{2} \mathrm{Cl}_{2}, \mathrm{rt}, 2 \mathrm{~h}$.

\section{Reaction kinetics}

Reactivities of the tetrazines in the reaction with TCO were determined by pseudo-first order measurements in $\mathrm{CH}_{3} \mathrm{CN}$ at $25^{\circ} \mathrm{C}$ and in DPBS at $37{ }^{\circ} \mathrm{C}$ by stopped-flow spectrophotometry. ${ }^{[16]}$ Solutions of TCO in anhydrous $\mathrm{CH}_{3} \mathrm{CN}$ and axTCO-PEG 4 in DPBS were employed for the analysis. ${ }^{[16-17]}$ The data obtained are reported in Table 1. 
Table 1. Second-order rate constants for the reaction of tetrazines with trans-cyclooctenes determined by stopped-flow spectrophotometry.

\begin{tabular}{|c|c|c|c|c|c|c|c|c|c|c|}
\hline \multicolumn{2}{|c|}{ Tetrazine } & \multicolumn{3}{|c|}{$\begin{array}{c}\text { Compound } \\
\text { Number }\end{array}$} & \multicolumn{3}{|c|}{$\begin{array}{c}\text { Rate constant }\left(M^{-1} \mathbf{s}^{-1}\right) \\
\text { in DPBS }\end{array}$} & \multicolumn{3}{|c|}{$\begin{array}{c}\text { Rate constant }\left(\mathrm{M}^{-1} \mathrm{~s}^{-1}\right) \\
\qquad \text { in } \mathrm{CH}_{3} \mathrm{CN}\end{array}$} \\
\hline \multirow{2}{*}{ Structure } & \multirow{2}{*}{$\begin{array}{c}\text { Substituent } \\
\text { (R) }\end{array}$} & \multicolumn{3}{|c|}{ Position } & \multicolumn{3}{|c|}{ Position } & \multicolumn{3}{|c|}{ Position } \\
\hline & & 4 & 5 & 6 & 4 & 5 & 6 & 4 & 5 & 6 \\
\hline & $-\mathrm{CH}_{3}$ & $2 a$ & $2 b$ & $2 c$ & 68200 & 78800 & 55100 & 890 & 1060 & $-{ }^{d}$ \\
\hline & $-\mathrm{CF}_{3}$ & $3 a$ & $3 b$ & $3 c$ & 123000 & 99600 & ${ }^{\mathrm{a}}$ & 2680 & 2200 & $-{ }^{\mathrm{a}}$ \\
\hline & $-\mathrm{CH}_{2} \mathrm{NH}_{2}$ & $4 a$ & $4 b$ & $4 c$ & 74800 & 73000 & $-c$ & 4180 & $-{ }^{d}$ & 1710 \\
\hline & $-\mathrm{Cl}$ & $5 a$ & $5 b$ & $5 c$ & 90800 & 108000 & 41400 & 1650 & 2100 & 678 \\
\hline & $-\mathrm{Br}$ & $6 a$ & $6 b$ & $6 c$ & 85260 & 110000 & 33200 & 1540 & 2090 & 521 \\
\hline & $-\mathrm{I}$ & $7 a$ & $7 b$ & $7 c$ & 100000 & 104000 & 25200 & 1680 & 2040 & 403 \\
\hline & $-\mathrm{OH}$ & $8 a$ & $8 b$ & $8 c$ & 18800 & 82500 & 53500 & 531 & 1250 & 1150 \\
\hline & $-\mathrm{OCH}_{3}$ & $9 a$ & $9 b$ & $9 c$ & 48700 & 87900 & 81700 & 559 & 1290 & 1040 \\
\hline & $-\mathrm{COOH}$ & $10 a$ & $10 b$ & $10 c$ & 80800 & 90700 & $-^{\mathrm{a}}$ & 2320 & 1980 & $-{ }^{\mathrm{a}}$ \\
\hline & $-\mathrm{COOCH}_{3}$ & $11 a$ & $11 b$ & $11 c$ & 126000 & 104000 & $-^{\mathrm{a}}$ & 2340 & 1780 & $-^{\mathrm{a}}$ \\
\hline & $-\mathrm{COO} t \mathrm{Bu}$ & $12 a$ & $12 b$ & $12 c$ & 133000 & $-b$ & $-^{\mathrm{a}}$ & 2330 & 1620 & $-^{\mathrm{a}}$ \\
\hline & $-\mathrm{CONH}_{2}$ & $13 a$ & $13 b$ & $13 c$ & 111000 & 93000 & $-\mathrm{a}$ & 2010 & 1920 & $-\mathrm{a}$ \\
\hline & $-\mathrm{CONHCH}_{3}$ & $14 a$ & $14 b$ & $14 c$ & 104000 & 88500 & $-\mathrm{a}^{\mathrm{a}}$ & 1980 & 1850 & $-{ }^{\mathrm{a}}$ \\
\hline & $-\mathrm{NH}_{2}$ & $15 a$ & $15 b$ & $15 c$ & 31700 & 81400 & $-^{\mathrm{a}}$ & 274 & $-d$ & $-^{\mathrm{a}}$ \\
\hline & $-\mathrm{NHCOCH}_{3}$ & $16 a$ & $16 b$ & $16 c$ & 73900 & 99200 & $-^{\mathrm{a}}$ & 878 & 1380 & $--^{a}$ \\
\hline & $-\mathrm{SO}_{2} \mathrm{NH}_{2}$ & $17 a$ & $17 b$ & $17 c$ & 118000 & 105000 & $-{ }^{\mathrm{a}}$ & 2950 & 2710 & $-\mathrm{a}^{\mathrm{a}}$ \\
\hline
\end{tabular}

Notes: ${ }^{a}$ The corresponding Tz could not be obtained or isolated. ${ }^{b}$ The compound is not soluble in DPBS. ${ }^{\mathrm{c}}$ The compound decom-posed in DPBS. ${ }^{\mathrm{d}}$ The compound is not soluble in $\mathrm{CH}_{3} \mathrm{CN}$.

\section{Reactivity Analysis}

Measured rate constants in aqueous solution are considerably higher with accelerations of about two orders of magnitude compared to the aprotic and less polar acetonitrile. This effect has been observed before and is well known for Diels-Alder reactions between reactants that are able to form hydrogen bonds. ${ }^{[18]}$

Importantly, the rate constants measured in $\mathrm{CH}_{3} \mathrm{CN}$ show no correlation to those obtained in DPBS (Figure 2). Several of the used tetrazines change their protonation state between these two solvents. Carboxylic acids (10a and 10b) are protonated in acetonitrile but deprotonated in DPBS at pH 7.4. Similarly, phenols can be deprotonated at this $\mathrm{pH}$. We used the computational method by Shields and coworkers to estimate the $\mathrm{pKa}$ of $\mathbf{8 a - c} .{ }^{[19]}$ Our calculations gave $\mathrm{pKa}$ values for $\mathbf{8 b}$ and $\mathbf{8 c}$ of 12.2 and 14.8, respectively, indicating no deprotonation at $\mathrm{pH}$ 7.4. Tetrazine 8a is partly deprotonated in buffered solution based on a calculated $\mathrm{pKa}$ of 7.5. This deprotonation in DPBS leads to electrondonation (in contrast to the electron-withdrawing effect of the protonated carboxyl-group in acetonitrile), thus resulting in a lower reactivity in aqueous solution. Compounds $\mathbf{4 a - c}$ were prepared as $\mathrm{HCl}$ salts. Hence, $\mathbf{4 a}$ was protonated in acetonitrile, but in its neutral state at $\mathrm{pH}$ 7.4. Eliminating 
tetrazines with different protonation states from the analysis results in a linear relationship between the second order rate constants in acetonitrile and DPBS $\left(\mathrm{R}^{2}=0.83\right)$. The possibility of different protonation states and the limited correlation highlights the importance of performing kinetic measurements in aqueous and $\mathrm{pH}$-controlled solution if data is needed to estimate reactivity for bioorthogonal application in biological systems. Therefore, the following analyses are based on the values obtained in DPBS.

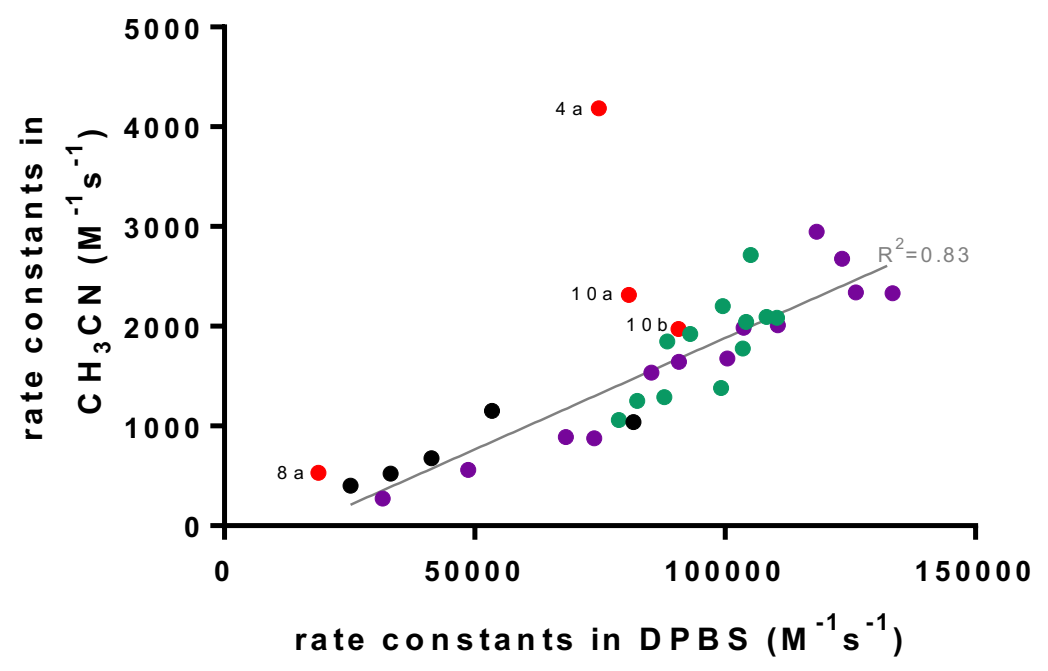

Figure 2. Correlation between second-order rate constants measured in DPBS and $\mathrm{CH}_{3} \mathrm{CN}$. Compounds are shown in purple (a-series), green (b-series), and black (c-series) with compounds depicted in red not included in the correlation due to different protonation states.

The measured second-order rate constants for the reaction of tetrazines with axTCO-PEG 4 in DPBS range from approximately 20,000 to $130,000 \mathrm{M}^{-1} \mathrm{~S}^{-1}$ for 4-substituted derivatives, while in case of 5substituted compounds the values range from 70,000 to $110,000 \mathrm{M}^{-1} \mathrm{~s}^{-1}$. 6-Substituted tetrazines show lower rates in the range of 25,000 to $80,000 \mathrm{M}^{-1} \mathrm{~s}^{-1}$. In most cases, the 5-substituted derivatives show higher reactivity than the respective 4-substituted isomer, with the 6-substituted compound being the least reactive one. A notable exception is the series 8a-c where 8a shows the lowest reactivity due to the deprotonated nature of this Tz. Most substituents are influencing the electronics of the $\pi$-system through resonance. These mesomeric effects are strongest in ortho and para position, which explains the stronger influence and higher variation in the a-series (substituent in para-position to the tetrazine) compared to compounds $\mathbf{b}$ (substituents in meta-position to the tetrazine). The reactivities of 4substituted tetrazines correlate well with Hammett $\delta_{M}$ parameters (Figure 3A, Table S2), demonstrating a mainly frontier molecular orbital (FMO) control of reactivity. ${ }^{[20]}$ The rate constants measured for 5- and 6-substituted phenyltetrazines, however, did not correlate well with Hammett 
constants (Figure 3B,C). This model only considers the influence of one substituent in isolation, hence not considering the fluorination of the phenyl-substituent.

A)

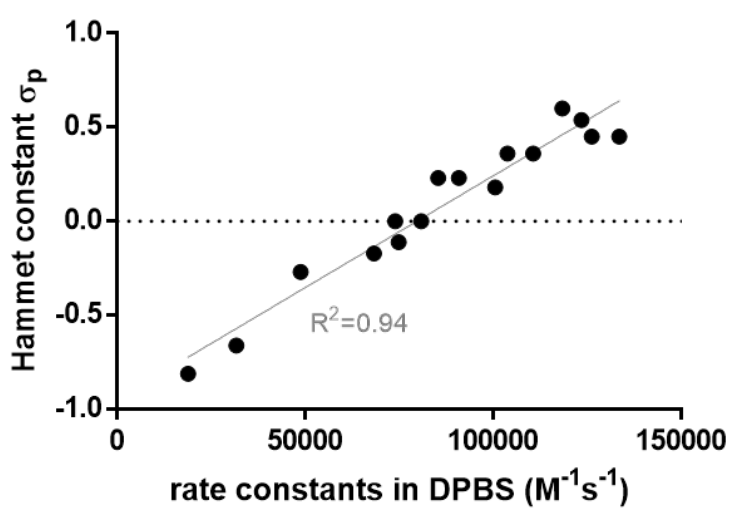

C)

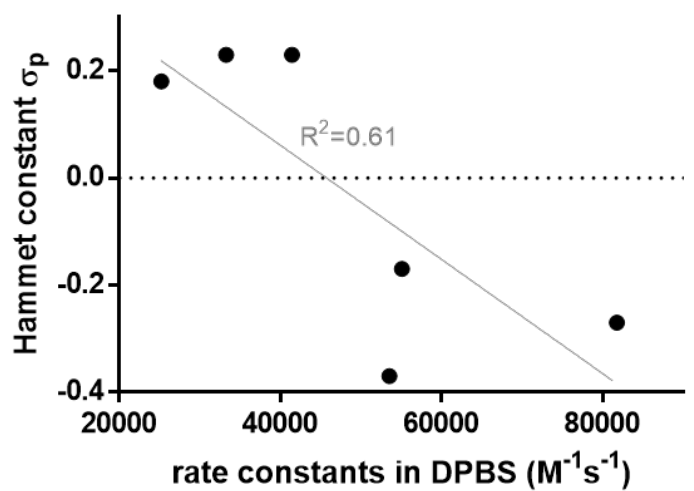

B)

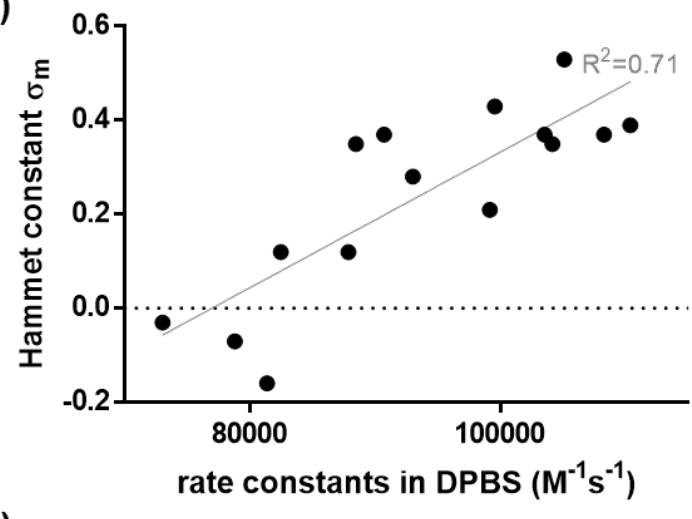

D)

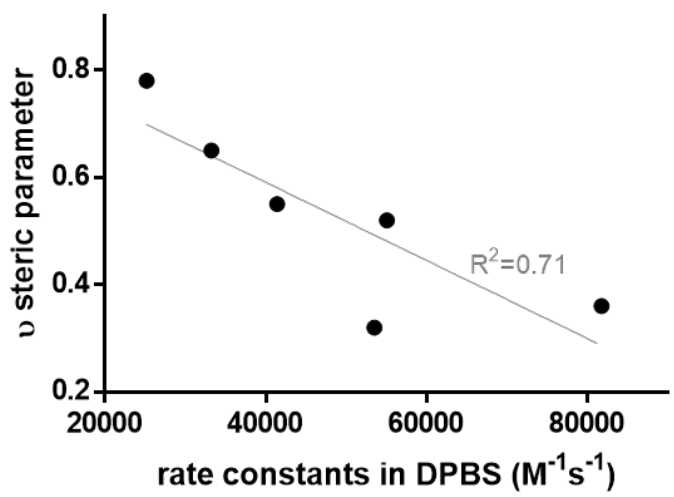

Figure 3. Correlation between measured second-order rate constants and Hammett parameters $\delta$ for A) 4-substituted, B) 5-substituted, and C) 6-substituted phenyltetrazines. D) Correlation between the steric parameter $v$ and rate constants for 6-substituted phenyltetrazines. ${ }^{[7 \mathrm{~d}, 20]}$

In the case of 6-substituted derivatives, we have focused on potential steric effects, which need to be considered. Plotting the rate constants of these tetrazines against the $v$ steric parameter shows that sterically more demanding substituents lead to lower reaction rates (Figure 3D, Table S2). ${ }^{[7]}$ From these data it is evident that simple models, such as the Hammett equation, are not able to correctly predict the reactivity of all of these tetrazines. Using density functional theory (DFT) we investigated the reactivity of 22 tetrazines (2-9a, 2-9b, 2c, and 5-9c), selected out of our library, with trans-cyclooctene in more detail. Gas-phase $\omega \mathrm{B} 97 \mathrm{X}-\mathrm{D} / \mathrm{def} 2-\mathrm{TZVPD}$ calculated reaction barriers show no correlation to the logarithm of measured rate constants (Figure 4A). For compounds with substituents in 6-position the calculated barriers are underestimated by several $\mathrm{kcal} / \mathrm{mol}$ compared to 5- and 6-substituted derivatives. The reactivity of $\mathbf{8 a}$ is also overestimated due to the use of the protonated species in these gas-phase calculations. Including water solvent effects through the implicit SMD model and using deprotonated 8a, however, leads to a good correlation and a predictive model for the reactivity of such tetrazines (Figure 4B). 
A)

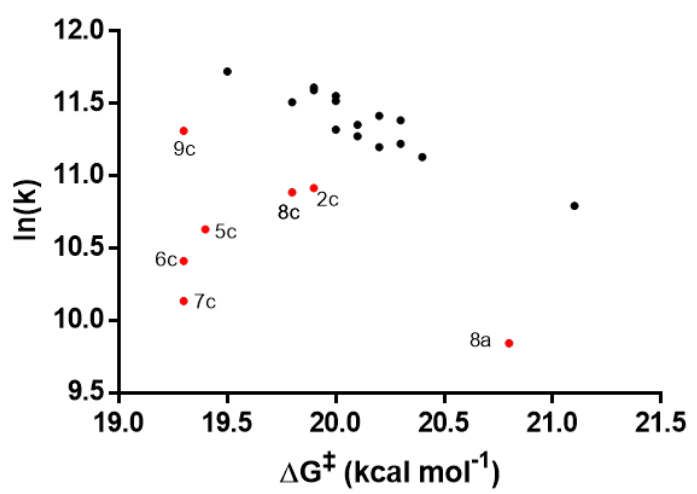

B)

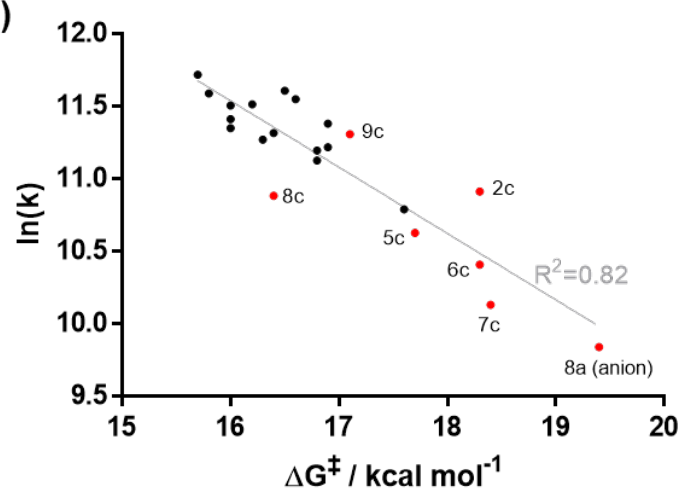

Figure 4. A) Correlation between gas-phase calculated $\Delta \mathrm{G}^{\ddagger}$ values and the natural logarithm of the experimental rate constant. B) Correlation between SMD (water) calculated $\Delta \mathrm{G}^{*}$ values and the natural logarithm of the experimental rate constant.

The discrepancy between calculations in the gas phase and in solution reveals two interesting effects. First, the intrinsic (gas-phase) reactivity of 6-substituted derivatives is unexpectedly high compared to the respective 4- and 5-substituted isomers. One would expect that a bulky substituent in orthoposition to the tetrazine leads to lower IEDDA reactivity due to increased steric demand, but the opposite seems to be the case. This high intrinsic reactivity is likely based on a distortion-lowering effect. Due to the substituent the two aromatic systems are not coplanar in the reactant, which leads to a reduced energy penalty when forcing the tetrazine into transition state geometry. This effect was recently described for 2-pyridyl-substituted tetrazines, showing that intramolecular repulsion of the lone-pairs of two nitrogen atoms leads to a similar effect. ${ }^{[21]}$ Second, a strong solvent effect is observed in which inclusion of a solvent model lowers the barriers of 6-substituted tetrazines less than those of 4- and 5-substituted derivatives. In the rate-limiting step (Tz/TCO Diels-Alder cycloaddition) a polarized transition state is formed, which can be stabilized through the solvent. More polar solvents and hydrogen bonds stabilize the transition state better, which leads to a lowered barrier and the observed acceleration in water compared to aprotic, less polar solvents. In case of 6substituted tetrazines the substituent partially blocks solvent access to the polarized part of the transition state structure, thus leading to less stabilization. While the average stabilization through introduction of the SMD water model for 4- and 5- substituted derivatives is $3.7 \mathrm{kcal} / \mathrm{mol}$ (with a range of 3.4 to $4.2 \mathrm{kcal} / \mathrm{mol}$ ), the average stabilization for 6 -substituted tetrazines is only $1.8 \mathrm{kcal} / \mathrm{mol}$ (ranging from 0.9 to $3.4 \mathrm{kcal} / \mathrm{mol}$ ). A larger size of the substituent also results in less solvent access, which leads to the observed correlation between second order rate constants and steric parameters. A more detailed investigation of these effects is ongoing and will be reported in due course. 
For 4-substituted phenyltetrazines the reactivity is purely FMO controlled, as already suspected due to the good correlation with Hammett constants. Since the reaction is an inverse electron-demand Diels-Alder cycloaddition, the relevant orbital of the tetrazine is the LUMO+1. ${ }^{[11 \mathrm{a}]}$ Plotting LUMO+1 energies against calculated barriers reveals an excellent correlation (Figure 5A). Calculation of orbital energies are therefore sufficient to estimate the reactivity of these tetrazines. For 5-and 6-substituted compounds other effects come into play and FMO interactions are not indicative for the reactivity (Figure 5B and 5C). Therefore, an analysis of the reaction barrier must be conducted to correctly predict reactivity.
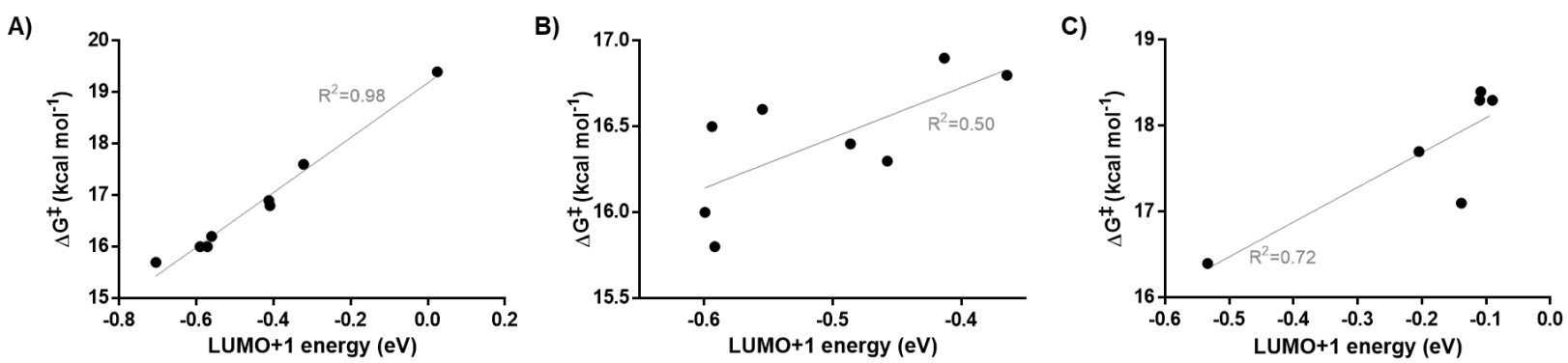

Figure 5. Correlation between LUMO+1 energies and calculated $\Delta \mathrm{G}^{\star}$ for A) 4-substituted, B) 5-substituted, and C) 6substituted derivatives.

\section{Conclusion}

Overall, our study sheds new light on the key parameters that affect the bioorthogonal reactivity of substituted fluorinated aryltetrazines. Kinetic investigations using a library of synthesized compounds revealed a substantial difference between the relative reactivities observed in $\mathrm{CH}_{3} \mathrm{CN}$ and in DPBS. This discrepancy is higher for ionic compounds and shows the importance of performing kinetic measurements in aqueous solution. Furthermore, the substitution pattern on the phenyl moiety of the aryltetrazine scaffold plays a crucial role. In the case of 4-substituted phenyltetrazines, the reactivity is mainly FMO controlled and can be easily predicted based on Hammett $\delta_{\mathrm{M}}$ parameters. In the case of 5- and 6-substituted phenyltetrazines more detailed studies are required. In particular, the reactivities of 5-substitued analogues can be well explained by DFT studies in the gas phase, while for the 6-substitued isomers the solvent needs to be taken into consideration. Our results indicate that the substituents at 6-position prevent the stabilization effect of the solvent on the polarized transition state resulting in a lower reactivity. Considering the recent development of radiolabeled ${ }^{18} \mathrm{~F}$ phenyltetrazines with improved bioorthogonal performance, our findings will aid the design of nextgeneration tetrazines for in vivo pretargeting. ${ }^{[7 e, 10,16]}$ 


\section{Experimental Section}

\section{Chemistry}

All reagents and solvents were dried prior to use according to standard methods. Commercial reagents were used without further purification. Analytical TLC was performed using silica gel 60 F254 (Merck) with detection by UV absorption and/or by charring following immersion in a 7\% ethanolic solution of sulfuric acid or $\mathrm{KMnO}_{4}$-solution $\left(1.5 \mathrm{~g}\right.$ of $\mathrm{KMnO}_{4}, 10 \mathrm{~g} \mathrm{~K}_{2} \mathrm{CO}_{3}$, and $1.25 \mathrm{~mL} 10 \% \mathrm{NaOH}$ in $200 \mathrm{~mL}$ water). Purification of compounds was carried out by column chromatography on silica gel (40-60 $\mu \mathrm{m}, 60 \AA$ ) or employing a CombiFlash NextGen $300+$ (Teledyne ISCO). ${ }^{1} \mathrm{H}$ and ${ }^{13} \mathrm{C}$ NMR spectra were recorded on Brucker (400 and $600 \mathrm{MHz}$ instruments), using Chloroform- $d$, Methanol$d_{4}$ or DMSO- $d_{6}$ as deuterated solvent and with the residual solvent as the internal reference. For all NMR experiences the deuterated solvent signal was used as the internal lock. Chemical shifts are reported in $\delta$ parts per million (ppm). Coupling constants $(J$ values) are given in Hertz $(\mathrm{Hz})$. Multiplicities of ${ }^{1} \mathrm{H}$ NMR signals are reported as follows: s, singlet; $\mathrm{d}$, doublet; dd, doublet of doublets; ddd, doublet of doublets of doublets; dt, doublet of triplets; t, triplet; q, quartet; m, multiplet; br, broad signal. NMR spectra of all compounds are reprocessed in MestReNova software (version 12.0.22023) from original FID’s files. Mass spectra analysis was performed using MS-Acquity-A: Waters Acquity UPLC with QDa-detector.

\section{Synthesis}

\section{3-(3-Fluoro-4-methylphenyl)-1,2,4,5-tetrazine (2a)}

The compound was obtained following the literature procedure. ${ }^{[15]} 3$-Fluoro-4-methylbenzonitrile (0.54 g, $4.00 \mathrm{mmol}), \mathrm{CH}_{2} \mathrm{Cl}_{2}(4.00 \mathrm{mmol}, 0.256 \mathrm{~mL})$, sulfur $(0.256 \mathrm{~g}, 1.00 \mathrm{mmol})$ and ethanol (4.0 $\mathrm{mL})$ were mixed together in a $20 \mathrm{ml}$ microwave reaction tube. Hydrazine monohydrate $(1.6 \mathrm{~mL}, 32.00$ mmol) was added slowly with stirring afterwards. The vessel was sealed, and the reaction mixture was heated to $50{ }^{\circ} \mathrm{C}$ for 24 hours. Then $3 \mathrm{ml}$ of $\mathrm{CH}_{2} \mathrm{Cl}_{2}$ and sodium nitrite $(2.76 \mathrm{~g}, 40.00 \mathrm{mmol})$ in $40 \mathrm{ml}$ of $\mathrm{H}_{2} \mathrm{O}$ were added to the mixture. Excess acetic acid $(14 \mathrm{~mL})$ was then added slowly during which the solution turned bright red in color. The reaction mixture was extracted with $\mathrm{CH}_{2} \mathrm{Cl}_{2}(3 \times 30$ $\mathrm{mL}$ ). The organic phase was dried over anhydrous $\mathrm{MgSO}_{4}$, filtered and concentrated under reduced pressure. The resulting residue was purified using flash chromatography $(95 / 5 \mathrm{Heptane} / \mathrm{EtOAc})$ to yield $0.21 \mathrm{~g}(28 \%)$ of $\mathbf{2 a}$ as a red solid. $\mathrm{Rf}=0.4$ (Heptane/EtAOc 80/20); ${ }^{1} \mathrm{H}$ NMR (400 MHz, Chloroform- $d$ ) $\delta 10.21$ (s, 1H), 8.33 (dd, $J=8.0,1.7 \mathrm{~Hz}, 1 \mathrm{H}), 8.27$ (dd, $J=10.5,1.7 \mathrm{~Hz}, 1 \mathrm{H}), 7.43$ $(\mathrm{t}, J=7.8 \mathrm{~Hz}, 1 \mathrm{H}), 2.41(\mathrm{~s}, 3 \mathrm{H}) ;{ }^{13} \mathrm{C}$ NMR $(101 \mathrm{MHz}$, Chloroform- $d) \delta 165.80,161.82(\mathrm{~d}, J=246.1$ $\mathrm{Hz}), 157.83,132.50$ (d, $J=8.3 \mathrm{~Hz}), 131.11$ (d, $J=8.3 \mathrm{~Hz}), 130.94$ (d, $J=17.4 \mathrm{~Hz}), 123.78$ (d, $J=$ $3.5 \mathrm{~Hz}), 114.69$ (d, $J=25.1 \mathrm{~Hz}), 14.92$ (d, $J=3.4 \mathrm{~Hz})$; MS (ESI) m/z [M + H] $]^{+}$191.11. 


\section{3-(3-Fluoro-5-methylphenyl)-1,2,4,5-tetrazine (2b)}

The compound was obtained from 3-fluoro-5-methylbenzonitrile (0.54 g, $4.00 \mathrm{mmol})$ following the procedure employed for 2a. Purification by flash chromatography (95/5 Heptane/EtOAc) afforded $0.26 \mathrm{~g}(34 \%)$ of $\mathbf{2 b}$ as a red oil. $\mathrm{Rf}=0.39$ (Heptane/EtOAc 80/20); ${ }^{1} \mathrm{H}$ NMR (400 MHz, Chloroformd) $\delta 10.16(\mathrm{~s}, 1 \mathrm{H}), 8.19(\mathrm{~d}, J=1.4 \mathrm{~Hz}, 1 \mathrm{H}), 8.05(\mathrm{~d}, J=9.4 \mathrm{~Hz}, 1 \mathrm{H}), 7.10(\mathrm{~d}, J=9.2 \mathrm{~Hz}, 1 \mathrm{H}), 2.43$ $(\mathrm{s}, 3 \mathrm{H}) ;{ }^{13} \mathrm{C}$ NMR $(101 \mathrm{MHz}$, Chloroform- $d) \delta 165.83(\mathrm{~d}, J=3.4 \mathrm{~Hz}), 163.30(\mathrm{~d}, J=246.8 \mathrm{~Hz})$, 157.95, 141.81 (d, $J=7.7 \mathrm{~Hz}), 133.31$ (d, $J=8.9 \mathrm{~Hz}), 124.67$ (d, $J=2.7 \mathrm{~Hz}), 120.79$ (d, $J=21.2$ Hz), 112.29 (d, $J=24.3 \mathrm{~Hz}), 21.45$ (d, $J=1.8 \mathrm{~Hz})$; MS (ESI) m/z [M + H] : 191.08.

\section{3-(3-Fluoro-5-methylphenyl)-1,2,4,5-tetrazine (2c)}

The compound was obtained from 3-fluoro-6-methylbenzonitrile $(0.54 \mathrm{~g}, 4.00 \mathrm{mmol})$ following the procedure employed for 2a. Purification by flash chromatography (95/5 Heptane/EtOAc) afforded $0.12 \mathrm{~g}(16 \%)$ of $2 \mathrm{c}$ as a red solid. $\mathrm{Rf}=0.37$ (Heptane/EtOAc 90/10); ${ }^{1} \mathrm{H}$ NMR (600 MHz, Chloroform-d) $\delta 10.23(\mathrm{~s}, 1 \mathrm{H}), 7.72(\mathrm{dd}, J=8.5,3.2 \mathrm{~Hz}, 1 \mathrm{H}), 7.32-7.27$ (m, 1H), 7.08 (dd, $J=9.1$, $4.2 \mathrm{~Hz}, 1 \mathrm{H}), 3.90$ (s, 3H); ${ }^{13} \mathrm{C} \mathrm{NMR}(151 \mathrm{MHz}$, Chloroform- $d$ ) $\delta 167.77$ (d, $J=2.2 \mathrm{~Hz}), 157.08(\mathrm{~d}, J$ $=240.5 \mathrm{~Hz}), 156.99,154.97(\mathrm{~d}, J=2.1 \mathrm{~Hz}), 122.91(\mathrm{~d}, J=7.8 \mathrm{~Hz}), 120.08$ (d, $J=22.9 \mathrm{~Hz}), 118.48$ (d, $J=25.3 \mathrm{~Hz}), 113.94$ (d, $J=7.9 \mathrm{~Hz}), 56.96$; MS (ESI) m/z [M + H] $]^{+}: 191.09$.

\section{3-(3-Fluoro-4-(trifluoromethyl)phenyl)-1,2,4,5-tetrazine (3a)}

The compound was obtained from 3-fluoro-4-trifluoromethylbenzonitrile (0.76 g, $4.00 \mathrm{mmol})$ following the procedure employed for 2a. Purification by flash chromatography $(95 / 5$ Heptane/EtOAc) afforded $0.21 \mathrm{~g}(21 \%)$ of $\mathbf{3 a}$ as a red solid. $\mathrm{Rf}=0.41(80 / 20 \mathrm{Heptane} / \mathrm{EtOAc}) ;{ }^{1} \mathrm{H}$ NMR (400 MHz, Chloroform- $d$ ) $\delta 10.36(\mathrm{~s}, 1 \mathrm{H}), 8.65-8.54(\mathrm{~m}, 1 \mathrm{H}), 8.55-8.45(\mathrm{~m}, 1 \mathrm{H}), 7.90$ (t, $J$ $=7.6 \mathrm{~Hz}, 1 \mathrm{H}) ;{ }^{13} \mathrm{C}$ NMR $(101 \mathrm{MHz}$, Chloroform- $d$ ) $\delta 164.80(\mathrm{~d}, J=2.9 \mathrm{~Hz}), 160.26(\mathrm{dd}, J=258.1$, $2.1 \mathrm{~Hz}), 158.32,137.35(\mathrm{~d}, J=8.3 \mathrm{~Hz}), 130.90-126.53(\mathrm{~m}), 128.36(\mathrm{dd}, J=4.4,1.8 \mathrm{~Hz}), 123.75(\mathrm{~d}$, $J=4.1 \mathrm{~Hz}), 123.46,123.12-121.35(\mathrm{~m}), 120.76,116.57$ (d, $J=23.5 \mathrm{~Hz})$; MS (ESI) m/z [M + H] $]^{+}$ 245.08 .

\section{3-(3-Fluoro-5-(trifluoromethyl)phenyl)-1,2,4,5-tetrazine (3b)}

The compound was obtained from 3-fluoro-5-trifluoromethylbenzonitrile (0.76 g, $4.00 \mathrm{mmol})$ following the procedure employed for 2a. Purification by flash chromatography (95/5 Heptane/EtOAc) afforded $0.23 \mathrm{~g}(24 \%)$ of $\mathbf{3 b}$ as a red solid. $\mathrm{Rf}=0.39\left(\right.$ Heptane/EtOAc 80/20); ${ }^{1} \mathrm{H}$ NMR (400 MHz, Chloroform- $d$ ) $\delta 10.25(\mathrm{~s}, 1 \mathrm{H}), 8.65(\mathrm{~s}, 1 \mathrm{H}), 8.44(\mathrm{~d}, J=9.0 \mathrm{~Hz}, 1 \mathrm{H}), 7.53(\mathrm{~d}, J=$ $8.1 \mathrm{~Hz}, 1 \mathrm{H}) ;{ }^{13} \mathrm{C}$ NMR $(101 \mathrm{MHz}$, Chloroform- $d) \delta 164.72(\mathrm{~d}, J=3.0 \mathrm{~Hz}), 163.01(\mathrm{~d}, J=250.8 \mathrm{~Hz})$, 
$158.33,134.88(\mathrm{~d}, J=8.3 \mathrm{~Hz}), 133.95(\mathrm{qd}, J=34.1,7.9 \mathrm{~Hz}), 122.79(\mathrm{dd}, J=272.9,3.0 \mathrm{~Hz}), 121.41$ - $120.21(\mathrm{~m}), 118.41$ (d, $J=24.2 \mathrm{~Hz}), 117.20$ (dq, $J=24.6,3.7 \mathrm{~Hz})$; MS (ESI) m/z [M + H] : 245.07.

\section{(2-Fluoro-4-(1,2,4,5-tetrazin-3-yl)phenyl)methanamine (4a)}

\section{4-((1,3-Dioxoisoindolin-2-yl)methyl)-3-fluorobenzonitrile (18a)}

The compound was obtained following the literature procedure with minor modifications. ${ }^{[12]} 2$ (Bromomethyl)-3-fluorobenzonitrile (3.0 g, $14.01 \mathrm{mmol})$ was dissolved in DMF (20 $\mathrm{mL})$. Phthalimide potassium salt $(2.89 \mathrm{~g}, 15.41)$ was added and the mixture was stirred for $9 \mathrm{~h}$ at $130{ }^{\circ} \mathrm{C}$. After cooling to rt, the mixture was poured on ice. The solid obtained was filtered off. Ethyl acetate $(100 \mathrm{~mL})$ and water $(100 \mathrm{~mL})$ were added and the organic layer was separated. The organic phase was washed with water $(2 \times 50 \mathrm{~mL})$, dried over $\mathrm{MgSO}_{4}$, filtered and evaporated to give a light brown solid. Crystallization from EtOAc afforded $3.30 \mathrm{~g}(84 \%)$ of the desired compound as a white solid. $\mathrm{Rf}=0.24\left(\right.$ Heptane/EtOAc 70/30); ${ }^{1} \mathrm{H}$ NMR (400 MHz, Chloroform- $d$ ) $\delta 7.92-7.86(\mathrm{~m}, 2 \mathrm{H}), 7.81-$ 7.74 (m, 2H), $7.52-7.33$ (m, 3H), 4.98 (s, 2H); ${ }^{13} \mathrm{C}$ NMR (101 MHz, Chloroform-d) $\delta 167.55,159.99$ $(\mathrm{d}, J=252.2 \mathrm{~Hz}), 134.38,131.80,130.99(\mathrm{~d}, J=4.3 \mathrm{~Hz}), 129.06(\mathrm{~d}, J=14.9 \mathrm{~Hz}), 128.29(\mathrm{~d}, J=4.0$ Hz), 123.64, 119.34 (d, $J=24.9 \mathrm{~Hz}), 117.27$ (d, $J=2.9 \mathrm{~Hz}), 113.24$ (d, $J=9.5 \mathrm{~Hz}), 35.09$ (d, $J=4.6$ $\mathrm{Hz})$.

\section{(4-Cyano-2-fluorophenyl)methanaminium chloride (19a)}

The compound was obtained following the literature procedure with minor modifications. ${ }^{[12]}$ To a solution of 4-((1,3-dioxoisoindolin-2-yl)methyl)-3-fluorobenzonitrile (3.0 g, $10.70 \mathrm{mmol})$ in EtOH $(5 \mathrm{~mL})$ was added hydrazine hydrate $(5 \mathrm{~mL})$. The reaction was then refluxed for $2 \mathrm{~h}$ and a white precipitate was formed. The reaction was diluted with $\mathrm{NaOH}$ solution $(10 \%, 40 \mathrm{~mL})$ and extracted with EtOAc $(3 \times 30 \mathrm{~mL})$. The organic portion was dried over anhydrous $\mathrm{Na}_{2} \mathrm{SO}_{4}$, filtered and the solvent was evaporated to dryness under reduced pressure. The crude was solubilized in $\mathrm{Et}_{2} \mathrm{O}$, filtered and treated with $\mathrm{HCl}$ in $\mathrm{Et}_{2} \mathrm{O}(2 \mathrm{~mL}, 2 \mathrm{M})$. The solid obtained was filtered and recrystallized from $\mathrm{MeOH}$ to give $1.51 \mathrm{~g}(76 \%)$ of the desired compound as a yellow solid. ${ }^{1} \mathrm{H}$ NMR (400 MHz, DMSO$\left.d_{6}\right) \delta 8.72(\mathrm{~s}, 3 \mathrm{H}), 7.95(\mathrm{~d}, J=9.9 \mathrm{~Hz}, 1 \mathrm{H}), 7.90-7.77(\mathrm{~m}, 2 \mathrm{H}), 4.13(\mathrm{~s}, 2 \mathrm{H}) ;{ }^{13} \mathrm{C} \mathrm{NMR}(101 \mathrm{MHz}$, DMSO-d $)) 160.13(\mathrm{~d}, J=249.5 \mathrm{~Hz}), 132.78(\mathrm{~d}, J=3.9 \mathrm{~Hz}), 129.27(\mathrm{~d}, J=3.9 \mathrm{~Hz}), 127.73(\mathrm{~d}, J=$ $14.8 \mathrm{~Hz}), 119.87(\mathrm{~d}, J=25.6 \mathrm{~Hz}), 117.84(\mathrm{~d}, J=3.0 \mathrm{~Hz}), 113.38(\mathrm{~d}, J=10.3 \mathrm{~Hz}), 35.87$ (d, $J=4.4$ $\mathrm{Hz})$.

\section{Tert-butyl 4-cyano-2-fluorobenzylcarbamate (20a)}

(4-Cyano-2-fluorophenyl)methanaminium chloride (1.5 g, $8.04 \mathrm{mmol})$ and triethylamine $(2.35 \mathrm{~mL}$, $16.87 \mathrm{mmol})$ were dissolved in anhydrous $\mathrm{CH}_{2} \mathrm{Cl}_{2}(40 \mathrm{~mL})$ at $0^{\circ} \mathrm{C}$. To this stirred solution was added di-tert-butyl dicarbonate $(2.10 \mathrm{~g}, 9.64 \mathrm{mmol})$, and the reaction allowed to warm to room temperature 
and stirred for 12 hours. The reaction mixture was evaporated under reduced pressure, and the residue was re-dissolved in diethyl ether $(50 \mathrm{~mL})$, which was washed successively with $0.5 \mathrm{M}$ aq. $\mathrm{HCl}(2 \mathrm{x}$ $25 \mathrm{~mL})$, saturated $\mathrm{NaHCO}_{3}(2 \times 25 \mathrm{~mL})$ and brine $(25 \mathrm{~mL})$. The organic layer was dried over anhydrous $\mathrm{MgSO}_{4}$, filtered and evaporated under reduced pressure to give a white solid. The residue was purified by flash column chromatography (Heptane/EtOAc $=85 / 15)$ to afford $1.51 \mathrm{~g}(75 \%)$ of the desired compound as an orange solid. $\mathrm{Rf}=0.24$ (Heptane/EtOAc 80/20); ${ }^{1} \mathrm{H}$ NMR (400 MHz, Chloroform-d) $\delta 7.48-7.30(\mathrm{~m}, 2 \mathrm{H}), 7.22(\mathrm{~d}, J=9.4 \mathrm{~Hz}, 1 \mathrm{H}), 5.52(\mathrm{t}, J=6.4 \mathrm{~Hz}, 1 \mathrm{H}), 4.29(\mathrm{~d}, J=$ $6.4 \mathrm{~Hz}, 2 \mathrm{H}), 1.35$ (s, 9H); ${ }^{13} \mathrm{C}$ NMR (101 MHz, Chloroform- $d$ ) $\delta 159.86$ (d, $\left.J=249.8 \mathrm{~Hz}\right), 155.96$, $132.56(\mathrm{~d}, J=14.5 \mathrm{~Hz}), 130.10(\mathrm{~d}, J=5.0 \mathrm{~Hz}), 128.18(\mathrm{~d}, J=3.9 \mathrm{~Hz}), 118.69(\mathrm{~d}, J=25.0 \mathrm{~Hz}), 117.44$ (d, $J=2.9 \mathrm{~Hz}), 112.10(\mathrm{~d}, J=9.5 \mathrm{~Hz}), 38.04,28.21$.

Tert-butyl 2-fluoro-4-(1,2,4,5-tetrazin-3-yl)benzylcarbamate (28a)

The compound was obtained from tert-butyl 4-cyano-2-fluorobenzylcarbamate (0.76 g, $4.00 \mathrm{mmol})$ following the procedure employed for 2a. Purification by flash chromatography (95/5 Heptane/EtOAc) afforded $0.39 \mathrm{~g}(21 \%)$ of the desire compound as a red solid. $\mathrm{Rf}=0.25$ (Heptane/EtOAc 80/20); ${ }^{1} \mathrm{H}$ NMR (400 MHz, Chloroform- $d$ ) $\delta 10.25$ (s, 1H), 8.41 (dd, $J=8.1,1.6$ $\mathrm{Hz}, 1 \mathrm{H}), 7.60(\mathrm{t}, J=7.7 \mathrm{~Hz}, 1 \mathrm{H}), 5.11(\mathrm{~s}, 1 \mathrm{H}), 4.49$ (d, $J=6.3 \mathrm{~Hz}, 2 \mathrm{H}), 1.48(\mathrm{~s}, 9 \mathrm{H}) ;{ }^{13} \mathrm{C} \mathrm{NMR}(101$ MHz, Chloroform- $d$ ) $\delta 165.53(\mathrm{~d}, J=3.0 \mathrm{~Hz}), 161.21(\mathrm{~d}, J=247.6 \mathrm{~Hz}), 157.94,155.82,132.59$ (d, $J$ $=8.5 \mathrm{~Hz}), 131.64(\mathrm{~d}, J=15.0 \mathrm{~Hz}), 130.48,124.13,114.98$ (d, $J=24.5 \mathrm{~Hz}), 79.97,38.57,28.36$.

(2-Fluoro-4-(1,2,4,5-tetrazin-3-yl)phenyl)methanamine

To a solution of tert-butyl 2-fluoro-4-(1,2,4,5-tetrazin-3-yl)benzylcarbamate $(0.300 \mathrm{~g}, 0.98 \mathrm{mmol})$ in $\mathrm{CH}_{2} \mathrm{Cl}_{2}(20 \mathrm{~mL})$ was added a solution of $\mathrm{HCl}$ in dioxane $(4.0 \mathrm{M}, 10.0 \mathrm{~mL})$. The mixture was stirred at room temperature for $2 \mathrm{~h}$. The reaction was then concentrated under reduced pressure to give 0.23 $\mathrm{g}(97 \%)$ of $\mathrm{HCl}$ salt of $\mathbf{4 a}$ as a pink solid. ${ }^{1} \mathrm{H}$ NMR $(400 \mathrm{MHz}$, Methanol-d $) \delta 10.34(\mathrm{~s}, 1 \mathrm{H}), 8.43$ $(\mathrm{dd}, J=8.0,1.6 \mathrm{~Hz}, 1 \mathrm{H}), 8.33(\mathrm{dd}, J=10.9,1.7 \mathrm{~Hz}, 1 \mathrm{H}), 7.74(\mathrm{t}, J=7.8 \mathrm{~Hz}, 1 \mathrm{H}), 4.27(\mathrm{~s}, 2 \mathrm{H}) ;{ }^{13} \mathrm{C}$ NMR $\left(101 \mathrm{MHz}\right.$, Methanol- $\left.d_{4}\right) \delta 165.15,161.44(\mathrm{~d}, J=248.4 \mathrm{~Hz}), 158.27,135.65(\mathrm{~d}, J=8.5 \mathrm{~Hz})$, $131.96(\mathrm{~d}, J=3.5 \mathrm{~Hz}), 124.80(\mathrm{~d}, J=15.3 \mathrm{~Hz}), 124.08$ (d, $J=3.7 \mathrm{~Hz}), 114.72$ (d, $J=24.5 \mathrm{~Hz}), 36.45$ $(\mathrm{d}, J=4.2 \mathrm{~Hz}) ; \mathrm{MS}(\mathrm{ESI}) \mathrm{m} / \mathrm{z}[\mathrm{M}+\mathrm{H}]^{+}: 206.10$.

\section{(3-Fluoro-5-(1,2,4,5-tetrazin-3-yl)phenyl)methanamine (4b)}

\section{5-((1,3-Dioxoisoindolin-2-yl)methyl)-3-fluorobenzonitrile (18b)}

The compound was obtained from 3-(bromomethyl)-5-fluorobenzonitrile (1.25 g, $5.84 \mathrm{mmol})$ following the procedure reported above for 18a. Crystallization from EtOAc afforded $1.62 \mathrm{~g} \mathrm{(99 \% )}$ of the desired compound as a white solid. Rf $=0.26$ (Heptane/EtOAc 70/30); ${ }^{1} \mathrm{H}$ NMR (400 MHz, Chloroform- $d$ ) $\delta 7.93-7.87(\mathrm{~m}, 2 \mathrm{H}), 7.81-7.74(\mathrm{~m}, 2 \mathrm{H}), 7.53(\mathrm{~d}, J=1.5 \mathrm{~Hz}, 1 \mathrm{H}), 7.41(\mathrm{dt}, J=9.0$, 
$2.0 \mathrm{~Hz}, 1 \mathrm{H}), 7.32-7.25$ (m, 1H), 4.87 (s, 2H); ${ }^{13} \mathrm{C}$ NMR (101 MHz, Chloroform-d) $\delta$ 167.66, 162.27 (d, $J=251.4 \mathrm{~Hz}), 140.44(\mathrm{~d}, J=7.6 \mathrm{~Hz}), 134.43,131.76,128.05$ (d, $J=3.5 \mathrm{~Hz}), 123.70,120.67$ (d, $J=21.8 \mathrm{~Hz}), 118.64(\mathrm{~d}, J=24.6 \mathrm{~Hz}), 117.19(\mathrm{~d}, J=3.3 \mathrm{~Hz}), 114.23(\mathrm{~d}, J=9.7 \mathrm{~Hz}), 40.41(\mathrm{~d}, J=1.9$ $\mathrm{Hz})$.

\section{(3-Cyano-5-fluorophenyl)methanaminium chloride (19b)}

The compound was obtained from 5-((1,3-dioxoisoindolin-2-yl)methyl)-3-fluorobenzonitrile (1.6 g, $5.71 \mathrm{mmol}$ ) following the procedure reported above 19a. Recrystallization from $\mathrm{MeOH} / \mathrm{Et}_{2} \mathrm{O}$ afforded $0.71 \mathrm{~g}(67 \%)$ of the desired compound as a yellow solid. ${ }^{1} \mathrm{H}$ NMR (400 MHz, Methanol- $d_{4}$ ) $\delta 7.76(\mathrm{t}, J=1.4 \mathrm{~Hz}, 1 \mathrm{H}), 7.73-7.62(\mathrm{~m}, 2 \mathrm{H}), 4.26(\mathrm{~s}, 2 \mathrm{H}) ;{ }^{13} \mathrm{C}$ NMR (101 MHz, Methanol- $\left.d_{4}\right) \delta$ $163.76(\mathrm{~d}, J=249.8 \mathrm{~Hz}), 138.91(\mathrm{~d}, J=8.0 \mathrm{~Hz}), 130.12(\mathrm{~d}, J=3.8 \mathrm{~Hz}), 122.40(\mathrm{~d}, J=22.7 \mathrm{~Hz})$, $120.79(\mathrm{~d}, J=25.2 \mathrm{~Hz}), 118.01,115.74(\mathrm{~d}, J=10.1 \mathrm{~Hz}), 43.01(\mathrm{~d}, J=1.5 \mathrm{~Hz})$.

Tert-butyl 3-cyano-5-fluorobenzylcarbamate (20b)

The compound was obtained from (3-cyano-5-fluorophenyl)methanaminium chloride (0.71 g, 3.75 mmol) following the procedure reported above for 20a. Purification by flash chromatography $($ Heptane/EtOAc $=85 / 15)$ afforded $0.51 \mathrm{~g}(54 \%)$ of the desired compound as an orange solid. $\mathrm{Rf}=$ 0.26 (Heptane/EtOAc 80/20); ${ }^{1} \mathrm{H}$ NMR (400 MHz, Chloroform-d) $\delta 7.32$ (s, 1H), 7.26 - 7.10 (m, 2H), $5.57(\mathrm{t}, J=6.2 \mathrm{~Hz}, 1 \mathrm{H}), 4.26(\mathrm{~d}, J=6.2 \mathrm{~Hz}, 2 \mathrm{H}), 1.39$ (s, 9H); ${ }^{13} \mathrm{C}$ NMR (101 MHz, Chloroform- $d$ ) $\delta 162.26(\mathrm{~d}, J=250.4 \mathrm{~Hz}), 156.01,144.21(\mathrm{~d}, J=7.5 \mathrm{~Hz}), 126.53(\mathrm{~d}, J=3.2 \mathrm{~Hz}), 119.09(\mathrm{~d}, J=21.7$ $\mathrm{Hz}), 117.56$ (d, $J=27.6 \mathrm{~Hz}), 117.45,113.65$ (d, $J=9.7 \mathrm{~Hz}), 79.98,43.34,28.23$.

\section{Tert-butyl 3-fluoro-5-(1,2,4,5-tetrazin-3-yl)benzylcarbamate (28b)}

The compound was obtained from tert-butyl 3-cyano-5-fluorobenzylcarbamate $(0.40 \mathrm{~g}, 1.60 \mathrm{mmol})$ following the procedure employed for 2a. Purification by flash chromatography (95/5 Heptane/EtOAc) afforded $0.16 \mathrm{~g}(33 \%)$ of the desired compound as a red solid. $\mathrm{Rf}=0.26$ (Heptane/EtOAc 80/20); ${ }^{1} \mathrm{H}$ NMR (400 MHz, Chloroform-d) $\delta 10.26$ (s, 1H), 8.35 (s, 1H), 8.20 (dt, $J=9.2,2.0 \mathrm{~Hz}, 1 \mathrm{H}), 7.35-7.28(\mathrm{~m}, 1 \mathrm{H}), 5.21(\mathrm{t}, J=5.7 \mathrm{~Hz}, 1 \mathrm{H}), 4.45(\mathrm{~d}, J=5.7 \mathrm{~Hz}, 2 \mathrm{H}), 1.46(\mathrm{~s}$, 9H); ${ }^{13} \mathrm{C}$ NMR (101 MHz, Chloroform- $d$ ) $\delta 165.56$ (d, $\left.J=3.3 \mathrm{~Hz}\right), 163.46$ (d, $\left.J=248.1 \mathrm{~Hz}\right), 158.00$, $143.45(\mathrm{~d}, J=5.8 \mathrm{~Hz}), 133.75(\mathrm{~d}, J=8.6 \mathrm{~Hz}), 122.49,118.86(\mathrm{~d}, J=22.2 \mathrm{~Hz}), 113.91(\mathrm{~d}, J=24.3$ $\mathrm{Hz}), 81.41,80.04,43.99,28.35$.

\section{(3-Fluoro-5-(1,2,4,5-tetrazin-3-yl)phenyl)methanamine}

To a solution of tert-butyl 3-fluoro-5-(1,2,4,5-tetrazin-3-yl)benzylcarbamate $(0.140 \mathrm{~g}, 0.46 \mathrm{mmol})$ in $\mathrm{CH}_{2} \mathrm{Cl}_{2}(10 \mathrm{~mL})$ was added a solution of $\mathrm{HCl}$ in dioxane $(4.0 \mathrm{M}, 5.0 \mathrm{~mL})$. The mixture was stirred at room temperature for $2 \mathrm{~h}$. The reaction was then concentrated under reduced pressure to $0.07 \mathrm{~g}(63 \%)$ of $\mathrm{HCl}$ salt of $\mathbf{4 b}$ as a pink solid. ${ }^{1} \mathrm{H}$ NMR $\left(400 \mathrm{MHz}\right.$, Methanol- $\left.d_{4}\right) \delta 10.33(\mathrm{~s}, 1 \mathrm{H}), 8.48(\mathrm{~d}, J=1.6$ $\mathrm{Hz}, 1 \mathrm{H}), 8.26(\mathrm{dt}, J=9.4,1.8 \mathrm{~Hz}, 1 \mathrm{H}), 7.52(\mathrm{dt}, J=9.0,1.9 \mathrm{~Hz}, 1 \mathrm{H}), 4.23(\mathrm{~s}, 2 \mathrm{H}) ;{ }^{13} \mathrm{C} \mathrm{NMR}(101$ 
MHz, Methanol- $\left.d_{4}\right) \delta 165.15(\mathrm{~d}, J=3.3 \mathrm{~Hz}), 163.32(\mathrm{~d}, J=247.4 \mathrm{~Hz}), 158.31,137.02(\mathrm{~d}, J=8.0$ Hz), 135.37 (d, $J=8.5 \mathrm{~Hz}), 124.09$ (d, $J=3.1 \mathrm{~Hz}), 119.87$ (d, $J=23.0 \mathrm{~Hz}), 114.92$ (d, $J=24.3 \mathrm{~Hz})$, 42.20; MS (ESI) m/z [M+ H] : 206.11.

\section{(4-Fluoro-2-(1,2,4,5-tetrazin-3-yl)phenyl)methanamine (4c)}

\section{2-((1,3-Dioxoisoindolin-2-yl)methyl)-5-fluorobenzonitrile (18c)}

The compound was obtained from 2-(bromomethyl)-5-fluorobenzonitrile (2.0 g, $9.34 \mathrm{mmol})$ following the procedure reported above for 18a. Crystallization from EtOAc afforded $2.60 \mathrm{~g} \mathrm{(99 \% )}$ of the desired compound as a white solid. $\mathrm{Rf}=0.28$ (Heptane/EtOAc 70/30); ${ }^{1} \mathrm{H}$ NMR (400 MHz, DMSO-d $d_{6} \delta 8.01-7.76(\mathrm{~m}, 5 \mathrm{H}), 7.60-7.42(\mathrm{~m}, 2 \mathrm{H}), 4.93(\mathrm{~s}, 2 \mathrm{H}) ;{ }^{13} \mathrm{C}$ NMR (101 MHz, DMSO$\left.d_{6}\right) \delta 172.71,165.87(\mathrm{~d}, J=246.6 \mathrm{~Hz}), 141.61(\mathrm{~d}, J=3.4 \mathrm{~Hz}), 139.82,136.79,136.22(\mathrm{~d}, J=8.8 \mathrm{~Hz})$, 128.53, 126.28 (d, $J=21.5 \mathrm{~Hz}), 125.05$ (d, $J=26.3 \mathrm{~Hz}), 124.94,121.23$ (d, $J=2.8 \mathrm{~Hz}), 117.23$ (d, $J$ $=10.0 \mathrm{~Hz}), 44.14$.

\section{(2-Cyano-4-fluorophenyl)methanaminium chloride (19c)}

The compound was obtained from 2-((1,3-dioxoisoindolin-2-yl)methyl)-5-fluorobenzonitrile (2.6 g, $9.23 \mathrm{mmol}$ ) following the procedure reported above 19a. Recrystallization from $\mathrm{MeOH} / \mathrm{Et}_{2} \mathrm{O}$ afforded $1.41 \mathrm{~g} \mathrm{(81 \% )}$ of the desired compound as a yellow solid. ${ }^{1} \mathrm{H}$ NMR (400 MHz, DMSO- $\left.d_{6}\right) \delta$ $10.53(\mathrm{~s}, 1 \mathrm{H}), 9.79(\mathrm{~s}, 1 \mathrm{H}), 9.37(\mathrm{~s}, 1 \mathrm{H}), 8.18(\mathrm{dd}, J=8.6,2.5 \mathrm{~Hz}, 1 \mathrm{H}), 7.82(\mathrm{dd}, J=8.5,4.8 \mathrm{~Hz}, 1 \mathrm{H})$, $7.68(\mathrm{td}, J=8.9,2.5 \mathrm{~Hz}, 1 \mathrm{H}), 4.80(\mathrm{~s}, 2 \mathrm{H}) ;{ }^{13} \mathrm{C}$ NMR $\left(101 \mathrm{MHz}, \mathrm{DMSO}-d_{6}\right) \delta 163.10(\mathrm{~d}, J=3.8 \mathrm{~Hz})$, $162.20(\mathrm{~d}, J=244.3 \mathrm{~Hz}), 140.95,130.40$ (d, $J=10.2 \mathrm{~Hz}), 126.44(\mathrm{~d}, J=9.0 \mathrm{~Hz}), 121.63$ (d, $J=23.5$ $\mathrm{Hz}), 110.86,51.40$.

\section{Tert-butyl 2-cyano-4-fluorobenzylcarbamate (20c)}

The compound was obtained from (2-cyano-4-fluorophenyl)methanaminium chloride (1.0 g, 5.36 mmo) following the procedure reported above for 20a. Purification by flash chromatography $($ Heptane/EtOAc $=85 / 15)$ afforded $0.85 \mathrm{~g}(63 \%)$ of the desired compound as an orange solid. $\mathrm{Rf}=$ 0.29 (Heptane/EtOAc 80/20); ${ }^{1} \mathrm{H}$ NMR (400 MHz, Chloroform- $d$ ) $\delta 7.65$ (d, $\left.J=8.1 \mathrm{~Hz}, 1 \mathrm{H}\right), 7.33$ $(\mathrm{dd}, J=8.4,4.6 \mathrm{~Hz}, 1 \mathrm{H}), 7.22(\mathrm{td}, J=8.6,2.5 \mathrm{~Hz}, 1 \mathrm{H}), 4.71(\mathrm{~s}, 2 \mathrm{H}), 1.58(\mathrm{~s}, 9 \mathrm{H}) ;{ }^{13} \mathrm{C} \mathrm{NMR}(101$ MHz, Chloroform- $d$ ) $\delta 162.89$ (d, $J=246.9 \mathrm{~Hz}), 158.04,152.55,135.85,133.99,124.05$ (d, $J=8.5$ $\mathrm{Hz}), 119.59$ (d, $J=23.8 \mathrm{~Hz}), 110.32$ (d, $J=23.7 \mathrm{~Hz}), 83.19,50.83,28.24$.

Tert-butyl 4-fluoro-2-(1,2,4,5-tetrazin-3-yl)benzylcarbamate (28c)

The compound was obtained from tert-butyl 2-cyano-4-fluorobenzylcarbamate (0.55 g, $2.20 \mathrm{mmol})$ following the procedure employed for 2a. Purification by flash chromatography $(95 / 5$ Heptane/EtOAc) afforded $0.100 \mathrm{~g}(15 \%)$ of the desire compound as a red solid. $\mathrm{Rf}=0.29$ (Heptane/EtOAc 80/20); ${ }^{1} \mathrm{H}$ NMR (400 MHz, Chloroform- $d$ ) $\delta 10.30$ (s, 1H), $8.15-7.89$ (m, 1H), 
$7.70(\mathrm{~d}, J=7.0 \mathrm{~Hz}, 1 \mathrm{H}), 7.31(\mathrm{td}, J=8.1,2.9 \mathrm{~Hz}, 1 \mathrm{H}), 5.56(\mathrm{~s}, 1 \mathrm{H}), 4.49(\mathrm{~d}, J=6.5 \mathrm{~Hz}, 2 \mathrm{H}), 1.41$ (s, $9 \mathrm{H}) ;{ }^{13} \mathrm{C}$ NMR (101 MHz, Chloroform- $d$ ) $\delta 167.76,162.12$ (d, $\left.J=247.9 \mathrm{~Hz}\right), 157.11,155.72,135.75$, 133.81, 132.47, 119.54 (d, $J=21.0 \mathrm{~Hz}), 117.91$ (d, $J=23.8 \mathrm{~Hz}), 79.59,42.61,28.39$.

(4-Fluoro-2-(1,2,4,5-tetrazin-3-yl)phenyl)methanamine

To a solution of tert-butyl 4-fluoro-2-(1,2,4,5-tetrazin-3-yl)benzylcarbamate $(0.10 \mathrm{~g}, 0.33 \mathrm{mmol})$ in $\mathrm{CH}_{2} \mathrm{Cl}_{2}(10 \mathrm{~mL})$ was added a solution of $\mathrm{HCl}$ in dioxane $(4.0 \mathrm{M}, 5.0 \mathrm{~mL})$. The mixture was stirred at room temperature for $2 \mathrm{~h}$. The reaction was then concentrated under reduced pressure to $0.045 \mathrm{~g}$ $(57 \%)$ of $\mathrm{HCl}$ salt of $4 \mathbf{c}$ as a pink solid. ${ }^{1} \mathrm{H}$ NMR $\left(400 \mathrm{MHz}\right.$, Methanol- $\left.d_{4}\right) \delta 10.42(\mathrm{~s}, 1 \mathrm{H}), 8.15(\mathrm{dd}$, $J=9.7,2.8 \mathrm{~Hz}, 1 \mathrm{H}), 7.74(\mathrm{dd}, J=8.6,5.4 \mathrm{~Hz}, 1 \mathrm{H}), 7.44(\mathrm{td}, J=8.2,2.9 \mathrm{~Hz}, 1 \mathrm{H}), 4.35(\mathrm{~s}, 2 \mathrm{H}) ;{ }^{13} \mathrm{C}$ NMR (101 MHz, Methanol- $\left.d_{4}\right) \delta 166.62(\mathrm{~d}, J=2.7 \mathrm{~Hz}), 163.29(\mathrm{~d}, J=249.0 \mathrm{~Hz}), 157.59,135.47$ (d, $J=8.5 \mathrm{~Hz}), 134.74(\mathrm{~d}, J=8.4 \mathrm{~Hz}), 129.06(\mathrm{~d}, J=3.8 \mathrm{~Hz}), 119.25(\mathrm{~d}, J=21.6 \mathrm{~Hz}), 117.86(\mathrm{~d}, J=$ 24.9 Hz), 41.22; MS (ESI) m/z [M + H] $]^{+}$206.07.

\section{3-(4-Chloro-3-fluorophenyl)-1,2,4,5-tetrazine (5a)}

The compound was obtained from 3-fluoro-4-chlorobenzonitrile (0.62 g, $4.00 \mathrm{mmol})$ following the procedure employed for 2a. Purification by flash chromatography (95/5 Heptane/EtOAc) afforded $0.18 \mathrm{~g}(21 \%)$ of $\mathbf{5 a}$ as a red solid. $\mathrm{Rf}=0.41$ (Heptane/EtOAc 80/20); ${ }^{1} \mathrm{H}$ NMR $(400 \mathrm{MHz}$, Chloroformd) $\delta 10.27(\mathrm{~s}, 1 \mathrm{H}), 8.37(\mathrm{dd}, J=9.3,2.0 \mathrm{~Hz}, 1 \mathrm{H}), 8.31(\mathrm{ddd}, J=8.4,2.0,0.7 \mathrm{~Hz}, 1 \mathrm{H}), 7.80(\mathrm{dd}, J=$ 8.4, 6.9 Hz, 1H); ${ }^{13} \mathrm{C}$ NMR (101 MHz, Chloroform- $d$ ) $\delta 165.25(\mathrm{~d}, J=2.7 \mathrm{~Hz}), 159.76(\mathrm{~d}, J=249.0$ Hz), 158.03, 134.75, 132.79 (d, $J=7.5 \mathrm{~Hz}), 124.79$ (d, $J=3.7 \mathrm{~Hz}), 115.93$ (d, $J=25.1 \mathrm{~Hz}), 115.17$ $(\mathrm{d}, J=21.2 \mathrm{~Hz}) ; \mathrm{MS}(\mathrm{ESI}) \mathrm{m} / \mathrm{z}[\mathrm{M}+\mathrm{H}]^{+}: 211.02$.

\section{3-(5-Chloro-3-fluorophenyl)-1,2,4,5-tetrazine (5b)}

The compound was obtained from 3-fluoro-5-chlorobenzonitrile (0.62 g, $4.00 \mathrm{mmol})$ following the procedure employed for 2a. Purification by flash chromatography (95/5 Heptane/EtOAc) afforded $0.22 \mathrm{~g}(26 \%)$ of $\mathbf{5 b}$ as a red solid. $\mathrm{Rf}=0.38$ (Heptane/EtOAc 80/20); ${ }^{1} \mathrm{H}$ NMR (400 MHz, Chloroformd) $\delta 10.22(\mathrm{~s}, 1 \mathrm{H}), 8.38(\mathrm{t}, J=1.6 \mathrm{~Hz}, 1 \mathrm{H}), 8.17(\mathrm{ddd}, J=9.0,2.4,1.4 \mathrm{~Hz}, 1 \mathrm{H}), 7.30(\mathrm{dt}, J=8.0,2.2$ $\mathrm{Hz}, 1 \mathrm{H}) ;{ }^{13} \mathrm{C}$ NMR (101 MHz, Chloroform- $\left.d\right) \delta 164.88(\mathrm{~d}, J=3.5 \mathrm{~Hz}), 163.15(\mathrm{~d}, J=251.2 \mathrm{~Hz})$, 158.22, 136.59 (d, $J=10.2 \mathrm{~Hz}), 134.68$ (d, $J=9.2 \mathrm{~Hz}), 124.27$ (d, $J=3.3 \mathrm{~Hz}), 120.72$ (d, $J=24.7$ $\mathrm{Hz}), 113.64(\mathrm{~d}, J=24.2 \mathrm{~Hz})$; MS (ESI) m/z [M+ H] $: 211.04$.

\section{3-(6-Chloro-3-fluorophenyl)-1,2,4,5-tetrazine (5c)}

The compound was obtained from 3-fluoro-5-chlorobenzonitrile (0.62 g, $4.00 \mathrm{mmol})$ following the procedure employed for 2a. Purification by flash chromatography (95/5 Heptane/EtOAc) afforded 
$0.14 \mathrm{~g}(17 \%)$ of $5 \mathbf{c}$ as a red solid. $\mathrm{Rf}=0.48\left(\right.$ Heptane/EtOAc 80/20); ${ }^{1} \mathrm{H}$ NMR $(600 \mathrm{MHz}$, Chloroformd) $\delta 10.35(\mathrm{~s}, 1 \mathrm{H}), 7.76(\mathrm{dd}, J=8.4,3.0 \mathrm{~Hz}, 1 \mathrm{H}), 7.62(\mathrm{dd}, J=8.9,4.8 \mathrm{~Hz}, 1 \mathrm{H}), 7.31$ (ddd, $J=9.1$, 7.5, 3.1 Hz, 1H); ${ }^{13} \mathrm{C}$ NMR (151 MHz, Chloroform- $d$ ) $\delta 167.66(\mathrm{~d}, J=2.3 \mathrm{~Hz}), 161.24(\mathrm{~d}, J=249.1$ Hz), 157.29 (d, $J=2.9 \mathrm{~Hz}), 132.88$ (d, $J=8.1 \mathrm{~Hz}), 132.74$ (d, $J=8.0 \mathrm{~Hz}), 128.84$ (d, $J=3.6 \mathrm{~Hz})$, $119.94(\mathrm{~d}, J=22.5 \mathrm{~Hz}), 119.11(\mathrm{~d}, J=25.1 \mathrm{~Hz}) ; \mathrm{MS}(\mathrm{ESI}) \mathrm{m} / \mathrm{z}[\mathrm{M}+\mathrm{H}]^{+}: 211.03$.

\section{3-(4-Bromo-3-fluorophenyl)-1,2,4,5-tetrazine (6a)}

The compound was obtained from 3-fluoro-4-bromobenzonitrile (0.80 g, $4.00 \mathrm{mmol})$ following the procedure employed for 2a. Purification by flash chromatography (95/5 Heptane/EtOAc) afforded $0.20 \mathrm{~g}(20 \%)$ of $6 \mathbf{a}$ as a red solid. $\mathrm{Rf}=0.42$ (Heptane/EtOAc 80/20); ${ }^{1} \mathrm{H} \mathrm{NMR}(400 \mathrm{MHz}$, Chloroformd) $\delta 10.28(\mathrm{~s}, 1 \mathrm{H}), 8.49-8.37(\mathrm{~m}, 2 \mathrm{H}), 7.67(\mathrm{dd}, J=8.6,7.3 \mathrm{~Hz}, 1 \mathrm{H}) ;{ }^{13} \mathrm{C} \mathrm{NMR}(101 \mathrm{MHz}$, Chloroform- $d$ ) $\delta 165.14(\mathrm{~d}, J=3.0 \mathrm{~Hz}), 158.72(\mathrm{~d}, J=250.5 \mathrm{~Hz}), 157.99,131.98(\mathrm{~d}, J=7.3 \mathrm{~Hz})$, 131.78, 126.67 (d, $J=17.7 \mathrm{~Hz}), 124.53(\mathrm{~d}, J=3.8 \mathrm{~Hz}), 116.17$ (d, $J=23.7 \mathrm{~Hz})$; MS (ESI) m/z [M + $\mathrm{H}]^{+}: 254.98$.

\section{3-(5-Bromo-3-fluorophenyl)-1,2,4,5-tetrazine (6b)}

The compound was obtained from 3-fluoro-5-bromobenzonitrile (0.80 g, $4.00 \mathrm{mmol})$ following the procedure employed for 2a. Purification by flash chromatography (95/5 Heptane/EtOAc) afforded $0.20 \mathrm{~g}(20 \%)$ of $\mathbf{6 b}$ as a red solid. $\mathrm{Rf}=0.41$ (Heptane/EtOAc 80/20); ${ }^{1} \mathrm{H}$ NMR (400 MHz, Chloroformd) $\delta 10.21(\mathrm{~s}, 1 \mathrm{H}), 8.52(\mathrm{~d}, J=1.6 \mathrm{~Hz}, 1 \mathrm{H}), 8.21(\mathrm{ddd}, J=9.1,2.5,1.5 \mathrm{~Hz}, 1 \mathrm{H}), 7.45(\mathrm{ddd}, J=7.8$, 2.4, $1.8 \mathrm{~Hz}, 1 \mathrm{H}) ;{ }^{13} \mathrm{C}$ NMR (101 MHz, Chloroform- $d$ ) $\delta 164.74(\mathrm{~d}, J=3.3 \mathrm{~Hz}), 163.06$ (d, $J=252.3$ $\mathrm{Hz}), 158.21,134.93$ (d, $J=8.9 \mathrm{~Hz}), 127.16$ (d, $J=3.3 \mathrm{~Hz}), 123.88$ (d, $J=9.5 \mathrm{~Hz}), 123.59(\mathrm{~d}, J=$ $24.5 \mathrm{~Hz}), 114.09$ (d, $J=24.0 \mathrm{~Hz})$; MS (ESI) m/z [M+ H] $]^{+} 254.99$.

\section{3-(6-Bromo-3-fluorophenyl)-1,2,4,5-tetrazine (6c)}

The compound was obtained from 3-fluoro-6-bromobenzonitrile $(0.40 \mathrm{~g}, 2.00 \mathrm{mmol})$ following the procedure employed for 2a. Purification by flash chromatography (95/5 Heptane/EtOAc) afforded $0.15 \mathrm{~g}(29 \%)$ of $\mathbf{6 c}$ as a red solid. $\mathrm{Rf}=0.45$ (Heptane/EtOAc 80/20); ${ }^{1} \mathrm{H}$ NMR (400 MHz, Chloroformd) $\delta 10.34(\mathrm{~s}, 1 \mathrm{H}), 7.78(\mathrm{dd}, J=8.9,5.1 \mathrm{~Hz}, 1 \mathrm{H}), 7.70(\mathrm{dd}, J=8.5,3.1 \mathrm{~Hz}, 1 \mathrm{H}), 7.22(\mathrm{ddd}, J=8.9$, 7.6, 3.1 Hz, 1H); ${ }^{13} \mathrm{C}$ NMR (101 MHz, Chloroform- $d$ ) $\delta 168.38,161.88$ (d, $\left.J=249.5 \mathrm{~Hz}\right), 157.31$, 135.95 (d, $J=7.8 \mathrm{~Hz}), 134.91$ (d, $J=8.0 \mathrm{~Hz}), 120.06$ (d, $J=22.1 \mathrm{~Hz}), 119.34$ (d, $J=24.9 \mathrm{~Hz}), 116.68$ $(\mathrm{d}, J=3.6 \mathrm{~Hz}) ; \mathrm{MS}(\mathrm{ESI}) \mathrm{m} / \mathrm{z}[\mathrm{M}+\mathrm{H}]^{+}: 255.01$. 


\section{3-(4-Iodo-3-fluorophenyl)-1,2,4,5-tetrazine (7a)}

The compound was obtained from 3-fluoro-4-iodobenzonitrile $(0.99 \mathrm{~g}, 4.00 \mathrm{mmol})$ following the procedure employed for 2a. Purification by flash chromatography (95/5 Heptane/EtOAc) afforded $0.33 \mathrm{~g}(27 \%)$ of $7 \mathbf{a}$ as a red solid. Rf $=0.39$ (Heptane/EtOAc 80/20); ${ }^{1} \mathrm{H}$ NMR (400 MHz, Chloroform- $d$ ) $\delta 10.19$ (s, 1H), 8.19 (dd, $J=8.7,2.0 \mathrm{~Hz}, 1 \mathrm{H}), 8.07$ (dd, $J=8.3,1.9 \mathrm{~Hz}, 1 \mathrm{H}), 7.92$ $(\mathrm{dd}, J=8.3,6.2 \mathrm{~Hz}, 1 \mathrm{H}) ;{ }^{13} \mathrm{C}$ NMR $(101 \mathrm{MHz}$, Chloroform- $d$ ) $\delta 164.29$ (d, $J=2.9 \mathrm{~Hz}), 161.40$ (d, $J$ $=246.8 \mathrm{~Hz}), 157.04,139.61(\mathrm{~d}, J=1.9 \mathrm{~Hz}), 132.83(\mathrm{~d}, J=7.6 \mathrm{~Hz}), 123.98(\mathrm{~d}, J=3.6 \mathrm{~Hz}), 113.81$ (d, $J=26.8 \mathrm{~Hz}), 87.16(\mathrm{~d}, J=25.7 \mathrm{~Hz})$; MS (ESI) $\mathrm{m} / \mathrm{z}[\mathrm{M}+\mathrm{H}]^{+}: 302.99$.

\section{3-(5-Iodo-3-fluorophenyl)-1,2,4,5-tetrazine (7b)}

The compound was obtained from 3-fluoro-5-iodobenzonitrile $(0.99 \mathrm{~g}, 4.00 \mathrm{mmol})$ following the procedure employed for 2a. Purification by flash chromatography (95/5 Heptane/EtOAc) afforded $0.31 \mathrm{~g}(26 \%)$ of $\mathbf{7 b}$ as a red solid. $\mathrm{Rf}=0.39$ (Heptane/EtOAc 80/20); ${ }^{1} \mathrm{H}$ NMR (400 MHz, Chloroform- $d$ ) $\delta 10.21(\mathrm{~s}, 1 \mathrm{H}), 8.72(\mathrm{~d}, J=1.4 \mathrm{~Hz}, 1 \mathrm{H}), 8.23(\mathrm{dt}, J=9.1,1.9 \mathrm{~Hz}, 1 \mathrm{H}), 7.65(\mathrm{dt}, J=$ 7.6, $1.9 \mathrm{~Hz}, 1 \mathrm{H}) ;{ }^{13} \mathrm{C}$ NMR $(101 \mathrm{MHz}$, Chloroform- $d$ ) $\delta 164.51(\mathrm{~d}, J=3.2 \mathrm{~Hz}), 162.68(\mathrm{~d}, J=253.2$ $\mathrm{Hz}), 158.18,134.97(\mathrm{~d}, J=8.5 \mathrm{~Hz}), 133.04$ (d, $J=3.3 \mathrm{~Hz}), 129.29$ (d, $J=23.7 \mathrm{~Hz}), 114.77(\mathrm{~d}, J=$ $24.1 \mathrm{~Hz}), 94.36(\mathrm{~d}, J=8.0 \mathrm{~Hz})$; MS (ESI) $\mathrm{m} / \mathrm{z}[\mathrm{M}+\mathrm{H}]^{+}: 302.98$.

\section{3-(6-Iodo-3-fluorophenyl)-1,2,4,5-tetrazine (7c)}

The compound was obtained from 3-fluoro-6-iodobenzonitrile (0.99 g, $4.00 \mathrm{mmol})$ following the procedure employed for 2a. Purification by flash chromatography (95/5 Heptane/EtOAc) afforded $0.25 \mathrm{~g}(21 \%)$ of $7 \mathbf{c}$ as a red solid. $\mathrm{Rf}=0.37$ (Heptane/EtOAc 80/20); ${ }^{1} \mathrm{H}$ NMR $(400 \mathrm{MHz}$, Chloroformd) $\delta 10.26(\mathrm{~s}, 1 \mathrm{H}), 7.99(\mathrm{dd}, J=8.8,5.3 \mathrm{~Hz}, 1 \mathrm{H}), 7.64(\mathrm{dd}, J=8.8,3.0 \mathrm{~Hz}, 1 \mathrm{H}), 7.00$ (ddd, $J=8.7$, 7.8, 3.0 Hz, 1H); ${ }^{13} \mathrm{C}$ NMR (101 MHz, Chloroform- $d$ ) $\delta 169.14(\mathrm{~d}, J=2.5 \mathrm{~Hz}), 162.94(\mathrm{~d}, J=250.3$ $\mathrm{Hz}), 157.34,142.62(\mathrm{~d}, J=7.5 \mathrm{~Hz}), 138.35$ (d, $J=7.7 \mathrm{~Hz}), 120.15(\mathrm{~d}, J=21.6 \mathrm{~Hz}), 119.01(\mathrm{~d}, J=$ $24.5 \mathrm{~Hz}), 88.53(\mathrm{~d}, J=3.7 \mathrm{~Hz})$; MS (ESI) $\mathrm{m} / \mathrm{z}[\mathrm{M}+\mathrm{H}]^{+}: 302.99$.

\section{2-Fluoro-4-(1,2,4,5-tetrazin-3-yl)phenol (8a)}

The compound was obtained from 3-fluoro-4-hydroxybenzonitrile $(0.55 \mathrm{~g}, 4.00 \mathrm{mmol})$ following the procedure employed for 2a. Purification by flash chromatography (80/20 Heptane/EtOAc) afforded $0.15 \mathrm{~g}(19 \%)$ of $8 \mathrm{a}$ as a red solid. $\mathrm{Rf}=0.26$ (Heptane/EtOAc 80/20); ${ }^{1} \mathrm{H}$ NMR (400 MHz, Methanol$\left.d_{4}\right) \delta 10.24(\mathrm{~s}, 1 \mathrm{H}), 8.40-8.12(\mathrm{~m}, 2 \mathrm{H}), 7.14(\mathrm{t}, J=8.7 \mathrm{~Hz}, 1 \mathrm{H}) ;{ }^{13} \mathrm{C}$ NMR $\left(101 \mathrm{MHz}\right.$, Methanol- $\left.d_{4}\right)$ $\delta 165.50,157.39,151.85(\mathrm{~d}, J=241.9 \mathrm{~Hz}), 149.87$ (d, $J=12.9 \mathrm{~Hz}), 124.85$ (d, $J=3.1 \mathrm{~Hz}), 123.47$ $(\mathrm{d}, J=6.7 \mathrm{~Hz}), 118.09$ (d, $J=3.1 \mathrm{~Hz}), 115.20(\mathrm{~d}, J=21.1 \mathrm{~Hz}) ; \mathrm{MS}(\mathrm{ESI}) \mathrm{m} / \mathrm{z}[\mathrm{M}+\mathrm{H}]^{+}: 193.07$. 


\section{3-Fluoro-5-(1,2,4,5-tetrazin-3-yl)phenol (8b)}

The compound was obtained from 3-fluoro-5-hydroxybenzonitrile $(0.55 \mathrm{~g}, 4.00 \mathrm{mmol})$ following the procedure employed for 2a. Purification by flash chromatography (80/20 Heptane/EtOAc) afforded $0.23 \mathrm{~g}(30 \%)$ of $\mathbf{8 b}$ as a red solid. Rf $=0.28$ (Heptane/EtOAc 80/20); ${ }^{1} \mathrm{H}$ NMR (400 MHz, DMSO$\left.d_{6}\right) \delta 10.62(\mathrm{~s}, 1 \mathrm{H}), 10.50(\mathrm{~s}, 1 \mathrm{H}), 7.79(\mathrm{t}, J=1.8 \mathrm{~Hz}, 1 \mathrm{H}), 7.67(\mathrm{dt}, J=9.7,1.9 \mathrm{~Hz}, 1 \mathrm{H}), 6.91(\mathrm{dd}, J$ $=10.6,2.3 \mathrm{~Hz}, 1 \mathrm{H}) ;{ }^{13} \mathrm{C}$ NMR $\left(101 \mathrm{MHz}, \mathrm{DMSO}-d_{6}\right) \delta 165.12(\mathrm{~d}, J=3.9 \mathrm{~Hz}), 163.96(\mathrm{~d}, J=243.1$ $\mathrm{Hz}), 160.36(\mathrm{~d}, J=12.2 \mathrm{~Hz}), 158.77,134.88$ (d, $J=10.9 \mathrm{~Hz}), 111.30$ (d, $J=2.4 \mathrm{~Hz}), 107.31(\mathrm{~d}, J=$ $23.8 \mathrm{~Hz}), 105.34$ (d, $J=24.4 \mathrm{~Hz})$; MS (ESI) m/z [M + H] $]^{+}$193.06.

\section{4-Fluoro-2-(1,2,4,5-tetrazin-3-yl)phenol (8c)}

The compound was obtained from 3-fluoro-6-hydroxybenzonitrile $(0.55 \mathrm{~g}, 4.00 \mathrm{mmol})$ following the procedure employed for 2a. Purification by flash chromatography (80/20 Heptane/EtOAc) afforded $0.18 \mathrm{~g}(23 \%)$ of $\mathbf{8 c}$ as a red solid. $\mathrm{Rf}=0.37$ (Heptane/EtOAc 80/20); ${ }^{1} \mathrm{H}$ NMR (400 MHz, Chloroformd) $\delta 10.89(\mathrm{~s}, 1 \mathrm{H}), 10.28(\mathrm{~s}, 1 \mathrm{H}), 8.33(\mathrm{dd}, J=9.3,3.2 \mathrm{~Hz}, 1 \mathrm{H}), 7.28$ (ddd, $J=9.2,7.4,3.2 \mathrm{~Hz}, 1 \mathrm{H})$, $7.10(\mathrm{dd}, J=9.2,4.6 \mathrm{~Hz}, 1 \mathrm{H}) ;{ }^{13} \mathrm{C}$ NMR (101 MHz, Chloroform- $d$ ) $\delta 166.76$ (d, $\left.J=3.1 \mathrm{~Hz}\right), 157.06$, $156.72(\mathrm{~d}, J=1.8 \mathrm{~Hz}), 156.30(\mathrm{~d}, J=238.9 \mathrm{~Hz}), 123.33(\mathrm{~d}, J=23.9 \mathrm{~Hz}), 120.32(\mathrm{~d}, J=7.7 \mathrm{~Hz})$, $114.02(\mathrm{~d}, J=8.3 \mathrm{~Hz}), 113.87$ (d, $J=25.6 \mathrm{~Hz})$; MS (ESI) m/z [M + H] $]^{+} 193.08$.

\section{3-(3-Fluoro-4-methoxyphenyl)-1,2,4,5-tetrazine (9a)}

The compound was obtained from 3-fluoro-4-methoxybenzonitrile $(0.60 \mathrm{~g}, 4.00 \mathrm{mmol})$ following the procedure employed for 2a. Purification by flash chromatography (95/5 Heptane/EtOAc) afforded $0.24 \mathrm{~g}(29 \%)$ of $9 \mathrm{a}$ as a red solid. $\mathrm{Rf}=0.39$ (Heptane/EtOAc 80/20); ${ }^{1} \mathrm{H}$ NMR (400 MHz, Chloroformd) $\delta 10.18(\mathrm{~s}, 1 \mathrm{H}), 8.45(\mathrm{ddd}, J=8.6,2.1,1.3 \mathrm{~Hz}, 1 \mathrm{H}), 8.38(\mathrm{dd}, J=12.1,2.2 \mathrm{~Hz}, 1 \mathrm{H}), 7.18(\mathrm{t}, J=$ $8.5 \mathrm{~Hz}, 1 \mathrm{H}), 4.04$ (s, 3H); ${ }^{13} \mathrm{C}$ NMR (101 MHz, Chloroform- $d$ ) $\delta 165.53,157.51,152.72$ (d, J=247.7 $\mathrm{Hz}), 152.06$ (d, $J=10.7 \mathrm{~Hz}), 125.29$ (d, $J=3.6 \mathrm{~Hz}), 124.35$ (d, $J=7.2 \mathrm{~Hz}), 115.80$ (d, $J=20.8 \mathrm{~Hz})$, $113.52(\mathrm{~d}, J=2.2 \mathrm{~Hz}), 56.36$; MS (ESI) $\mathrm{m} / \mathrm{z}[\mathrm{M}+\mathrm{H}]^{+}: 207.09$.

\section{3-(3-Fluoro-5-methoxyphenyl)-1,2,4,5-tetrazine (9b)}

The compound was obtained from 3-fluoro-5-methoxybenzonitrile $(0.60 \mathrm{~g}, 4.00 \mathrm{mmol})$ following the procedure employed for 2a. Purification by flash chromatography (95/5 Heptane/EtOAc) afforded $0.21 \mathrm{~g}(26 \%)$ of $9 \mathrm{~b}$ as a red solid. $\mathrm{Rf}=0.41$ (Heptane/EtOAc 80/20); ${ }^{1} \mathrm{H}$ NMR (400 MHz, Chloroformd) $\delta 10.24(\mathrm{~s}, 1 \mathrm{H}), 7.98(\mathrm{~d}, J=2.0 \mathrm{~Hz}, 1 \mathrm{H}), 7.94(\mathrm{ddd}, J=9.1,2.4,1.4 \mathrm{~Hz}, 1 \mathrm{H}), 6.90(\mathrm{dd}, J=10.1$, $2.4 \mathrm{~Hz}, 1 \mathrm{H}), 3.93$ (s, 3H); ${ }^{13} \mathrm{C}$ NMR (101 MHz, Chloroform- $d$ ) $\delta 165.63$ (d, $\left.J=3.9 \mathrm{~Hz}\right), 164.07$ (d, $J$ 
$=246.5 \mathrm{~Hz}), 161.75(\mathrm{~d}, J=11.4 \mathrm{~Hz}), 158.02,133.96(\mathrm{~d}, J=10.7 \mathrm{~Hz}), 108.96(\mathrm{~d}, J=2.8 \mathrm{~Hz}), 107.73$ (d, $J=24.7 \mathrm{~Hz}), 106.98(\mathrm{~d}, J=24.9 \mathrm{~Hz}), 55.96$; MS (ESI) m/z [M + H] : 207.08 .

\section{3-(3-Fluoro-6-methoxyphenyl)-1,2,4,5-tetrazine (9c)}

The compound was obtained from 3-fluoro-6-methoxybenzonitrile $(0.60 \mathrm{~g}, 4.00 \mathrm{mmol})$ following the procedure employed for 2a. Purification by flash chromatography (95/5 Heptane/EtOAc) afforded $0.13 \mathrm{~g}(16 \%)$ of $9 \mathrm{c}$ as a red solid. $\mathrm{Rf}=0.18\left(\right.$ Heptane/EtOAc 90/10); ${ }^{1} \mathrm{H}$ NMR $(600 \mathrm{MHz}$, Chloroformd) $\delta 10.23(\mathrm{~s}, 1 \mathrm{H}), 8.26(\mathrm{~s}, 1 \mathrm{H}), 8.12(\mathrm{~d}, J=9.4 \mathrm{~Hz}, 1 \mathrm{H}), 7.17(\mathrm{~d}, J=9.1 \mathrm{~Hz}, 1 \mathrm{H}), 2.50(\mathrm{~s}, 3 \mathrm{H}) ;{ }^{13} \mathrm{C}$ NMR (151 MHz, Chloroform- $d$ ) $\delta 165.99$ (d, $J=3.4$ Hz), 164.27 (d, $J=247.1 \mathrm{~Hz}), 158.11,141.97$ (d, $J=7.8 \mathrm{~Hz}), 133.45(\mathrm{~d}, J=8.9 \mathrm{~Hz}), 124.81,120.95$ (d, $J=21.2 \mathrm{~Hz}), 112.44$ (d, $J=24.2 \mathrm{~Hz})$, 21.60; MS (ESI) m/z [M+ H] : 207.09.

\section{2-Fluoro-4-(1,2,4,5-tetrazin-3-yl)benzoic acid (10a)}

The compound was obtained from 4-cyano-2-fluorobenzoic acid ( $0.66 \mathrm{~g}, 4.00 \mathrm{mmol})$ following the procedure employed for 2a. Purification by flash chromatography $\left(98 / 2 \mathrm{CH}_{2} \mathrm{Cl}_{2} / \mathrm{MeOH}\right)$ afforded $0.32 \mathrm{~g}(36 \%)$ of $10 \mathrm{a}$ as a red solid. $\mathrm{Rf}=0.31($ Heptane/EtOAc $30 / 70) ;{ }^{1} \mathrm{H}$ NMR $(600 \mathrm{MHz}$, Methanol$\left.d_{4}\right) \delta 10.44(\mathrm{~s}, 1 \mathrm{H}), 8.50(\mathrm{dd}, J=8.1,1.6 \mathrm{~Hz}, 1 \mathrm{H}), 8.40(\mathrm{dd}, J=11.5,1.7 \mathrm{~Hz}, 1 \mathrm{H}), 8.20(\mathrm{t}, J=7.7 \mathrm{~Hz}$, $1 \mathrm{H}) ;{ }^{13} \mathrm{C}$ NMR $\left(151 \mathrm{MHz}\right.$, Methanol- $\left.d_{4}\right) \delta 165.11(\mathrm{~d}, J=3.3 \mathrm{~Hz}), 165.04(\mathrm{~d}, J=2.7 \mathrm{~Hz}), 162.10(\mathrm{~d}$, $J=259.0 \mathrm{~Hz}), 158.24$ (d, $J=10.2 \mathrm{~Hz}), 137.97$ (d, $J=8.8 \mathrm{~Hz}), 132.85$ (d, $J=1.2 \mathrm{~Hz}), 123.10$ (d, $J=$ $4.0 \mathrm{~Hz}), 122.84$ (d, $J=10.7 \mathrm{~Hz}), 115.83$ (d, $J=25.7 \mathrm{~Hz})$; MS (ESI) m/z [M - H] : 219.04.

\section{3-Fluoro-5-(1,2,4,5-tetrazin-3-yl)benzoic acid (10b)}

The compound was obtained from 5-cyano-3-fluorobenzoic acid ( $0.66 \mathrm{~g}, 4.00 \mathrm{mmol})$ following the procedure employed for 2a. Purification by flash chromatography $\left(98 / 2 \mathrm{CH}_{2} \mathrm{Cl}_{2} / \mathrm{MeOH}\right)$ afforded $0.21 \mathrm{~g}(24 \%)$ of $\mathbf{1 0 b}$ as a red solid. $\mathrm{Rf}=0.33$ (Heptane/EtOAc 30/70); ${ }^{1} \mathrm{H}$ NMR $(400 \mathrm{MHz}$, Methanol$\left.d_{4}\right) \delta 10.32(\mathrm{~s}, 1 \mathrm{H}), 8.95(\mathrm{t}, J=1.5 \mathrm{~Hz}, 1 \mathrm{H}), 8.42(\mathrm{ddd}, J=9.2,2.6,1.5 \mathrm{~Hz}, 1 \mathrm{H}), 7.90(\mathrm{ddd}, J=8.8$, 2.7, $1.4 \mathrm{~Hz}, 1 \mathrm{H}) ;{ }^{13} \mathrm{C}$ NMR $\left(101 \mathrm{MHz}\right.$, Methanol- $\left.d_{4}\right) \delta 166.04,165.10,163.08(\mathrm{~d}, J=247.1 \mathrm{~Hz})$, 158.29, $134.91(\mathrm{~d}, J=8.2 \mathrm{~Hz}), 134.49$ (d, $J=7.3 \mathrm{~Hz}), 124.63$ (d, $J=3.1 \mathrm{~Hz}), 119.87$ (d, $J=23.2$ Hz), 118.29 (d, $J=24.7 \mathrm{~Hz})$; MS (ESI) m/z [M - H] : 219.06.

\section{Methyl 2-fluoro-4-(1,2,4,5-tetrazin-3-yl)benzoate (11a)}

Compound 10a $(0.20 \mathrm{~g}, 0.90 \mathrm{mmol})$ was solubilized in $\mathrm{MeOH}(30 \mathrm{~mL})$ and then a solution of $\mathrm{HCl}$ in dioxane (4M, $2.0 \mathrm{~mL}$ ) was added. The reaction as stirred for $3 \mathrm{~h}$ and then the solvent was removed under reduced pressure. The compound was purified by flash chromatography (90/10 
Heptane/EtoAc) to give $0.14 \mathrm{~g}(66 \%)$ of 11a as a red solid. $\mathrm{Rf}=0.41($ Heptane/EtOAc $80 / 20) ;{ }^{1} \mathrm{H}$ NMR (400 MHz, Chloroform- $d$ ) $\delta 10.32$ (s, 1H), 8.50 (dd, $J=8.3,1.6 \mathrm{~Hz}, 1 \mathrm{H}), 8.44$ (dd, $J=11.3$, $1.6 \mathrm{~Hz}, 1 \mathrm{H}), 8.18(\mathrm{dd}, J=8.2,7.2 \mathrm{~Hz}, 1 \mathrm{H}), 4.01(\mathrm{~s}, 3 \mathrm{H}) ;{ }^{13} \mathrm{C}$ NMR $(101 \mathrm{MHz}$, Chloroform- $d) \delta$ 165.07 (d, $J=2.8 \mathrm{~Hz}), 164.10$ (d, $J=3.8 \mathrm{~Hz}), 162.14$ (d, $J=261.3 \mathrm{~Hz}), 158.15,137.32$ (d, $J=8.9$ $\mathrm{Hz}), 133.17(\mathrm{~d}, J=1.3 \mathrm{~Hz}), 123.47$ (d, $J=4.1 \mathrm{~Hz}), 122.51,116.72$ (d, $J=25.6 \mathrm{~Hz}), 52.73$; MS (ESI) $\mathrm{m} / \mathrm{z}[\mathrm{M}+\mathrm{H}]^{+}: 235.08$.

\section{Methyl 3-fluoro-5-(1,2,4,5-tetrazin-3-yl)benzoate (11b)}

Compound 10b $(0.20 \mathrm{~g}, 0.90 \mathrm{mmol})$ was solubilized in $\mathrm{MeOH}(30 \mathrm{~mL})$ and then a solution of $\mathrm{HCl}$ in dioxane (4M, $2.0 \mathrm{~mL})$ was added. The reaction as stirred for $3 \mathrm{~h}$ and then the solvent was removed under reduced pressure. The compound was purified by flash chromatography (90/10 Heptane/EtoAc) to give $0.18 \mathrm{~g}(85 \%)$ of $\mathbf{1 1 b}$ as a red solid. $\mathrm{Rf}=0.43($ Heptane/EtOAc $80 / 20) ;{ }^{1} \mathrm{H}$ NMR (400 MHz, Chloroform- $d$ ) $\delta 10.31$ (s, 1H), 9.13 (t, $J=1.5 \mathrm{~Hz}, 1 \mathrm{H}), 8.54$ (ddd, $J=9.0,2.6,1.5$ $\mathrm{Hz}, 1 \mathrm{H}), 8.02$ (ddd, $J=8.5,2.6,1.4 \mathrm{~Hz}, 1 \mathrm{H}), 4.00$ (s, 3H); ${ }^{13} \mathrm{C}$ NMR (101 MHz, Chloroform- $d$ ) $\delta$ $165.17(\mathrm{~d}, J=3.2 \mathrm{~Hz}), 165.04(\mathrm{~d}, J=3.1 \mathrm{~Hz}), 163.11$ (d, $J=249.1 \mathrm{~Hz}), 158.22,134.11(\mathrm{~d}, J=8.1$ $\mathrm{Hz}), 133.72$ (d, $J=7.5 \mathrm{~Hz}), 125.12(\mathrm{~d}, J=3.1 \mathrm{~Hz}), 121.01$ (d, $J=23.3 \mathrm{~Hz}), 119.27$ (d, $J=24.2 \mathrm{~Hz})$, 52.78; MS (ESI) m/z [M+ H] $]^{+}: 235.07$.

\section{Tert-butyl 2-fluoro-4-(1,2,4,5-tetrazin-3-yl)benzoate (12a)}

\section{Tert-butyl 4-cyano-2-fluorobenzoate (21a)}

The compound was obtained following the literature procedure. ${ }^{[13]}$ 4-Cyano-2-fluorobenzoic acid (1.09 g, $6.54 \mathrm{mmol})$ was dissolved in $t$-BuOH $(9 \mathrm{~mL})$ and THF $(3 \mathrm{~mL})$. Boc anhydride $(2.90 \mathrm{~g}, 13.27$ mmol) was added followed by DMAP $(0.24 \mathrm{~g}, 1.99 \mathrm{mmol})$. The mixture was stirred at rt under $\mathrm{N}_{2}$ for $12 \mathrm{~h}$. The solvents were removed. The residue was dissolved in EtOAc $(30 \mathrm{~mL})$ and washed with saturated aqueous $\mathrm{NaHCO}_{3}(2 \times 30 \mathrm{~mL})$ and brine $(2 \times 30 \mathrm{~mL})$. It was dried over anhydrous $\mathrm{MgSO}_{4}$ and concentrated under reduced pressure to give $1.25 \mathrm{~g}$ (85\%) of the desired compound as white solid. $\mathrm{Rf}=0.30$ (Heptane/EtOAc 80/20); ${ }^{1} \mathrm{H}$ NMR $(600 \mathrm{MHz}$, Chloroform- $d) \delta 7.98(\mathrm{dd}, J=$ 8.0, 7.1 Hz, 1H), $7.50(\mathrm{dd}, J=8.1,1.5 \mathrm{~Hz}, 1 \mathrm{H}), 7.43(\mathrm{dd}, J=9.7,1.5 \mathrm{~Hz}, 1 \mathrm{H}), 1.62(\mathrm{~s}, 9 \mathrm{H}) ;{ }^{13} \mathrm{C} \mathrm{NMR}$ $(151 \mathrm{MHz}$, Chloroform- $d$ ) $\delta 161.93$ (d, $J=3.8 \mathrm{~Hz}), 161.02(\mathrm{~d}, J=262.6 \mathrm{~Hz}), 132.88(\mathrm{~d}, J=1.9 \mathrm{~Hz})$, 127.55 (d, $J=4.6 \mathrm{~Hz}), 125.14$ (d, $J=10.5 \mathrm{~Hz}), 120.76$ (d, $J=26.3 \mathrm{~Hz}), 116.85$ (d, $J=9.5 \mathrm{~Hz}), 116.79$ $(\mathrm{d}, J=2.7 \mathrm{~Hz}), 83.36,28.09$.

Tert-butyl 2-fluoro-4-(1,2,4,5-tetrazin-3-yl)benzoate

The compound was obtained from tert-butyl 4-cyano-2-fluorobenzoate (1.19 g, $5.38 \mathrm{mmol}$ ) following the procedure employed for $\mathbf{2 a}$. Purification by flash chromatography (95/5 Heptane/EtOAc) afforded 
$0.21 \mathrm{~g}(14 \%)$ of 12a as a red solid. Rf $=0.41$ (Heptane/EtOAc 80/20); ${ }^{1} \mathrm{H}$ NMR (400 MHz, Chloroform- $d$ ) $\delta 10.29(\mathrm{~s}, 1 \mathrm{H}), 8.46(\mathrm{dd}, J=8.2,1.6 \mathrm{~Hz}, 1 \mathrm{H}), 8.39(\mathrm{dd}, J=11.3,1.6 \mathrm{~Hz}, 1 \mathrm{H}), 8.08$ $(\mathrm{dd}, J=8.1,7.2 \mathrm{~Hz}, 1 \mathrm{H}), 1.64(\mathrm{~s}, 9 \mathrm{H}),{ }^{13} \mathrm{C}$ NMR $(101 \mathrm{MHz}$, Chloroform- $d$ ) $\delta 165.19(\mathrm{~d}, J=2.7 \mathrm{~Hz})$, $162.75(\mathrm{~d}, J=3.7 \mathrm{~Hz}), 162.04$ (d, $J=260.2 \mathrm{~Hz}), 158.10,136.64$ (d, $J=8.8 \mathrm{~Hz}), 132.93,124.65$ (d, $J$ $=10.5 \mathrm{~Hz}), 123.34(\mathrm{~d}, J=4.0 \mathrm{~Hz}), 116.65(\mathrm{~d}, J=25.8 \mathrm{~Hz}), 82.84,28.19 ;$ MS (ESI) m/z [M + H] $]^{+}$ 291.14.

\section{Tert-butyl 3-fluoro-5-(1,2,4,5-tetrazin-3-yl)benzoate (12b)}

Tert-butyl 5-cyano-3-fluorobenzoate (21b)

The compound was obtained following the literature procedure. ${ }^{[13]} 5$-Cyano-3-fluorobenzoic acid $(1.09 \mathrm{~g}, 6.54 \mathrm{mmol})$ was dissolved in $t$-BuOH $(9 \mathrm{~mL})$ and THF $(3 \mathrm{~mL})$. Boc anhydride $(2.90 \mathrm{~g}, 13.27$ mmol) was added followed by DMAP $(0.24 \mathrm{~g}, 1.99 \mathrm{mmol})$. The mixture was stirred at $\mathrm{rt}$ under $\mathrm{N}_{2}$ for $12 \mathrm{~h}$. The solvents were removed. The residue was dissolved in EtOAc $(30 \mathrm{~mL})$ and washed with saturated aqueous $\mathrm{NaHCO}_{3}(2 \times 30 \mathrm{~mL})$ and brine $(2 \times 30 \mathrm{~mL})$. It was dried over anhydrous $\mathrm{MgSO}_{4}$ and concentrated under reduced pressure to give $1.42 \mathrm{~g}$ (97\%) of the desired compound as white solid. Rf = $0.32\left(\right.$ Heptane/EtOAc 80/20); ${ }^{1} \mathrm{H}$ NMR $(400 \mathrm{MHz}$, Chloroform- $d$ ) $\delta 8.05(\mathrm{dt}, J=$ 4.2, $1.4 \mathrm{~Hz}, 1 \mathrm{H}), 7.93-7.85(\mathrm{~m}, 1 \mathrm{H}), 7.54-7.45(\mathrm{~m}, 1 \mathrm{H}), 1.59(\mathrm{~s}, 9 \mathrm{H}) ;{ }^{13} \mathrm{C}$ NMR $(101 \mathrm{MHz}$, Chloroform- $d$ ) $\delta 162.40(\mathrm{~d}, J=3.0 \mathrm{~Hz}), 162.07$ (d, $J=251.6 \mathrm{~Hz}), 135.79$ (d, $J=7.3 \mathrm{~Hz}), 129.08$ (d, $J=3.5 \mathrm{~Hz}), 122.49$ (d, $J=25.1 \mathrm{~Hz}), 121.18(\mathrm{~d}, J=22.8 \mathrm{~Hz}), 116.86(\mathrm{~d}, J=3.0 \mathrm{~Hz}), 114.01(\mathrm{~d}, J=$ $9.1 \mathrm{~Hz}), 83.06,28.01$.

Tert-butyl 3-fluoro-5-(1,2,4,5-tetrazin-3-yl)benzoate

The compound was obtained from tert-butyl 5-cyano-3-fluorobenzoate (1.22 g, $5.51 \mathrm{mmol})$ following the procedure employed for $\mathbf{2 a}$. Purification by flash chromatography (95/5 Heptane/EtOAc) afforded $0.25 \mathrm{~g}(16 \%)$ of $\mathbf{1 2 b}$ as a red solid. $\mathrm{Rf}=0.37$ (Heptane/EtOAc 80/20); ${ }^{1} \mathrm{H}$ NMR (400 MHz, Chloroform- $d$ ) $\delta 10.32(\mathrm{~s}, 1 \mathrm{H}), 9.04(\mathrm{t}, J=1.5 \mathrm{~Hz}, 1 \mathrm{H}), 8.50(\mathrm{ddd}, J=9.0,2.7,1.6 \mathrm{~Hz}, 1 \mathrm{H}), 7.97$ (ddd, $J=8.6,2.7,1.4 \mathrm{~Hz}, 1 \mathrm{H}), 1.66(\mathrm{~s}, 9 \mathrm{H}) ;{ }^{13} \mathrm{C}$ NMR $(101 \mathrm{MHz}$, Chloroform- $d$ ) $\delta 165.31(\mathrm{~d}, J=3.1$ $\mathrm{Hz}), 163.58$ (d, $J=2.9 \mathrm{~Hz}), 163.05$ (d, $J=248.6 \mathrm{~Hz}), 158.15,135.70$ (d, $J=7.3 \mathrm{~Hz}), 133.84(\mathrm{~d}, J=$ $8.0 \mathrm{~Hz}), 124.92(\mathrm{~d}, J=3.1 \mathrm{~Hz}), 120.87$ (d, $J=23.1 \mathrm{~Hz}), 118.74$ (d, $J=24.4 \mathrm{~Hz}), 82.51,28.14$; MS (ESI) $\mathrm{m} / \mathrm{z}[\mathrm{M}+\mathrm{H}]^{+}: 291.16$.

\section{Tert-butyl 2-cyano-5-fluorobenzoate (21c)}

The compound was obtained following the literature procedure. ${ }^{[13]}$ 2-Cyano-4-fluorobenzoic acid $(1.09 \mathrm{~g}, 6.54 \mathrm{mmol})$ was dissolved in $t$-BuOH $(9 \mathrm{~mL})$ and THF $(3 \mathrm{~mL})$. Boc anhydride $(2.90 \mathrm{~g}, 13.27$ mmol) was added followed by DMAP $(0.24 \mathrm{~g}, 1.99 \mathrm{mmol})$. The mixture was stirred at $\mathrm{rt}$ under $\mathrm{N}_{2}$ for 
$12 \mathrm{~h}$. The solvents were removed. The residue was dissolved in EtOAc $(30 \mathrm{~mL})$ and washed with saturated aqueous $\mathrm{NaHCO}_{3}(2 \times 30 \mathrm{~mL})$ and brine $(2 \times 30 \mathrm{~mL})$. It was dried over anhydrous $\mathrm{MgSO}_{4}$ and concentrated under reduced pressure to give $1.10 \mathrm{~g}(75 \%)$ of the desired compound as white solid. $\mathrm{Rf}=0.354\left(\right.$ Heptane/EtOAc 80/20); ${ }^{1} \mathrm{H}$ NMR $(400 \mathrm{MHz}$, Chloroform- $d) \delta 8.04(\mathrm{dd}, J=$ 8.8, $5.5 \mathrm{~Hz}, 1 \mathrm{H}), 7.37(\mathrm{dd}, J=8.1,2.7 \mathrm{~Hz}, 1 \mathrm{H}), 7.28(\mathrm{ddd}, J=8.8,7.7,2.6 \mathrm{~Hz}, 1 \mathrm{H}), 1.55(\mathrm{~s}, 9 \mathrm{H}) ;{ }^{13} \mathrm{C}$ NMR (101 MHz, Chloroform-d) $\delta 163.95$ (d, $J=256.4 \mathrm{~Hz}), 162.13,130.53$ (d, $J=3.6 \mathrm{~Hz}), 121.50$ $(\mathrm{d}, J=25.1 \mathrm{~Hz}), 119.78(\mathrm{~d}, J=21.2 \mathrm{~Hz}), 116.46(\mathrm{~d}, J=2.6 \mathrm{~Hz}), 114.76(\mathrm{~d}, J=9.9 \mathrm{~Hz}), 83.90$.

\section{2-Fluoro-4-(1,2,4,5-tetrazin-3-yl)benzamide (13a)}

\section{4-Cyano-2-fluorobenzamide (22a)}

To a solution of 4-cyano-2-fluorobenzoic acid (0.99 g, $6.0 \mathrm{mmol})$ in acetonitrile $(20 \mathrm{ml})$ was added 1,1'-carbonyldiimidazole $(1.46 \mathrm{~g}, 9.0 \mathrm{mmol})$. The mixture was stirred at room temperature for $45 \mathrm{~min}$, before addition of aqueous ammonium hydroxide solution $(35 \%, 20 \mathrm{ml})$. The reaction mixture was stirred for $45 \mathrm{~min}$ and ice-cold water $(20 \mathrm{ml})$ was added. The precipitate was collected by filtration and dried to give $0.78 \mathrm{~g}(79 \%)$ of the desired compound as a white solid. $\mathrm{Rf}=0.28$ (Heptane/EtOAc 30/70); ${ }^{1} \mathrm{H}$ NMR (400 MHz, DMSO-d $\left.d_{6}\right) \delta 8.13-7.92(\mathrm{~m}, 2 \mathrm{H}), 7.86(\mathrm{~s}, 0 \mathrm{H}), 7.84-7.68(\mathrm{~m}, 2 \mathrm{H}) ;{ }^{13} \mathrm{C}$ NMR (101 MHz, DMSO-d6) $\delta 164.56,158.89$ (d, $J=251.4 \mathrm{~Hz}), 131.56$ (d, $J=4.0 \mathrm{~Hz}), 129.60(\mathrm{~d}, J$ $=15.7 \mathrm{~Hz}), 129.16(\mathrm{~d}, J=4.0 \mathrm{~Hz}), 120.79(\mathrm{~d}, J=26.7 \mathrm{~Hz}), 117.68(\mathrm{~d}, J=2.8 \mathrm{~Hz}), 114.58(\mathrm{~d}, J=$ $10.0 \mathrm{~Hz})$.

\section{2-Fluoro-4-(1,2,4,5-tetrazin-3-yl)benzamide}

The compound was obtained from 4-cyano-2-fluorobenzamide $(0.78 \mathrm{~g}, 4.75 \mathrm{mmol})$ following the procedure employed for 2a. Purification by flash chromatography $\left(98 / 2 \mathrm{CH}_{2} \mathrm{Cl}_{2} / \mathrm{MeOH}\right)$ afforded $0.21 \mathrm{~g}(20 \%)$ of $13 \mathrm{a}$ as a red solid. $\mathrm{Rf}=0.32($ Heptane/EtOAc $30 / 70) ;{ }^{1} \mathrm{H}$ NMR (400 MHz, DMSO$\left.d_{6}\right) \delta 10.68(\mathrm{~s}, 1 \mathrm{H}), 8.39(\mathrm{dd}, J=8.0,1.6 \mathrm{~Hz}, 1 \mathrm{H}), 8.30(\mathrm{dd}, J=11.1,1.6 \mathrm{~Hz}, 1 \mathrm{H}), 7.97(\mathrm{~s}, 1 \mathrm{H}), 7.93$ (t, $J=7.7 \mathrm{~Hz}, 1 \mathrm{H}), 7.83(\mathrm{~s}, 1 \mathrm{H}) ;{ }^{13} \mathrm{C} \mathrm{NMR}\left(101 \mathrm{MHz}, \mathrm{DMSO}-d_{6}\right) \delta 165.07,164.84(\mathrm{~d}, J=2.9 \mathrm{~Hz})$, $159.91(\mathrm{~d}, J=250.1 \mathrm{~Hz}), 158.83,136.15$ (d, $J=8.5 \mathrm{~Hz}), 131.81(\mathrm{~d}, J=3.4 \mathrm{~Hz}), 128.25(\mathrm{~d}, J=15.2$ $\mathrm{Hz}), 124.16$ (d, $J=3.4 \mathrm{~Hz}), 115.57$ (d, $J=25.4 \mathrm{~Hz})$; MS (ESI) m/z [M + H] $]^{+}: 220.08$.

\section{3-Fluoro-5-(1,2,4,5-tetrazin-3-yl)benzamide (13b)}

\section{5-Cyano-3-fluorobenzamide (22b)}

The compound was obtained from 5-cyano-3-fluorobenzoic acid (0.99 g, $6.0 \mathrm{mmol})$ as reported above for 22a to give $0.77 \mathrm{~g}(78 \%)$ of the desired compound as a white solid. $\mathrm{Rf}=0.30$ (Heptane/EtOAc 30/70); ${ }^{1} \mathrm{H}$ NMR (400 MHz, DMSO-d6) $\delta 8.21(\mathrm{~s}, 1 \mathrm{H}), 8.16$ (t, $J=1.5 \mathrm{~Hz}, 1 \mathrm{H}$ ), 8.05 (ddd, $J=8.4$, 2.6, $1.3 \mathrm{~Hz}, 1 \mathrm{H}), 8.01(\mathrm{ddd}, J=9.6,2.5,1.4 \mathrm{~Hz}, 1 \mathrm{H}), 7.78(\mathrm{~s}, 1 \mathrm{H}) ;{ }^{13} \mathrm{C} \mathrm{NMR}\left(101 \mathrm{MHz}, \mathrm{DMSO}-d_{6}\right)$ 
$\delta 165.12(\mathrm{~d}, J=2.4 \mathrm{~Hz}), 162.04(\mathrm{~d}, J=247.5 \mathrm{~Hz}), 138.39(\mathrm{~d}, J=7.3 \mathrm{~Hz}), 128.11(\mathrm{~d}, J=3.1 \mathrm{~Hz})$, 122.37 (d, $J=25.7 \mathrm{~Hz}), 120.16(\mathrm{~d}, J=22.9 \mathrm{~Hz}), 117.69$ (d, $J=3.1 \mathrm{~Hz}), 113.52$ (d, $J=9.9 \mathrm{~Hz})$.

3-Fluoro-5-(1,2,4,5-tetrazin-3-yl)benzamide

The compound was obtained from 5-cyano-3-fluorobenzamide $(0.75 \mathrm{~g}, 4.57 \mathrm{mmol})$ following the procedure employed for 2a. Purification by flash chromatography $\left(98 / 2 \mathrm{CH}_{2} \mathrm{Cl}_{2} / \mathrm{MeOH}\right)$ afforded $0.36 \mathrm{~g}(36 \%)$ of $\mathbf{1 3 b}$ as a red solid. $\mathrm{Rf}=0.31$ (Heptane/EtOAc 30/70); ${ }^{1} \mathrm{H}$ NMR (400 MHz, DMSO$\left.d_{6}\right) \delta 10.69(\mathrm{~s}, 1 \mathrm{H}), 8.88(\mathrm{~s}, 1 \mathrm{H}), 8.48-8.20(\mathrm{~m}, 2 \mathrm{H}), 8.16-7.92(\mathrm{~m}, 1 \mathrm{H}), 7.71(\mathrm{~s}, 1 \mathrm{H}) ;{ }^{13} \mathrm{C} \mathrm{NMR}$ $\left(101 \mathrm{MHz}, \mathrm{DMSO}-d_{6}\right) \delta 166.08(\mathrm{~d}, J=2.3 \mathrm{~Hz}), 164.94$ (d, $\left.J=3.3 \mathrm{~Hz}\right), 162.85$ (d, $\left.J=245.6 \mathrm{~Hz}\right)$, 158.89, 138.32 (d, $J=6.9 \mathrm{~Hz}), 134.93(\mathrm{~d}, J=8.2 \mathrm{~Hz}), 123.56$ (d, $J=2.9 \mathrm{~Hz}), 118.85$ (d, $J=23.0$ $\mathrm{Hz}), 117.31(\mathrm{~d}, J=24.1 \mathrm{~Hz}) ; \mathrm{MS}(\mathrm{ESI}) \mathrm{m} / \mathrm{z}[\mathrm{M}+\mathrm{H}]^{+}: 220.09$.

\section{2-Cyano-4-fluorobenzamide (22c)}

To a solution of 2-cyano-4-fluorobenzoic acid $(0.99 \mathrm{~g}, 6.0 \mathrm{mmol})$ in acetonitrile $(20 \mathrm{ml})$ was added $1,1^{\prime}$-carbonyldiimidazole $(1.46 \mathrm{~g}, 9.0 \mathrm{mmol})$. The mixture was stirred at room temperature for $45 \mathrm{~min}$, before addition of aqueous ammonium hydroxide solution $(35 \%, 20 \mathrm{ml})$. The reaction mixture was stirred for $45 \mathrm{~min}$ and ice-cold water $(20 \mathrm{ml})$ was added. The precipitate was collected by filtration and dried to give $0.71 \mathrm{~g}(71 \%)$ of the desired compound as a white solid. $\mathrm{Rf}=0.18$ (Heptane/EtOAc 40/60); ${ }^{1} \mathrm{H}$ NMR (400 MHz, DMSO-d6) $\delta 10.47$ (s, 1H), 9.67 (s, 1H), $8.79-7.87$ (m, 2H), 7.50 (s, $1 \mathrm{H}) ;{ }^{13} \mathrm{C}$ NMR $\left(101 \mathrm{MHz}, \mathrm{DMSO}-d_{6}\right) \delta 172.27(\mathrm{~d}, J=20.8 \mathrm{~Hz}), 165.44(\mathrm{~d}, J=249.8 \mathrm{~Hz}), 163.41(\mathrm{~d}$, $J=9.7 \mathrm{~Hz}), 136.63,129.38,125.29$ (d, $J=9.8 \mathrm{~Hz}), 119.66(\mathrm{~d}, J=23.8 \mathrm{~Hz}), 110.01$ (d, $J=25.5 \mathrm{~Hz})$.

\section{2-Fluoro-N-methyl-4-(1,2,4,5-tetrazin-3-yl)benzamide (14a)}

\section{4-Cyano-2-fluoro-N-methylbenzamide (23a)}

The compound was obtained from 4-cyano-2-fluorobenzoic acid (0.99 g, $6.0 \mathrm{mmol})$ and aqueous methylamine solution $(40 \%, 20 \mathrm{ml})$ as reported above for $22 \mathrm{a}$ to give $0.86 \mathrm{~g}(80 \%)$ of the desired compound as a white solid. $\mathrm{Rf}=0.29\left(\right.$ Heptane/EtOAc 40/60); ${ }^{1} \mathrm{H}$ NMR (400 MHz, DMSO- $\left.d_{6}\right) \delta 8.50$ $(\mathrm{d}, J=6.3 \mathrm{~Hz}, 1 \mathrm{H}), 8.02-7.92(\mathrm{~m}, 1 \mathrm{H}), 7.76(\mathrm{~d}, J=5.9 \mathrm{~Hz}, 2 \mathrm{H}), 2.79(\mathrm{~d}, J=4.6 \mathrm{~Hz}, 3 \mathrm{H}) ;{ }^{13} \mathrm{C} \mathrm{NMR}$ $\left(101 \mathrm{MHz}, \mathrm{DMSO}-d_{6}\right) \delta 163.25,158.82(\mathrm{~d}, J=251.2 \mathrm{~Hz}), 131.56(\mathrm{~d}, J=3.8 \mathrm{~Hz}), 129.54(\mathrm{~d}, J=15.6$ $\mathrm{Hz}), 129.23$ (d, $J=3.9 \mathrm{~Hz}), 120.78(\mathrm{~d}, J=26.6 \mathrm{~Hz}), 117.67(\mathrm{~d}, J=2.9 \mathrm{~Hz}), 114.55(\mathrm{~d}, J=10.1 \mathrm{~Hz})$, 26.69 .

\section{2-Fluoro-N-methyl-4-(1,2,4,5-tetrazin-3-yl)benzamide}

The compound was obtained from 4-cyano-2-fluoro-N-methylbenzamide $(0.77 \mathrm{~g}, 4.32 \mathrm{mmol})$ following the procedure employed for 2a. Purification by flash chromatography (98/2 $\left.\mathrm{CH}_{2} \mathrm{Cl}_{2} / \mathrm{MeOH}\right)$ afforded $0.36 \mathrm{~g}(36 \%)$ of $\mathbf{1 4 a}$ as a red solid. $\mathrm{Rf}=0.36($ Heptane/EtOAc $30 / 70) ;{ }^{1} \mathrm{H}$ 
NMR (400 MHz, DMSO- $\left.d_{6}\right) \delta 10.68(\mathrm{~s}, 1 \mathrm{H}), 8.50(\mathrm{~d}, J=5.3 \mathrm{~Hz}, 1 \mathrm{H}), 8.40(\mathrm{dd}, J=8.1,1.6 \mathrm{~Hz}, 1 \mathrm{H})$, $8.30(\mathrm{dd}, J=11.1,1.6 \mathrm{~Hz}, 1 \mathrm{H}), 7.91(\mathrm{t}, J=7.7 \mathrm{~Hz}, 1 \mathrm{H}), 2.83(\mathrm{~d}, J=4.6 \mathrm{~Hz}, 3 \mathrm{H}) ;{ }^{13} \mathrm{C} \mathrm{NMR}(101$ MHz, DMSO-d $d_{6} \delta 164.83(\mathrm{~d}, J=2.9 \mathrm{~Hz}), 163.79,159.81(\mathrm{~d}, J=249.8 \mathrm{~Hz}), 158.82,136.08(\mathrm{~d}, J=$ $8.5 \mathrm{~Hz}), 131.76$ (d, $J=3.5 \mathrm{~Hz}), 128.25$ (d, $J=15.4 \mathrm{~Hz}), 124.23$ (d, $J=3.3 \mathrm{~Hz}), 115.57$ (d, $J=25.4$ $\mathrm{Hz})$, 26.75; MS (ESI) m/z [M+ H] $]^{+}: 234.09$.

\section{3-Fluoro-N-methyl-5-(1,2,4,5-tetrazin-3-yl)benzamide (14b)}

5-Cyano-3-fluoro-N-methylbenzamide (23b)

The compound was obtained from 5-cyano-3-fluorobenzoic acid (0.99 g, $6.0 \mathrm{mmol})$ and aqueous methylamine solution $(40 \%, 20 \mathrm{ml})$ as reported above for $22 \mathrm{a}$ to $0.81 \mathrm{~g}(76 \%)$ of the desired compound as a white solid. $\mathrm{Rf}=0.32\left(\right.$ Heptane/EtOAc 40/60); ${ }^{1} \mathrm{H}$ NMR (400 MHz, DMSO- $\left.d_{6}\right) \delta$ $8.72(\mathrm{~d}, J=5.8 \mathrm{~Hz}, 1 \mathrm{H}), 8.11(\mathrm{t}, J=1.5 \mathrm{~Hz}, 1 \mathrm{H}), 8.05(\mathrm{ddd}, J=8.1,2.7,1.4 \mathrm{~Hz}, 1 \mathrm{H}), 7.97$ (ddd, $J=$ 9.5, 2.7, $1.5 \mathrm{~Hz}, 1 \mathrm{H}), 2.81(\mathrm{~d}, J=4.6 \mathrm{~Hz}, 3 \mathrm{H}) ;{ }^{13} \mathrm{C}$ NMR (101 MHz, DMSO-d6) $\delta 163.96(\mathrm{~d}, J=2.5$ Hz), 162.02 (d, $J=247.5 \mathrm{~Hz}), 138.49$ (d, $J=7.4 \mathrm{~Hz}), 127.76$ (d, $J=3.3 \mathrm{~Hz}), 122.17$ (d, $J=25.6 \mathrm{~Hz})$, $119.85(\mathrm{~d}, J=23.1 \mathrm{~Hz}), 117.66(\mathrm{~d}, J=3.4 \mathrm{~Hz}), 113.55(\mathrm{~d}, J=10.0 \mathrm{~Hz}), 26.83$.

3-Fluoro-N-methyl-5-(1,2,4,5-tetrazin-3-yl)benzamide

The compound was obtained from 5-cyano-3-fluoro-N-methylbenzamide (0.62 g, $3.48 \mathrm{mmol})$ following the procedure employed for 2a. Purification by flash chromatography (98/2 $\left.\mathrm{CH}_{2} \mathrm{Cl}_{2} / \mathrm{MeOH}\right)$ afforded $0.28 \mathrm{~g}(34 \%)$ of $\mathbf{1 4 b}$ as a red solid. $\mathrm{Rf}=0.31$ (Heptane/EtOAc $\left.30 / 70\right) ;{ }^{1} \mathrm{H}$ NMR (400 MHz, DMSO- $\left.d_{6}\right) \delta 10.70(\mathrm{~s}, 1 \mathrm{H}), 8.87$ (s, 2H), 8.39 (dd, $\left.J=9.3,1.9 \mathrm{~Hz}, 1 \mathrm{H}\right), 7.99(\mathrm{dt}, J$ $=9.5,2.0 \mathrm{~Hz}, 1 \mathrm{H}), 2.85(\mathrm{~d}, J=4.5 \mathrm{~Hz}, 3 \mathrm{H}) ;{ }^{13} \mathrm{C}$ NMR $\left(101 \mathrm{MHz}, \mathrm{DMSO}-d_{6}\right) \delta 164.88(\mathrm{~d}, J=11.1$ $\mathrm{Hz}), 162.89$ (d, $J=245.8 \mathrm{~Hz}), 158.92,138.41$ (d, $J=7.5 \mathrm{~Hz}), 135.00$ (d, $J=8.2 \mathrm{~Hz}), 134.22-131.41$ (m), 123.08, 118.56 (d, $J=22.8 \mathrm{~Hz}), 117.16(\mathrm{~d}, J=24.0 \mathrm{~Hz}), 26.89$; MS (ESI) m/z [M + H] $]^{+}: 234.08$.

\section{2-Cyano-4-fluoro-N-methylbenzamide (23c)}

The compound was obtained from 2-cyano-4-fluorobenzoic acid (0.99 g, $6.0 \mathrm{mmol})$ and aqueous methylamine solution $(40 \%, 20 \mathrm{ml})$ as reported above for $22 \mathrm{a}$ to give $0.74 \mathrm{~g}(69 \%)$ of the desired compound as a white solid. $\mathrm{Rf}=0.24\left(\right.$ Heptane/EtOAc 40/60); ${ }^{1} \mathrm{H}$ NMR (400 MHz, DMSO- $\left.d_{6}\right) \delta$ $10.11(\mathrm{~s}, 0 \mathrm{H}), 9.45(\mathrm{~s}, 0 \mathrm{H}), 8.03(\mathrm{dd}, J=8.4,2.3 \mathrm{~Hz}, 0 \mathrm{H}), 7.82(\mathrm{dd}, J=8.3,4.8 \mathrm{~Hz}, 0 \mathrm{H}), 7.72-7.54$ (m, 0H), $7.72-7.16(\mathrm{~m}, 0 \mathrm{H}), 3.12(\mathrm{~s}, 1 \mathrm{H}) ;{ }^{13} \mathrm{C}$ NMR $\left(101 \mathrm{MHz}, \mathrm{DMSO}-d_{6}\right) \delta 166.97,165.59(\mathrm{~d}, J=$ 250.2 Hz), 158.55, 134.52 (d, $J=10.3 \mathrm{~Hz}), 127.39$ (d, $J=2.5 \mathrm{~Hz}), 125.39$ (d, $J=9.7 \mathrm{~Hz}), 119.64$ (d, $J=23.9 \mathrm{~Hz}), 110.51(\mathrm{~d}, J=25.8 \mathrm{~Hz}), 24.92$. 


\section{2-Fluoro-4-(1,2,4,5-tetrazin-3-yl)aniline (15a)}

\section{Tert-butyl (4-cyano-2-fluorophenyl)carbamate (24a)}

4-Amino-3-fluorobenzonitrile (1.0 g, $7.34 \mathrm{mmol})$ was heated at reflux with $\mathrm{Boc}_{2} \mathrm{O}$ (4.81 g, 22.04 mmol) and DMAP (0.09 g, $0.73 \mathrm{mmol})$ in THF $(50 \mathrm{~mL})$ overnight. The reaction mixture was evaporated to dryness in vacuo, and the residue was dissolved in dichloromethane $(50 \mathrm{~mL})$. TFA (1.6 $\mathrm{mL}$ ) was then added. The mixture was stirred at room temperature for $3 \mathrm{~h}$. The mixture was made basic using concentrated aqueous ammonia, and then extracted with water $(3 \times 30 \mathrm{~mL})$. The organic portion was dried over anhydrous $\mathrm{Na}_{2} \mathrm{SO}_{4}$, and the solvents were evaporated to dryness under reduced pressure. The crude was purified by flash chromatography (90/10 Heptane/EtOAc) to give $1.21 \mathrm{~g}$ $(70 \%)$ of the selected compound as a white solid. $\mathrm{Rf}=0.31(90 / 10 \mathrm{Heptane} / \mathrm{EtOAc}) ;{ }^{1} \mathrm{H}$ NMR $(400$ MHz, DMSO-d $)_{6} \delta 9.54(\mathrm{~s}, 1 \mathrm{H}), 7.99(\mathrm{t}, J=8.3 \mathrm{~Hz}, 1 \mathrm{H}), 7.82(\mathrm{dd}, J=10.9,1.9 \mathrm{~Hz}, 1 \mathrm{H}), 7.67-7.56$ $(\mathrm{m}, 1 \mathrm{H}), 1.48(\mathrm{~s}, 9 \mathrm{H}) ;{ }^{13} \mathrm{C} \mathrm{NMR}\left(101 \mathrm{MHz}, \mathrm{DMSO}-d_{6}\right) \delta 152.87,152.36(\mathrm{~d}, J=247.9 \mathrm{~Hz}), 132.59$ $(\mathrm{d}, J=11.0 \mathrm{~Hz}), 129.70(\mathrm{~d}, J=3.5 \mathrm{~Hz}), 122.96(\mathrm{~d}, J=2.7 \mathrm{~Hz}), 119.82(\mathrm{~d}, J=23.3 \mathrm{~Hz}), 118.43(\mathrm{~d}, J$ $=2.6 \mathrm{~Hz}), 105.64(\mathrm{~d}, J=9.3 \mathrm{~Hz}), 80.91,28.38$.

\section{Tert-butyl (2-fluoro-4-(1,2,4,5-tetrazin-3-yl)phenyl)carbamate (29a)}

The compound was obtained from tert-butyl (4-cyano-2-fluorophenyl)carbamate (1.15 g, $4.87 \mathrm{mmol})$ following the procedure employed for 2a. Purification by flash chromatography (90/10 Heptane/EtOAc) afforded $0.31 \mathrm{~g}(22 \%)$ of the desired compound as a red solid. $\mathrm{Rf}=0.41$ (Heptane/EtOAc 80/20); ${ }^{1} \mathrm{H}$ NMR (600 MHz, Chloroform-d) $\delta 10.19$ (s, 1H), 8.45 (d, $J=1.8 \mathrm{~Hz}$, 2H), $8.35(\mathrm{dd}, J=12.1,1.8 \mathrm{~Hz}, 1 \mathrm{H}), 7.02$ (d, $J=3.9 \mathrm{~Hz}, 1 \mathrm{H}), 1.58(\mathrm{~s}, 9 \mathrm{H}) ;{ }^{13} \mathrm{C} \mathrm{NMR}(151 \mathrm{MHz}$, Chloroform- $d$ ) $\delta 165.44(\mathrm{~d}, J=3.3 \mathrm{~Hz}), 157.57(\mathrm{~d}, J=2.8 \mathrm{~Hz}), 151.84(\mathrm{~d}, J=243.2 \mathrm{~Hz}), 151.80$, $131.86(\mathrm{~d}, J=9.9 \mathrm{~Hz}), 125.74(\mathrm{~d}, J=7.9 \mathrm{~Hz}), 125.20(\mathrm{~d}, J=2.9 \mathrm{~Hz}), 119.67,114.38(\mathrm{~d}, J=22.0$ $\mathrm{Hz}), 81.96,28.23$.

\section{2-Fluoro-4-(1,2,4,5-tetrazin-3-yl)aniline}

To a solution of tert-butyl (2-fluoro-4-(1,2,4,5-tetrazin-3-yl)phenyl)carbamate $(0.20 \mathrm{~g}, 0.69 \mathrm{mmol})$ in $\mathrm{CH}_{2} \mathrm{Cl}_{2}(4 \mathrm{~mL})$ was added TFA $(4 \mathrm{~mL})$. The reaction was stirred at room temperature for $1 \mathrm{~h}$. The solvent was then evaporated under reduced pressure to give $0.14 \mathrm{~g}$ of crude. Purification by flash chromatography (70/30 Heptane/EtOAc) afforded $0.12 \mathrm{~g} \mathrm{(91 \% )} \mathrm{of} \mathbf{1 5 a}$ as a red solid. $\mathrm{Rf}=0.33(70 / 30$ Heptane/EtOAc); ${ }^{1} \mathrm{H}$ NMR (400 MHz, Chloroform- $d$ ) $\delta 10.10$ (s, 1H), $8.78-8.05$ (m, 2H), 6.94 (t, $J$ $=8.6 \mathrm{~Hz}, 1 \mathrm{H}), 4.17(\mathrm{~s}, 2 \mathrm{H}) ;{ }^{13} \mathrm{C} \mathrm{NMR}(101 \mathrm{MHz}$, Chloroform- $d) \delta 165.77(\mathrm{~d}, J=3.1 \mathrm{~Hz}), 157.12$, $151.28(\mathrm{~d}, J=239.9 \mathrm{~Hz}), 139.71(\mathrm{~d}, J=12.9 \mathrm{~Hz}), 125.55(\mathrm{~d}, J=2.9 \mathrm{~Hz}), 121.33(\mathrm{~d}, J=7.3 \mathrm{~Hz})$, $116.39(\mathrm{~d}, J=4.0 \mathrm{~Hz}), 115.12$ (d, $J=20.9 \mathrm{~Hz})$; MS (ESI) m/z [M + H] $]^{+} 192.09$. 


\section{3-Fluoro-5-(1,2,4,5-tetrazin-3-yl)aniline (15b)}

\section{Tert-butyl (5-cyano-3-fluorophenyl)carbamate (24b)}

The compound was obtained from 3-amino-5-fluorobenzonitrile $(1.0 \mathrm{~g}, 7.34 \mathrm{mmol})$ as described above for 24a. The crude was purified by flash chromatography (90/10 Heptane/EtOAc) to give 1.23 $\mathrm{g}(71 \%)$ of the desired compound as a white solid. $\mathrm{Rf}=0.32(90 / 10 \mathrm{Heptane} / \mathrm{EtOAc}) ;{ }^{1} \mathrm{H}$ NMR (400 $\mathrm{MHz}$, Chloroform- $d$ ) $\delta 7.37-7.29(\mathrm{~m}, 2 \mathrm{H}), 7.19(\mathrm{dt}, J=8.9,2.2 \mathrm{~Hz}, 1 \mathrm{H}), 1.46(\mathrm{~s}, 9 \mathrm{H}) ;{ }^{13} \mathrm{C}$ NMR $(101 \mathrm{MHz}$, Chloroform- $d$ ) $\delta 162.00(\mathrm{~d}, J=251.2 \mathrm{~Hz}), 150.67,141.84(\mathrm{~d}, J=10.5 \mathrm{~Hz}), 128.07(\mathrm{~d}, J$ $=3.6 \mathrm{~Hz}), 120.90(\mathrm{~d}, J=22.6 \mathrm{~Hz}), 118.12(\mathrm{~d}, J=24.6 \mathrm{~Hz}), 116.85(\mathrm{~d}, J=3.5 \mathrm{~Hz}), 113.74(\mathrm{~d}, J=$ $10.8 \mathrm{~Hz}), 84.12,27.86$.

Tert-butyl (3-fluoro-5-(1,2,4,5-tetrazin-3-yl)phenyl) carbamate (29b)

The compound was obtained from tert-butyl (5-cyano-3-fluorophenyl)carbamate (0.94 g, $4.00 \mathrm{mmol})$ following the procedure employed for 2a. Purification by flash chromatography (90/10 Heptane/EtOAc) afforded $0.33 \mathrm{~g}(28 \%)$ of the desired compound as a red solid. $\mathrm{Rf}=0.36$ (Heptane/EtOAc 80/20); ${ }^{1} \mathrm{H}$ NMR (400 MHz, DMSO- $\left.d_{6}\right) \delta 10.63(\mathrm{~s}, 1 \mathrm{H}), 9.95(\mathrm{~s}, 1 \mathrm{H}), 8.54(\mathrm{t}, J=$ $1.7 \mathrm{~Hz}, 1 \mathrm{H}), 7.82(\mathrm{ddd}, J=9.4,2.5,1.4 \mathrm{~Hz}, 1 \mathrm{H}), 7.64(\mathrm{dt}, J=11.2,2.3 \mathrm{~Hz}, 1 \mathrm{H}), 1.52(\mathrm{~s}, 9 \mathrm{H}) ;{ }^{13} \mathrm{C}$ NMR (101 MHz, DMSO-d $)) \delta 165.12(\mathrm{~d}, J=3.8 \mathrm{~Hz}), 163.18$ (d, $J=241.7 \mathrm{~Hz}), 158.81,153.08$, $142.96(\mathrm{~d}, J=11.6 \mathrm{~Hz}), 134.64(\mathrm{~d}, J=10.1 \mathrm{~Hz}), 113.57$ (d, $J=2.6 \mathrm{~Hz}), 108.96$ (d, $J=26.6 \mathrm{~Hz})$, $107.88(\mathrm{~d}, J=24.3 \mathrm{~Hz}), 80.49,28.50$.

\section{3-Fluoro-5-(1,2,4,5-tetrazin-3-yl)aniline}

To a solution of tert-butyl (3-fluoro-5-(1,2,4,5-tetrazin-3-yl)phenyl)carbamate ( $0.15 \mathrm{~g}, 0.51 \mathrm{mmol})$ in $\mathrm{CH}_{2} \mathrm{Cl}_{2}(4 \mathrm{~mL})$ was added TFA $(4 \mathrm{~mL})$. The reaction was stirred at room temperature for $1 \mathrm{~h}$. The solvent was then evaporated under reduced pressure to give $0.11 \mathrm{~g}$ of crude. Purification by flash chromatography (70/30 Heptane/EtOAc) afforded $0.45 \mathrm{~g}(46 \%)$ of $\mathbf{1 5 b}$ as a red solid. $\mathrm{Rf}=0.31(70 / 30$ Heptane/EtOAc); ${ }^{1} \mathrm{H}$ NMR (400 MHz, Methanol- $\left.d_{4}\right) \delta 10.31(\mathrm{~s}, 1 \mathrm{H}), 7.72(\mathrm{t}, J=1.7 \mathrm{~Hz}, 1 \mathrm{H}), 7.48$ $(\mathrm{dt}, J=9.6,1.9 \mathrm{~Hz}, 1 \mathrm{H}), 6.66(\mathrm{dt}, J=10.9,2.3 \mathrm{~Hz}, 1 \mathrm{H}) ;{ }^{13} \mathrm{C} \mathrm{NMR}\left(101 \mathrm{MHz}\right.$, Methanol- $\left.d_{4}\right) \delta 165.87$ (d, $J=3.8 \mathrm{~Hz}), 164.41$ (d, $J=241.6 \mathrm{~Hz}), 157.91,151.23$ (d, $J=11.5 \mathrm{~Hz}), 134.13$ (d, $J=10.8 \mathrm{~Hz})$, $109.48(\mathrm{~d}, J=2.0 \mathrm{~Hz}), 104.62(\mathrm{~d}, J=25.0 \mathrm{~Hz}), 102.28(\mathrm{~d}, J=25.2 \mathrm{~Hz}) ; \mathrm{MS}(\mathrm{ESI}) \mathrm{m} / \mathrm{z}[\mathrm{M}+\mathrm{H}]^{+}$: 192.11.

\section{Tert-butyl (2-cyano-4-fluorophenyl)carbamate (24c)}

The compound was obtained from 2-amino-5-fluorobenzonitrile (1.0 g, $7.34 \mathrm{mmol})$ as described above for 24a. The crude was purified by flash chromatography (90/10 Heptane/EtOAc) to give 0.81 $\mathrm{g}(47 \%)$ of the desired compound as a white solid. $\mathrm{Rf}=0.41\left(90 / 10\right.$ Heptane/EtOAc); ${ }^{1} \mathrm{H}$ NMR (400 MHz, Chloroform- $d$ ) $\delta 8.12(\mathrm{dd}, J=9.4,4.8 \mathrm{~Hz}, 1 \mathrm{H}), 7.26-7.11(\mathrm{~m}, 2 \mathrm{H}), 6.90(\mathrm{~s}, 1 \mathrm{H}), 1.46(\mathrm{~s}, 9 \mathrm{H})$; 
${ }^{13} \mathrm{C}$ NMR (101 MHz, Chloroform-d) $\delta 157.15(\mathrm{~d}, J=245.5 \mathrm{~Hz}), 151.94,137.95(\mathrm{~d}, J=2.9 \mathrm{~Hz})$, $121.67(\mathrm{~d}, J=22.1 \mathrm{~Hz}), 121.54(\mathrm{~d}, J=7.8 \mathrm{~Hz}), 118.26(\mathrm{~d}, J=25.5 \mathrm{~Hz}), 115.37$ (d, $J=2.7 \mathrm{~Hz}), 101.79$ $(\mathrm{d}, J=9.1 \mathrm{~Hz}), 82.09,28.16$.

\section{N-(2-fluoro-4-(1,2,4,5-tetrazin-3-yl)phenyl)acetamide (16a)}

$\mathrm{N}$-(4-Cyano-2-fluorophenyl)acetamide (25a)

To a solution of 4-amino-3-fluorobenzonitrile $(0.82 \mathrm{~g}, 6.00 \mathrm{mmol})$ in $\mathrm{CH}_{2} \mathrm{Cl}_{2}(30.0 \mathrm{~mL})$ was added acetic anhydride $(0.80 \mathrm{~mL}, 8.40 \mathrm{mmol})$. The mixture was stirred at room temperature for $12 \mathrm{~h}$. The suspension was filtered, and the solvent removed under reduced presuure. Purification by flash chromatography (70/30 Heptane/EtOAc) afforded $0.90 \mathrm{~g}(85 \%)$ of the desired compound as a white solid. Rf $=0.5\left(\right.$ Heptane/EtOAc 60/40); ${ }^{1} \mathrm{H}$ NMR (400 MHz, DMSO- $\left.d_{6}\right) \delta 10.12(\mathrm{~s}, 1 \mathrm{H}), 8.28(\mathrm{t}, J=$ $8.2 \mathrm{~Hz}, 1 \mathrm{H}), 7.88(\mathrm{dd}, J=11.1,1.9 \mathrm{~Hz}, 1 \mathrm{H}), 7.65(\mathrm{dt}, J=8.5,1.3 \mathrm{~Hz}, 1 \mathrm{H}), 2.15(\mathrm{~s}, 3 \mathrm{H}) ;{ }^{13} \mathrm{C} \mathrm{NMR}$ $\left(101 \mathrm{MHz}, \mathrm{DMSO}-d_{6}\right) \delta 169.94,152.05(\mathrm{~d}, J=247.2 \mathrm{~Hz}), 132.10(\mathrm{~d}, J=11.2 \mathrm{~Hz}), 129.82(\mathrm{~d}, J=3.6$ Hz), 123.31 (d, $J=2.9 \mathrm{~Hz}), 119.77$ (d, $J=23.4 \mathrm{~Hz}), 118.36$ (d, $J=2.7 \mathrm{~Hz}), 106.18$ (d, $J=9.4 \mathrm{~Hz})$, 24.31 .

\section{N-(2-Fluoro-4-(1,2,4,5-tetrazin-3-yl)phenyl)acetamide}

The compound was obtained from tert-butyl N-(4-cyano-2-fluorophenyl)acetamide (0.71 g, 4.00 mmol) following the procedure employed for 2a. Purification by flash chromatography (60/40 Heptane/EtOAc) afforded $0.37 \mathrm{~g}(40 \%)$ of $\mathbf{1 6 a}$ as a red solid. $\mathrm{Rf}=0.25($ Heptane/EtOAc $60 / 40) ;{ }^{1} \mathrm{H}$ NMR (400 MHz, DMSO- $\left.d_{6}\right) \delta 10.58(\mathrm{~s}, 1 \mathrm{H}), 10.09(\mathrm{~s}, 1 \mathrm{H}), 8.45-8.21(\mathrm{~m}, 3 \mathrm{H}), 2.18(\mathrm{~s}, 3 \mathrm{H}) ;{ }^{13} \mathrm{C}$ NMR (101 MHz, DMSO-d $d_{6} \delta 169.79,164.91(\mathrm{~d}, J=3.0 \mathrm{~Hz}), 158.44,153.21(\mathrm{~d}, J=246.0 \mathrm{~Hz})$, $131.38(\mathrm{~d}, J=11.3 \mathrm{~Hz}), 128.01(\mathrm{~d}, J=7.9 \mathrm{~Hz}), 124.72$ (d, $J=3.3 \mathrm{~Hz}), 123.67,114.75(\mathrm{~d}, J=22.1$ $\mathrm{Hz}), 24.32 ; \mathrm{MS}(\mathrm{ESI}) \mathrm{m} / \mathrm{z}[\mathrm{M}+\mathrm{H}]^{+}: 234.09$.

\section{N-(3-Fluoro-5-(1,2,4,5-tetrazin-3-yl)phenyl)acetamide (16b)}

$N$-(3-Cyano-5-fluorophenyl)acetamide (25b)

The compound was obtained from 3-amino-5-fluorobenzonitrile $(0.82 \mathrm{~g}, 6.00 \mathrm{mmol})$ as described above for 25a. The crude was purified by flash chromatography (70/30 Heptane/EtOAc) to give 0.92 $\mathrm{g}(86 \%)$ of the desired compound as a white solid. $\mathrm{Rf}=0.31$ (Heptane/EtOAc 60/40); ${ }^{1} \mathrm{H}$ NMR (400 $\left.\mathrm{MHz}, \mathrm{DMSO}-d_{6}\right) \delta 10.45(\mathrm{~s}, 1 \mathrm{H}), 7.86-7.70(\mathrm{~m}, 2 \mathrm{H}), 7.57-7.37(\mathrm{~m}, 1 \mathrm{H}), 2.09(\mathrm{~s}, 3 \mathrm{H}) ;{ }^{13} \mathrm{C} \mathrm{NMR}$ $\left(101 \mathrm{MHz}, \mathrm{DMSO}-d_{6}\right) \delta 169.69,162.24(\mathrm{~d}, J=244.3 \mathrm{~Hz}), 142.35$ (d, $\left.J=11.8 \mathrm{~Hz}\right), 118.65,118.09$ (d, $J=3.6 \mathrm{~Hz}), 113.70(\mathrm{~d}, J=25.5 \mathrm{~Hz}), 113.25(\mathrm{~d}, J=12.1 \mathrm{~Hz}), 110.95(\mathrm{~d}, J=26.2 \mathrm{~Hz}), 24.52$.

$\mathrm{N}$-(3-Fluoro-5-(1,2,4,5-tetrazin-3-yl)phenyl)acetamide 
The compound was obtained from tert-butyl N-(3-cyano-5-fluorophenyl)acetamide (0.58 g, 3.25 mmol) following the procedure employed for 2a. Purification by flash chromatography (60/40 Heptane/EtOAc) afforded $0.19 \mathrm{~g}(25 \%)$ of $\mathbf{1 6 b}$ as a red solid. $\mathrm{Rf}=0.24($ Heptane/EtOAc $60 / 40) ;{ }^{1} \mathrm{H}$ NMR (400 MHz, DMSO- $\left.d_{6}\right) \delta 10.64(\mathrm{~s}, 1 \mathrm{H}), 10.48(\mathrm{~s}, 1 \mathrm{H}), 8.52(\mathrm{t}, J=1.7 \mathrm{~Hz}, 1 \mathrm{H}), 7.98-7.81(\mathrm{~m}$, 2H), $2.12(\mathrm{~s}, 3 \mathrm{H}) ;{ }^{13} \mathrm{C}$ NMR $\left(101 \mathrm{MHz}, \mathrm{DMSO}-d_{6}\right) \delta 169.54,165.03(\mathrm{~d}, J=3.8 \mathrm{~Hz}), 163.08(\mathrm{~d}, J=$ $242.2 \mathrm{~Hz}), 158.83,142.45(\mathrm{~d}, J=11.5 \mathrm{~Hz}), 134.61$ (d, $J=10.1 \mathrm{~Hz}), 114.40$ (d, $J=2.6 \mathrm{~Hz}), 109.87$ (d, $J=26.6 \mathrm{~Hz}), 108.74(\mathrm{~d}, J=24.4 \mathrm{~Hz}), 24.58 ; \mathrm{MS}(\mathrm{ESI}) \mathrm{m} / \mathrm{z}[\mathrm{M}+\mathrm{H}]^{+}: 234.08$.

\section{N-(2-cyano-4-fluorophenyl)acetamide (25c)}

The compound was obtained from 2-amino-5-fluorobenzonitrile (0.82 g, $6.00 \mathrm{mmol})$ as described above for N-(4-cyano-2-fluorophenyl)acetamide. The crude was purified by flash chromatography (70/30 Heptane/EtOAc) to give $0.81 \mathrm{~g}(76 \%)$ of the desired compound as a white solid. $\mathrm{Rf}=0.41$ (Heptane/EtOAc 60/40); ${ }^{1} \mathrm{H}$ NMR (400 MHz, DMSO-d $\left.)\right) \delta 10.16(\mathrm{~s}, 1 \mathrm{H}), 7.81$ (dd, $J=8.4,1.8 \mathrm{~Hz}$, 1H), $7.58(\mathrm{dd}, J=6.7,1.7 \mathrm{~Hz}, 2 \mathrm{H}), 2.09$ (s, 3H); ${ }^{13} \mathrm{C}$ NMR (101 MHz, DMSO-d 6 ) $\delta 169.22,158.86$ $(\mathrm{d}, J=244.3 \mathrm{~Hz}), 137.55(\mathrm{~d}, J=3.0 \mathrm{~Hz}), 128.41(\mathrm{~d}, J=8.7 \mathrm{~Hz}), 121.74$ (d, $J=22.4 \mathrm{~Hz}), 120.01$ (d, $J=26.0 \mathrm{~Hz}), 116.21(\mathrm{~d}, J=2.7 \mathrm{~Hz}), 109.34,23.49$.

\section{2-Fluoro-4-(1,2,4,5-tetrazin-3-yl)benzenesulfonamide (17a)}

\section{4-Cyano-2-fluorobenzene-1-sulfonyl chloride (26a)}

The compound was synthesized as describe in the literature. ${ }^{[14]}(30)$. A portion of glacial acetic acid $(30 \mathrm{~mL})$ was saturated for $30 \mathrm{~min}$ with gaseous sulfur dioxide. To the resulting solution, cooled on an ice bath, was added under stirring an aqueous solution of $\mathrm{CuCl}_{2}(1.5 \mathrm{~g}$ in $10 \mathrm{~mL}$ ) (suspension $\mathrm{A}$ ). 4-amino-3-fluorobenzonitrile (5 g, $36.75 \mathrm{mmol}$ ) was dissolved in a mixture of glacial acetic acid (30 $\mathrm{mL})$ and concentrated $\mathrm{HCl}(15 \mathrm{~mL})$. To the resulting solution, cooled in an ice-salt bath $\left(-5^{\circ} \mathrm{C}\right)$, was added dropwise under stirring an aqueous solution of $\mathrm{NaNO}_{2}(3.37 \mathrm{~g}$ in $10 \mathrm{~mL}, 48.88 \mathrm{mmol})$. At the end of the addition, the resulting solution was slowly mixed with suspension A. After being stirred for $15 \mathrm{~min}$, the suspension was poured onto ice. The resulting precipitate was collected by filtration, washed with water to give $3.09 \mathrm{~g}(40 \%)$ of the desired compound as a white solid. ${ }^{1} \mathrm{H}$ NMR (400 $\mathrm{MHz}$, Chloroform- $d$ ) $\delta 8.15(\mathrm{dd}, J=8.2,6.8 \mathrm{~Hz}, 1 \mathrm{H}), 7.90-7.22(\mathrm{~m}, 1 \mathrm{H}) ;{ }^{13} \mathrm{C} \mathrm{NMR}(101 \mathrm{MHz}$, Chloroform- $d$ ) $\delta 158.25(\mathrm{~d}, J=266.7 \mathrm{~Hz}), 135.53(\mathrm{~d}, J=13.0 \mathrm{~Hz}), 130.36,128.61(\mathrm{~d}, J=4.7 \mathrm{~Hz})$, $121.95(\mathrm{~d}, J=23.9 \mathrm{~Hz}), 120.92(\mathrm{~d}, J=9.4 \mathrm{~Hz}), 115.43$ (d, $J=2.6 \mathrm{~Hz})$.

\section{4-Cyano-2-fluorobenzenesulfonamide (27a)}

To a solution of 4-cyano-2-fluorobenzene-1-sulfonyl chloride $(0.60 \mathrm{~g}, 2.73 \mathrm{mmol})$ in $\mathrm{CH}_{3} \mathrm{CN}$ at $0{ }^{\circ} \mathrm{C}$ was added dropwise a solution of $\mathrm{NH}_{3}$ in $\mathrm{MeOH}(5.00 \mathrm{~mL}, 7 \mathrm{M})$. The reaction was stirred at Rt for 2 
hours and then the solvent was removed under reduced pressure. The residue was solubilized in EtOAc $(30 \mathrm{~mL})$ and washed with water $(2 \times 30 \mathrm{~mL})$. The organic phase was dried over $\mathrm{Na}_{2} \mathrm{SO}_{3}$, filtered and concentrated under reduced pressure to give $0.51 \mathrm{~g}(93 \%)$ of the desired compound as a white solid. Rf: 0.38 (Heptane/EtOAc 40/60); ${ }^{1} \mathrm{H}$ NMR (400 MHz, DMSO- $\left.d_{6}\right) \delta 8.12$ $(\mathrm{dd}, J=10.0,1.5 \mathrm{~Hz}, 1 \mathrm{H}), 8.03-7.92(\mathrm{~m}, 3 \mathrm{H}), 7.89(\mathrm{dd}, J=8.1,1.5 \mathrm{~Hz}, 1 \mathrm{H}) ;{ }^{13} \mathrm{C} \mathrm{NMR}(101 \mathrm{MHz}$, DMSO- $\left.d_{6}\right) \delta 158.01(\mathrm{~d}, J=255.5 \mathrm{~Hz}), 136.37(\mathrm{~d}, J=14.7 \mathrm{~Hz}), 129.94,129.64(\mathrm{~d}, J=4.3 \mathrm{~Hz}), 121.76$ (d, $J=25.5 \mathrm{~Hz}), 117.21(\mathrm{~d}, J=2.6 \mathrm{~Hz}), 116.77(\mathrm{~d}, J=9.8 \mathrm{~Hz})$.

\section{2-Fluoro-4-(1,2,4,5-tetrazin-3-yl)benzenesulfonamide}

The compound was obtained from 4-cyano-2-fluorobenzenesulfonamide $(0.51 \mathrm{~g}, 2.54 \mathrm{mmol})$ following the procedure employed for 2a. Purification by flash chromatography (98/2 $\left.\mathrm{CH}_{2} \mathrm{Cl} / \mathrm{MeOH}\right)$ afforded $0.26 \mathrm{~g}(40 \%)$ of $\mathbf{1 7 a}$ as a red solid. $\mathrm{Rf}=0.43($ Heptane/EtOAc $40 / 60) ;{ }^{1} \mathrm{H}$ NMR (400 MHz, DMSO-d $\left.d_{6}\right) 10.71(\mathrm{~s}, 1 \mathrm{H}), 8.50(\mathrm{dd}, J=8.2,1.6 \mathrm{~Hz}, 1 \mathrm{H}), 8.43(\mathrm{dd}, J=10.9,1.6$

$\mathrm{Hz}, 1 \mathrm{H}), 8.11(\mathrm{t}, J=7.8 \mathrm{~Hz}, 1 \mathrm{H}), 7.92(\mathrm{~s}, 2 \mathrm{H}) ;{ }^{13} \mathrm{C}$ NMR $\left(101 \mathrm{MHz}, \mathrm{DMSO}-d_{6}\right) \delta 164.56,158.89$, $158.86(\mathrm{~d}, J=254.2 \mathrm{~Hz}), 138.15(\mathrm{~d}, J=8.1 \mathrm{~Hz}), 135.35(\mathrm{~d}, J=14.9 \mathrm{~Hz}), 130.14,124.44$ (d, $J=4.0$ $\mathrm{Hz}), 116.42(\mathrm{~d}, J=24.2 \mathrm{~Hz})$; MS (ESI) $\mathrm{m} / \mathrm{z}[\mathrm{M}+\mathrm{H}]^{+}: 256.05$.

\section{3-Fluoro-5-(1,2,4,5-tetrazin-3-yl)benzenesulfonamide (17b)}

\section{3-Cyano-5-fluorobenzene-1-sulfonyl chloride (26b)}

The compound was obtained from 3-amino-5-fluorobenzonitrile $(5 \mathrm{~g}, 36.75 \mathrm{mmol})$ as described above for 26a to give 2.98 (38\%) of the desired compound as a white solid. ${ }^{1} \mathrm{H}$ NMR (400 MHz, Chloroform- $d$ ) $\delta 8.17(\mathrm{~s}, 1 \mathrm{H}), 8.02(\mathrm{dt}, J=7.0,2.1 \mathrm{~Hz}, 1 \mathrm{H}), 7.77(\mathrm{ddd}, J=7.4,2.5,1.3 \mathrm{~Hz}, 2 \mathrm{H}) ;{ }^{13} \mathrm{C}$ NMR (101 MHz, Chloroform- $d) \delta 162.01(\mathrm{~d}, J=259.6 \mathrm{~Hz}), 146.83(\mathrm{~d}, J=7.4 \mathrm{~Hz}), 126.44(\mathrm{~d}, J=$ $4.2 \mathrm{~Hz}), 125.68(\mathrm{~d}, J=24.4 \mathrm{~Hz}), 119.00(\mathrm{~d}, J=25.1 \mathrm{~Hz}), 116.14(\mathrm{~d}, J=8.9 \mathrm{~Hz}), 115.13(\mathrm{~d}, J=2.7$ $\mathrm{Hz})$.

\section{3-Cyano-5-fluorobenzenesulfonamide (27b)}

The compound was obtained from 3-cyano-5-fluorobenzene-1-sulfonyl chloride ( $0.60 \mathrm{~g}, 2.73 \mathrm{mmol})$ as described above for $27 \mathrm{a}$ to give $0.50 \mathrm{~g}(91 \%)$ of the desired compound as a white solid. $\mathrm{Rf}=0.31$ (Heptane/EtOAc 40/60); ${ }^{1} \mathrm{H}$ NMR (400 MHz, DMSO-d6) $\delta 8.17$ (dt, $\left.J=8.5,1.9 \mathrm{~Hz}, 1 \mathrm{H}\right), 8.11(\mathrm{~d}, J$ $=1.4 \mathrm{~Hz}, 1 \mathrm{H}), 8.01(\mathrm{dt}, J=8.2,2.0 \mathrm{~Hz}, 1 \mathrm{H}), 7.85(\mathrm{~s}, 2 \mathrm{H}),{ }^{13} \mathrm{C}$ NMR $\left(101 \mathrm{MHz}, \mathrm{DMSO}-d_{6}\right) \delta 161.79$ $(\mathrm{d}, J=251.2 \mathrm{~Hz}), 147.98(\mathrm{~d}, J=7.0 \mathrm{~Hz}), 126.25$ (d, $J=3.5 \mathrm{~Hz}), 123.40$ (d, $J=25.6 \mathrm{~Hz}), 118.48$ (d, $J=24.2 \mathrm{~Hz}), 117.03$ (d, $J=3.0 \mathrm{~Hz}), 114.31(\mathrm{~d}, J=10.0 \mathrm{~Hz})$.

3-Fluoro-5-(1,2,4,5-tetrazin-3-yl)benzenesulfonamide

The compound was obtained from 3-cyano-5-fluorobenzenesulfonamide (0.49 g, $2.45 \mathrm{mmol})$ following the procedure employed for 2a. Purification by flash chromatography (98/2 
$\left.\mathrm{CH}_{2} \mathrm{Cl} 2 / \mathrm{MeOH}\right)$ afforded $0.21 \mathrm{~g}(37 \%)$ of $\mathbf{1 7 b}$ as a red solid. $\mathrm{Rf}=0.25($ Heptane/EtOAc $60 / 40) ;{ }^{1} \mathrm{H}$ NMR (400 MHz, DMSO- $\left.d_{6}\right) \delta 10.73(\mathrm{~s}, 1 \mathrm{H}), 8.81(\mathrm{t}, J=1.5 \mathrm{~Hz}, 1 \mathrm{H}), 8.49$ (ddd, $J=9.3,2.5,1.4 \mathrm{~Hz}$, 1H), 7.95 (ddd, $J=8.1,2.5,1.6 \mathrm{~Hz}, 1 \mathrm{H}), 7.76(\mathrm{~s}, 2 \mathrm{H}) ;{ }^{13} \mathrm{C} \mathrm{NMR}\left(101 \mathrm{MHz}, \mathrm{DMSO}-d_{6}\right) \delta 164.47$ (d, $J=2.9 \mathrm{~Hz}), 162.61$ (d, $J=249.5 \mathrm{~Hz}), 159.00,147.99$ (d, $J=6.8 \mathrm{~Hz}), 135.87$ (d, $J=8.2 \mathrm{~Hz}), 121.44$ (d, $J=3.0 \mathrm{~Hz}), 118.06(\mathrm{~d}, J=24.2 \mathrm{~Hz}), 117.10(\mathrm{~d}, J=24.4 \mathrm{~Hz})$; MS (ESI) m/z [M + H] $]^{+}: 256.07$.

\section{2-Cyano-4-fluorobenzenesulfonamide}

\section{2-Cyano-4-fluorobenzene-1-sulfonyl chloride (26c)}

The compound was obtained from 2-amino-5-fluorobenzonitrile (5 g, $36.75 \mathrm{mmol})$ as described above for 26a to give 2.41 (30\%) of the desired compound as a white solid. ${ }^{1} \mathrm{H}$ NMR (400 MHz, Chloroform- $d$ ) $\delta 8.15(\mathrm{dd}, J=8.2,6.8 \mathrm{~Hz}, 1 \mathrm{H}), 7.78-7.69(\mathrm{~m}, 2 \mathrm{H}) ;{ }^{13} \mathrm{C}$ NMR (101 MHz, Chloroformd) $\delta 158.25(\mathrm{~d}, J=266.7 \mathrm{~Hz}), 135.53(\mathrm{~d}, J=13.0 \mathrm{~Hz}), 130.36,128.61(\mathrm{~d}, J=4.7 \mathrm{~Hz}), 121.95(\mathrm{~d}, J=$ $23.9 \mathrm{~Hz}), 120.92(\mathrm{~d}, J=9.4 \mathrm{~Hz}), 115.43(\mathrm{~d}, J=2.6 \mathrm{~Hz})$.

\section{2-Cyano-4-fluorobenzenesulfonamide (27c)}

The compound was obtained from 2-cyano-4-fluorobenzene-1-sulfonyl chloride ( $0.60 \mathrm{~g}, 2.73 \mathrm{mmol})$ as described above for $27 \mathrm{a}$ to give $0.48 \mathrm{~g}(88 \%)$ of the desired compound as a white solid. $\mathrm{Rf}=0.35$ (Heptane/EtOAc 40/60); ${ }^{1} \mathrm{H}$ NMR (400 MHz, DMSO-d6) $\delta 9.04$ (s, 1H), 8.90 (s, 1H), 8.15 - 7.96 (m, 2H), $7.69(\mathrm{td}, J=8.6,2.3 \mathrm{~Hz}, 1 \mathrm{H}) ;{ }^{13} \mathrm{C} \mathrm{NMR}\left(101 \mathrm{MHz}, \mathrm{DMSO}-d_{6}\right) \delta 164.96(\mathrm{~d}, J=250.9 \mathrm{~Hz})$, 159.95, 138.90, 131.53 (d, $J=9.7 \mathrm{~Hz}), 123.95$ (d, $J=9.9 \mathrm{~Hz}), 121.13$ (d, $J=24.1 \mathrm{~Hz}), 111.22(\mathrm{~d}, J$ $=26.0 \mathrm{~Hz})$.

\section{Stopped-flow Kinetic measurements}

Stopped-flow measurements were performed using an SX20-LED stopped-flow spectrophotometer (Applied Photophysics) equipped with a 535nm LED (optical pathlength $10 \mathrm{~mm}$, full width halfmaximum $34 \mathrm{~nm})$ to monitor the characteristic tetrazine visible light absorbance $(520-540 \mathrm{~nm})$. Solutions of TCO in anhydrous $\mathrm{CH}_{3} \mathrm{CN}$ and axTCO-PEG 4 in DPBS were prepared at an approximate concentration above $2 \mathrm{mM} \cdot{ }^{[17]}$ The exact concentration was determined by absorbance titration with 3,6-dimethyltetrazine. ${ }^{[22]}$ These initial stock solutions were diluted before stopped-flow analysis to reach a final TCO concentration of $2 \mathrm{mM}$. Stock solutions of tetrazines were prepared in DMSO at a concentration of $10 \mathrm{mM}$. Serial dilution into $\mathrm{CH}_{3} \mathrm{CN}$ or DPBS was used to prepare solutions for stopped-flow analysis at a Tz concentration of $100 \mu \mathrm{M}$. The reagent syringes were loaded with solutions of the Tz and TCO or axTCO-PEG 4 and the instrument was primed. Subsequent data were collected in triplicate to sextuplicate for each tetrazine. Reactions were conducted at $25^{\circ} \mathrm{C}\left(\mathrm{CH}_{3} \mathrm{CN}\right)$ or $37^{\circ} \mathrm{C}$ (DPBS) and recorded automatically at the time of acquisition. Data sets were analyzed by 
fitting an exponential decay using Prism 6 (Graphpad) to calculate the observed pseudo-first order rate constants that were converted into second order rate constants by dividing through the concentration of excess TCO compound.

\section{DFT calculations}

Density functional theory calculations were performed in Gaussian 16 Revision A.03. The $\omega$ B97XD functional was used in combination with the def2-TZVPD basis set. ${ }^{[23]}$ The basis set definition was obtained from the basis set exchange. ${ }^{[24]}$ Solvent effects were included using the SMD model. Conformer searches of all stationary points were conducted using CREST and all obtained conformers were reoptimized using $\omega$ B97X-D/def2-TZVPD with or without solvation. ${ }^{[25]}$ Stationary points were confirmed by having zero (minima) or exactly one (transition states) imaginary frequency. Free energies were corrected using the Truhlar quasiharmonic approximation with a cutoff of $100 \mathrm{~cm}^{-1}$ in GoodVibes. ${ }^{[26]}$

pKa calculations were conducted using the method introduced by Shields and coworkers. ${ }^{[19]}$ Structures were optimized using $\mathrm{HF} / 6-31+\mathrm{G}(\mathrm{d})$ with the CPCM solvent models and single point energies were calculated at the HF/6-31G(d) level of theory, with or without CPCM solvation to determine the solvation energy. CBS-QB3 was used to obtain accurate free energies and corrected using the HF calculated solvation energies. Equation 8 in the manuscript by Shields and coworkers was used to estimate the absolute $\mathrm{pKa}$ in water. ${ }^{[19]}$

\section{Supporting Information}

The Supporting Information is available free of charge on the publisher website at DOI:

\section{Acknowledgments}

This project has received funding from the European Union's EU Framework Programme for Research and Innovation Horizon 2020, under grant agreement no. 668532 and from the European Union's Horizon 2020 research and innovation program under the Marie Skłodowska-Curie grant agreement No. 813528. HM and MMH have received funding from the European Union's EU Framework Programme for Research and Innovation Horizon 2020 (grant agreement no. 670261). The Lundbeck Foundation, the Novo Nordisk Foundation, the Innovation Fund Denmark, and the Research Council for Independent Research (grant agreement no. 8022-00187B) are further acknowledged. Quantum chemical calculations were performed on the Vienna Scientific Cluster (Austria). The authors also acknowledge Xiaosong Xue (Nankai University) and Aneta Turlik (University of California, Los Angeles) for fruitful discussions. 


\section{References}

[1] N. K. Devaraj, ACS Central Science 2018, 4, 952-959.

[2] H. C. Hang, C. Yu, D. L. Kato and C. R. Bertozzi, Proceedings of the National Academy of Sciences 2003, 100, 14846.

[3] M. L. W. J. Smeenk, J. Agramunt and K. M. Bonger, Current Opinion in Chemical Biology 2021, 60, 79-88.

[4] S. S. Nguyen and J. A. Prescher, Nature Reviews Chemistry 2020, 4, 476-489.

[5] a) M. L. Blackman, M. Royzen and J. M. Fox, Journal of the American Chemical Society 2008, 130, 13518-13519; b) B. L. Oliveira, Z. Guo and G. J. L. Bernardes, Chemical Society Reviews 2017, 46, 4895-4950.

[6] E. J. L. Stéen, P. E. Edem, K. Nørregaard, J. T. Jørgensen, V. Shalgunov, A. Kjaer and M. M. Herth, Biomaterials 2018, 179, 209-245.

[7] a) R. Rossin, P. Renart Verkerk, S. M. van den Bosch, R. C. M. Vulders, I. Verel, J. Lub and M. S. Robillard, Angewandte Chemie International Edition 2010, 49, 3375-3378; b) A. H. A. M. van Onzen, R. M. Versteegen, F. J. M. Hoeben, I. A. W. Filot, R. Rossin, T. Zhu, J. Wu, P. J. Hudson, H. M. Janssen, W. ten Hoeve and M. S. Robillard, Journal of the American Chemical Society 2020, 142, 10955-10963; c) B. M. Zeglis, C. Brand, D. Abdel-Atti, K. E. Carnazza, B. E. Cook, S. Carlin, T. Reiner and J. S. Lewis, Molecular Pharmaceutics 2015, 12, 3575-3587; d) M. Charton, Steric Effects in Drug Design (Berlin, Heidelberg) 1983, pp. 107-118; e) E. J. L. Stéen, J. T. Jørgensen, C. Denk, U. M. Battisti, K. Nørregaard, P. E. Edem, K. Bratteby, V. Shalgunov, M. Wilkovitsch, D. Svatunek, C. B. M. Poulie, L. Hvass, M. Simón, T. Wanek, R. Rossin, M. Robillard, J. L. Kristensen, H. Mikula, A. Kjaer and M. M. Herth, ACS Pharmacology \& Translational Science 2021, 4, 824-833.

[8] a) Z. Li, H. Cai, M. Hassink, M. L. Blackman, R. C. D. Brown, P. S. Conti and J. M. Fox, Chemical communications (Cambridge, England) 2010, 46, 8043-8045; b) J. Sečkutè and N. K. Devaraj, Current opinion in chemical biology 2013, 17, 761-767; c) J. C. Knight, S. Richter, M. Wuest, J. D. Way and F. Wuest, Organic \& Biomolecular Chemistry 2013, 11, 3817-3825.

[9] a) J.-P. Meyer, J. L. Houghton, P. Kozlowski, D. Abdel-Atti, T. Reiner, N. V. K. Pillarsetty, W. W. Scholz, B. M. Zeglis and J. S. Lewis, Bioconjugate chemistry 2016, 27, 298-301; b) J. Zhu, S. Li, C. Wängler, B. Wängler, R. B. Lennox and R. Schirrmacher, Chemical Communications 2015, 51, 12415-12418; c) C. Denk, D. Svatunek, T. Filip, T. Wanek, D. Lumpi, J. Fröhlich, C. Kuntner and H. Mikula, Angewandte Chemie International Edition 2014, 53, 9655-9659; d) O. Keinänen, X.-G. Li, N. K. Chenna, D. Lumen, J. Ott, C. F. M. Molthoff, M. Sarparanta, K. Helariutta, T. Vuorinen and A. D. Windhorst, ACS medicinal chemistry letters 2016, 7, 62-66; e) Z. Li, H. Cai, M. Hassink, M. L. Blackman, R. C. D. Brown, P. S. Conti and J. M. Fox, Chemical Communications 2010, 46, 
8043-8045; f) N. K. Devaraj, G. M. Thurber, E. J. Keliher, B. Marinelli and R. Weissleder, Proceedings of the National Academy of Sciences 2012, 109, 4762-4767.

[10] R. García-Vázquez, U. M. Battisti, J. T. Jørgensen, V. Shalgunov, L. Hvass, D. L. Stares, I. N. Petersen, F. Crestey, A. Löffler, D. Svatunek, J. L. Kristensen, H. Mikula, A. Kjaer and M. M. Herth, Chemical Science 2021, 12, 11668-11675.

[11] a) F. Liu, Y. Liang and K. N. Houk, Journal of the American Chemical Society 2014, 136, $11483-$ 11493; b) S. Kronister, D. Svatunek, C. Denk and H. Mikula, Synlett 2018, 29, 1297-1302.

[12] T. G. Le, A. Kundu, A. Ghoshal, N. H. Nguyen, S. Preston, Y. Jiao, B. Ruan, L. Xue, F. Huang, J. Keiser, A. Hofmann, B. C. H. Chang, J. Garcia-Bustos, A. Jabbar, T. N. C. Wells, M. J. Palmer, R. B. Gasser and J. B. Baell, Journal of Medicinal Chemistry 2018, 61, 10875-10894.

[13] A. Stumpf, Z. K. Cheng, D. Beaudry, R. Angelaud and F. Gosselin, Organic Process Research \& Development 2019, 23, 1829-1840.

[14] T. Drapier, P. Geubelle, C. Bouckaert, L. Nielsen, S. Laulumaa, E. Goffin, S. Dilly, P. Francotte, J. Hanson, L. Pochet, J. S. Kastrup and B. Pirotte, Journal of Medicinal Chemistry 2018, 61, 52795291.

[15] Y. Qu, F.-X. Sauvage, G. Clavier, F. Miomandre and P. Audebert, Angewandte Chemie International Edition 2018, 57, 12057-12061.

[16] U. M. Battisti, K. Bratteby, J. T. Jørgensen, L. Hvass, V. Shalgunov, H. Mikula, A. Kjær and M. M. Herth, Journal of Medicinal Chemistry 2021.

[17] D. Svatunek, C. Denk, V. Rosecker, B. Sohr, C. Hametner, G. Allmaier, J. Fröhlich and H. Mikula, Monatshefte für Chemie - Chemical Monthly 2016, 147, 579-585.

[18] a) C. Denk, D. Svatunek, T. Filip, T. Wanek, D. Lumpi, J. Fröhlich, C. Kuntner and H. Mikula, Angew Chem Int Ed Engl 2014, 53, 9655-9659; b) S. Otto and J. B. F. N. Engberts, Pure and Applied Chemistry 2000, 72, 1365-1372; c) Z. Yang, C. Doubleday and K. N. Houk, Journal of Chemical Theory and Computation 2015, 11, 5606-5612.

[19] M. D. Liptak, K. C. Gross, P. G. Seybold, S. Feldgus and G. C. Shields, Journal of the American Chemical Society 2002, 124, 6421-6427.

[20] C. Hansch, A. Leo and R. W. Taft, Chemical Reviews 1991, 91, 165-195.

[21] D. Svatunek, M. Wilkovitsch, L. Hartmann, K. Houk and H. Mikula in Uncovering the key role of distortion in tetrazine ligations guides the design of bioorthogonal tools with high reactivity and superior stability, Vol. Chemistry, 2021.

[22] R. M. Versteegen, R. Rossin, W. ten Hoeve, H. M. Janssen and M. S. Robillard, Angewandte Chemie International Edition 2013, 52, 14112-14116.

[23] J.-D. Chai and M. Head-Gordon, Physical Chemistry Chemical Physics 2008, 10, 6615-6620. 
[24] B. P. Pritchard, D. Altarawy, B. Didier, T. D. Gibson and T. L. Windus, Journal of Chemical Information and Modeling 2019, 59, 4814-4820.

[25] P. Pracht, F. Bohle and S. Grimme, Physical Chemistry Chemical Physics 2020, 22, 7169-7192. [26] in bobbypaton/GoodVibes: GoodVibes v3.0.0, Vol. Zenodo, 2019. 


\section{Can we predict the substitution influence on the reaction kinetic?}

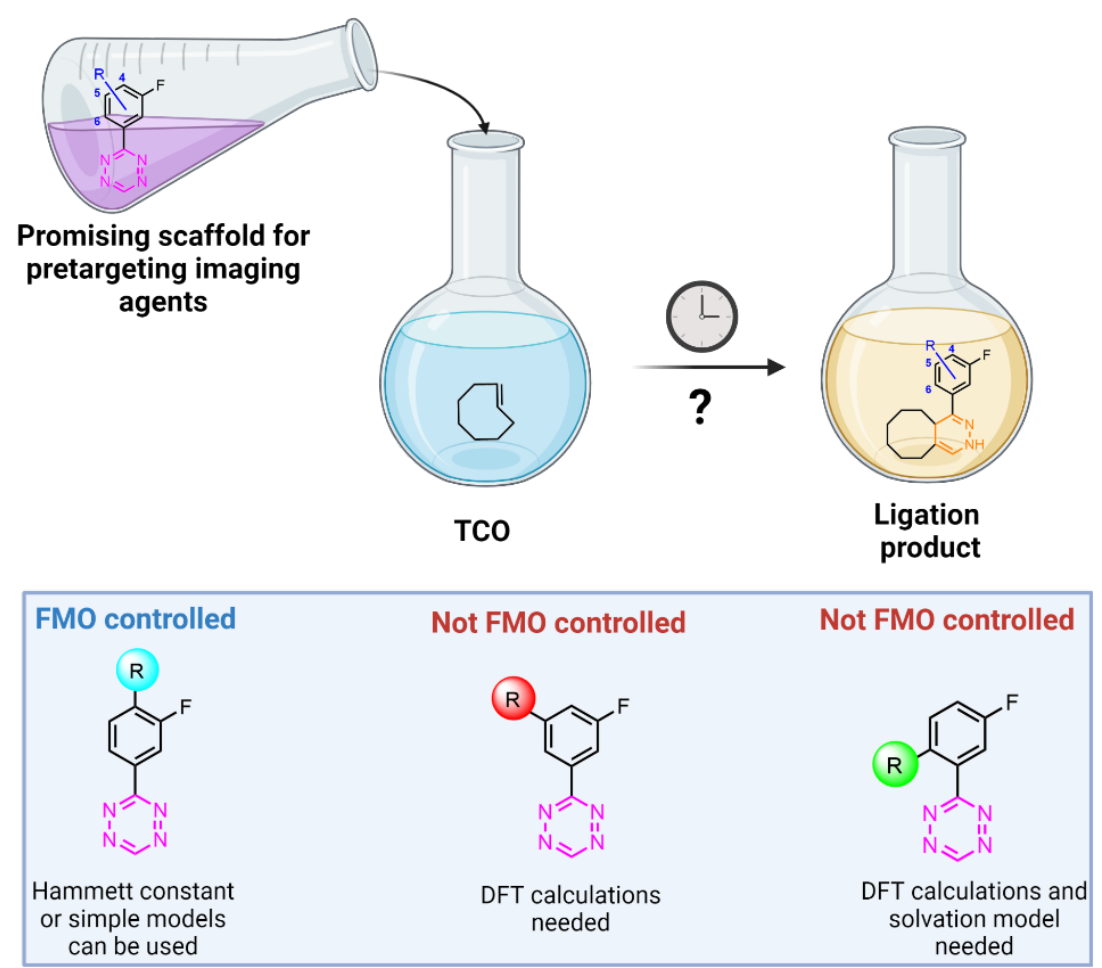

Tuning the tetrazine ligation: 3-(3-fluorophenyl)-1,2,4,5-tetrazine proved to be an interesting scaffold for the development of PET pretargeting agents. Here, 40 different tetrazine analogues have been synthesized and fully characterized. Their reaction kinetics vs trans-cyclooctene have been evaluated. These data were used to find correlations that can be exploited to predict the reactivity of new tetrazines. 